\title{
The POLAR gamma-ray burst polarization catalog
}

\author{
M. Kole ${ }^{1}$, N. De Angelis ${ }^{1}$, F. Berlato ${ }^{2}$, J. M. Burgess 2 , N. Gauvin ${ }^{3}$, J. Greiner ${ }^{2}$, W. Hajdas ${ }^{4}$, H. C. Li ${ }^{5,6}$, Z. H. Li $i^{5,6}$, \\ A. Pollo ${ }^{7,8}$, N. Produit ${ }^{3}$, D. Rybka ${ }^{7}$, L. M. Song ${ }^{5,6}$, J. C. Sun ${ }^{5,6}$, J. Szabelski ${ }^{7}$, T. Tymieniecka ${ }^{7}$, Y. H. Wang ${ }^{5,6}$, \\ B. B. $\mathrm{Wu}^{5}, \mathrm{X} . \mathrm{Wu}^{1}$, S. L. Xiong ${ }^{5}$, S. N. Zhang ${ }^{5,6}$, and Y. J. Zhang ${ }^{5}$
}

\author{
${ }^{1}$ Department of Nuclear and Particle Physics, University of Geneva, 24 quai Ernest-Ansermet, 1205 Geneva, Switzerland \\ e-mail: merlin.kole@unige.ch \\ 2 Max-Planck-Institut fur Extraterrestrische Physik, Giessenbachstrasse 1, 85748 Garching, Germany \\ 3 University of Geneva, Geneva Observatory, ISDC, 16, Chemin d'Ecogia, 1290 Versoix, Switzerland \\ ${ }^{4}$ Paul Scherrer Institut, 5232 Villigen, Switzerland \\ 5 Key Laboratory of Particle Astrophysics, Institute of High Energy Physics, Chinese Academy of Sciences, Beijing 100049, \\ PR China \\ ${ }^{6}$ University of Chinese Academy of Sciences, Beijing 100049, PR China \\ 7 National Centre for Nuclear Research, ul. A. Soltana 7, 05-400 Otwock, Swierk, Poland \\ 8 Astronomical Observatory of the Jagiellonian University, ul. Orla 171, 30-244 Cracow, Poland
}

Received 9 March 2020 / Accepted 14 September 2020

\section{ABSTRACT}

\begin{abstract}
Context. Despite over 50 years of research, many open questions remain about the origin and nature of gamma-ray bursts (GRBs). Linear polarization measurements of the prompt emission of these extreme phenomena have long been thought to be key to answering a range of these questions. The POLAR detector was designed to produce the first set of detailed and reliable linear polarization measurements in the 50-500 keV energy range. During late 2016 and early 2017, POLAR detected a total of 55 GRBs. The analysis results of 5 of these GRBs have been reported, and were found to be consistent with a low or unpolarized flux. However, previous reports by other collaborations found high levels of linear polarization, including some as high as $90 \%$.

Aims. We study the linear polarization for the 14 GRBs observed by POLAR for which statistically robust inferences are possible. Additionally, time-resolved polarization studies are performed on GRBs with sufficient apparent flux.

Methods. A publicly available polarization analysis tool, developed within the Multi-Mission Maximum Likelihood framework (3ML), was used to produce statistically robust results. The method allows spectral and polarimetric data from POLAR to be combined with spectral data from the Fermi Gamma-ray Burst Monitor (Fermi-GBM) and the Neil Gehrels Swift Observatory to jointly model the spectral and polarimetric parameters.

Results. The time-integrated analysis finds all results to be compatible with low or zero polarization with the caveat that, when timeresolved analysis is possible within individual pulses, we observe moderate linear polarization with a rapidly changing polarization angle. Therefore, time-integrated polarization results, while pointing to lower polarization, are potentially an artifact of summing over the changing polarization signal and thus washing out the true moderate polarization. We therefore caution against overinterpretation of any time-integrated results inferred herein and encourage the community to wait for more detailed polarization measurements from forthcoming missions such as POLAR-2 and LEAP.
\end{abstract}

Key words. polarization - methods: data analysis - instrumentation: polarimeters - catalogs - gamma-ray burst: general

\section{Introduction}

Gamma-ray bursts (GRBs) are the brightest electromagnetic phenomena in the Universe since the Big Bang. They consist of initial bright bursts of $\mathrm{X}$ - and gamma-rays, known as the prompt phase, which last from hundreds of milliseconds up to hours. The prompt emission is followed by a longer lasting afterglow which has been observed from radio wavelengths up to $\mathrm{TeV}$ energies. Historically GRBs have been divided into two categories (Mazets et al. 1981), namely short GRBs, which have a prompt phase lasting less than $2 \mathrm{~s}$, and long GRBs, which have a prompt phase of over 2 s. In 2017, a GRB was shown to originate from the merger of two neutron stars thanks to the detection of a gravitational wave counterpart to GRB 170817A (Abbott 2019). As a result of detailed localization measurements with optical instruments, strong evidence exists that long GRBs are connected to the collapse of massive stars (Woosley 1993). The spectral and timing properties for both short and long GRBs suggest that the gamma-ray component of the prompt emission is produced in highly relativistic jets (Gehrels \& Razzaque 2013). However, the physical properties of these jets, such as their structure and magnetic topology, remain poorly understood despite the plethora of flux, spectral, and timing measurements produced over the last five decades; see for example von Kienlin et al. (2020), Lien et al. (2016), Meegan (1997) and Tsvetkova et al. (2017a).

The two remaining key parameters of the gamma-ray emission, the polarization degree (PD) and angle (PA), remain largely unprobed, despite popular belief that precise measurements of these parameters will provide unique information on the nature of the highly relativistic jets in which the prompt emission is produced (Toma et al. 2009). In particular, the linear polarization properties of the gamma-rays are highly dependent on the emission processes at play during the prompt emission as well as the magnetic fields and their structure within the emitting jets. For a detailed overview the reader is referred to, for example, Toma et al. (2009) and Gill et al. (2020). The theoretical predictions can be generalized as follows: Models predicting the 
majority of the emission to be the result of synchrotron radiation allow for linear PDs as high as 50\% (Luytikov et al. 2003). For emission coming from regions with a highly ordered magnetic field, the average linear PD will be $40 \%$ within a sample of GRBs (Toma et al. 2009). In dissipative photospheric models, the predicted linear PD is relatively low, on the order of a few percent, although it can be as high as $40 \%$ when the jet is seen at large off-axis angles (Lundman et al. 2016). Additionally, a high linear PD (around 50\%) is possible in such models as well at lower energies (typically below $10 \mathrm{keV}$ ), while at higher energies it is close to $0 \%$ (Lundman et al. 2016). Finally, the Internal-Collision-Induced Magnetic Reconnection and Turbulence (ICMART) models predict a decaying linear PD during each pulse of the prompt emission. In these models, the maximum value at the beginning of the pulse can be as high as $60 \%$, while the minimum value decreases to a few percent as presented in Zhang \& Yan (2011). In order to properly distinguish between such models, both a large sample of GRB measurements and measurements capable of determining the evolution of the linear polarization parameters within a single emission pulse are required (Gill et al. 2020). It should be noted that circular polarization is not predicted in any of these models (Luytikov et al. 2003; Lundman et al. 2014; Zhang \& Yan 2011), and therefore for the remainder of this work the term polarization can be assumed to mean linear polarization.

The large resolving power of the polarization parameters has prompted many attempts to perform detailed measurements in the past. However, the results often suffer from large statistical uncertainties as a result of the low efficiency of gammaray polarimeters, which is typically an order of magnitude less than that of spectrometers. Many of these measurements have additionally been shown to suffer from problems with systematic errors or mistakes in the analysis (McConnell 2017). Overall, the existing measurements do now show a coherent result for the PD of the prompt emission. An overview can be found in (Covino \& Götz 2016). Although some of the reported measurements correspond to low PDs, the majority of the published results concern measurements of high PDs. There are nevertheless several reasons for this bias towards larger PDs. The first is that at least several measurements where the PD was found to be compatible with an unpolarized flux were not published. This is for example the result for at least four GRBs measured by the GAP collaboration (Yonetoku 2013). A second and perhaps more important reason is that systematic errors in polarization measurements will almost always result in an over-estimation of the PD. This is a result of both the positive definite nature of the PD and the way polarization is measured. The latter reason for bias toward larger PDs is explored in greater detail in the following section. A significant number of detailed measurements from a dedicated gamma-ray polarimeter are required to allow for a more clear view of the polarization parameters of the prompt emission of GRBs. The POLAR mission, which underwent detailed calibration measurements both on ground (Kole et al. 2017) and in orbit (Li et al. 2018), thereby reducing systematic errors significantly, measured a total of 55 GRBs during the mission lifetime. Using these data here, we aim to provide the first catalog of detailed polarization measurements capable of resolving some of the open questions regarding GRBs.

\section{The POLAR mission}

POLAR was a dedicated GRB polarimeter launched as part of the Chinese Tiangong-2 (TG-2) Space Lab on September 15, 2016. The instrument, of which a detailed description can be

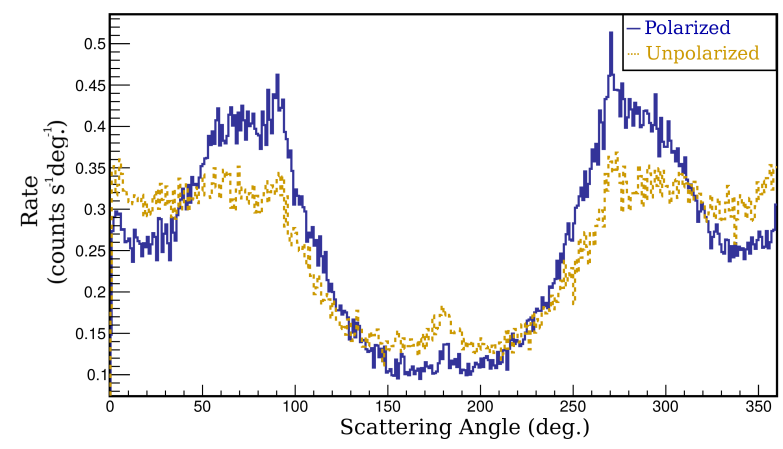

Fig. 1. An example of a simulated scattering angle distribution of polarized (blue) and unpolarized (brown dotted) flux as would be produced by POLAR for an off-axis GRB (170207A is taken as an example here). Both distributions show a $360^{\circ}$ modulation induced by the offaxis incoming angle of the GRB as well as some instrument-induced effects. The polarized distribution additionally shows a $180^{\circ}$ modulation, as well as an additional small $360^{\circ}$ modulation induced by the polarization of the incoming flux.

found in Produit et al. (2018), made use of a segmented scintillator array of $40 \times 40$ plastic bars, optimized for performing polarization measurements in the 50-500 keV energy range. The plastic scintillators were readout in groups of 64 using multianode photomutiplier tubes (MAPMTs). The polarization of an incoming gamma-ray flux can be derived from measurements of the scattering angle distribution of the incoming photons. This scattering angle is measured by POLAR using the scintillator array. Useful polarization events consist of photons that scatter at least once in one of the scintillators and subsequently (within a 100 ns time window) interact, either through Compton scattering or through photo-absorption, in a second scintillator. The scattering angle is then deduced from the position of the two scintillators in which an energy deposition was measured. Finally, a distribution of the scattering angles, often referred to as a modulation curve in the polarimetry community, can be produced for a full measurement. An example of such distributions, simulated using the POLAR Monte Carlo software (Kole et al. 2017) for $100 \%$ polarized and for $100 \%$ unpolarized flux can be seen in Fig. 1.

Using a perfect instrument, the scattering angle distribution from a polarized flux would show only a $180^{\circ}$ modulation, while that from an unpolarized flux would be flat. For actual instruments the scattering angle distributions appear significantly more complex; first, because GRBs typically enter the instrument with a nonzero incoming angle with respect to zenith (the off-axis angle). A $360^{\circ}$ modulation is induced as photons scattering back towards the incoming direction of the GRB have a greater chance of escaping the instrument. Second, instrumental effects are seen in the distribution. For POLAR one clear effect is the presence of peaks at $90^{\circ}$ intervals induced by the square geometry of the instrument, which favors some scattering angles over others. Other, less clearly visible effects stem from nonuniformity in detector sensitivity, for example, as a result of differences in efficiency for different MAPMT channels. The $180^{\circ}$ modulation induced by the polarization is added to all these other effects in case of a polarized flux. In addition to a $180^{\circ}$ modulation, the polarization can induce a second $360^{\circ}$ degree modulation especially for GRBs with a large off-axis incoming angle. It should therefore be noted that the frequently used method of dividing the measured scattering angle distribution by a simulated distribution of an unpolarized flux, which removes 
all effects not induced by polarization from the distribution, and subsequently fitting this with a $180^{\circ}$ modulation is therefore not correct. This is especially true for instruments with a wide field of view.

It should furthermore be stressed that instrumental effects can also induce a $180^{\circ}$ modulation, which, if not modeled correctly in the $\mathrm{MC}$, will appear as a polarization signal in the analysis. It is therefore crucial for polarimeters to be well calibrated such that all features in the scattering angle distribution are understood and well modeled in the instrument response. Any discrepancies between the real and modeled instrument response can, in the analysis, result in a false polarization signal, whereas they are unlikely to result in an underestimation of the polarization. This results from the effect that polarization adds fluctuations to the scattering angle distribution, which is flat for an unpolarized flux; while model-induced errors are highly likely to induce fluctuations that make the distribution less flat, it is unlikely for such errors to flatten a distribution. To reduce systematic effects, the POLAR instrument was carefully calibrated both on ground, of which a detailed report can be found in Kole et al. (2017), and subsequently in orbit (Li et al. 2018; Xiao et al. 2018). As reported by Kole et al. (2017), the polarization measured for an unpolarized flux, which is induced due to uncertainties in the instrument response as well as due to statistical uncertainties, was of the order of $2 \%$. This error is taken as a systematic error induced by uncertainties in the instrument response in all the analyses presented in this work. The error is directly taken into account for all the results presented here by adding it to the simulated scattering angle distributions.

In-orbit calibration of the polarization response of POLAR to verify the systematic error is not possible due to the current lack of standard-candle-like sources for gamma-ray polarization. The source closest to being a standard candle is the Crab as several measurements exist that largely agree, as detailed for example by Chauvin et al. (2017) and references therein. As POLAR has a large field of view, polarization measurements of the nebula are not possible as it is a continuous source. Measurements of the pulsar are possible, albeit challenging, using time-resolved analysis. The Crab pulsar was previously used to calibrate the timing precision of POLAR ( $\mathrm{Li}$ et al. 2017) and its energy response (Li et al. 2019). Analysis of the polarization is currently ongoing (Li et al., in prep.). Although the preliminary results agree with previous measurements, such as those by Chauvin et al. (2017), the precision of the POLAR measurements is not sufficient to use this as a calibration source because of the significant difficulty in such measurements with a wide-field-of-view instrument. Although the careful in-orbit calibration has significantly improved our understanding of the detector response, and therefore likely the systematic error, it is currently not possible to quantify this improvement due to a lack of an in-orbit calibration source. The systematic error of $2 \%$ is therefore kept despite likely being an over-estimation of this error.

\section{Analysis}

As the analysis method used in this catalog differs from that conventionally used in polarization analyses, as well as that used previously by the POLAR collaboration, we provide some details of the analysis method here. It should be noted that the method used here was previously used to produce the timeresolved analysis results for GRB 170114A detected by POLAR (Burgess et al. 2019). While here we give an overview of the analysis and a justification for using it, the reader is referred to Burgess et al. (2019) for details.

\subsection{Original POLAR data analysis}

The first polarization results from GRBs detected by POLAR were presented by Zhang et al. (2019a). The analysis method applied there, which was used to extract the polarization parameters of five GRBs, consists of the following steps:

- Selecting a signal and background interval for the GRB. The background interval is taken to be significantly longer than the GRB in order to minimize statistical errors. Additionally, special care is taken for the background to not contain any lowenergy afterglow or other forms of contamination.

- The data from both intervals are processed using the data pipeline described in Li et al. (2018) in order to select polarization events and to calculate the scattering angles of these events.

- The scattering angle distributions of the background is subtracted from that of the signal region while taking into account the difference in live time of the two intervals.

- A set of 61 simulations for the particular GRB is performed using the MC software described in Kole et al. (2017). The simulations are performed using an energy spectrum provided by other instruments, such as Fermi Gamma-ray Burst Monitor (Fermi-GBM) or Konus-Wind, and a location of the GRB, again provided by other instruments. The simulations are performed for both unpolarized flux and for $100 \%$ polarized fluxes with 60 different PAs. Additionally, the temperatures as measured throughout the POLAR detector, which slightly modify the electronics behavior ( $\mathrm{Li}$ et al. 2018), are taken into account when producing the response.

- The 61 simulated results are processed using the same analysis pipeline used for the measurement data.

- Scattering angle distributions are produced for 101 different PDs and 60 different PAs (giving a total of 6060 different parameter configurations) by combining the scattering angle distributions of the 61 simulations at the correct ratio.

- The scattering angle distribution of the measured signal is directly fitted to all the 6060 simulated distributions. The $\chi^{2}$ values of the 6060 fits are used to produce a $\Delta \chi^{2}$ distribution in PD and PA space. Finally, the polarization of the measured flux along with confidence intervals are deduced from this distribution.

A very similar method was previously used for the analysis of the GAP data (Yonetoku et al. 2011). However, several improvements to this analysis method are possible. Firstly, the background subtraction method, although frequently used for such types of analysis, is not the proper way of statistically handling data with Poisson errors. As a result of this procedure, statistical information is lost and the error propagation is not accurate. As described in more detail in Burgess et al. (2019) a better way to correct for the background is by fitting the background and subsequently taking it into account by modeling it in the analysis. This method ensures that the statistics are handled correctly while also producing a more accurate estimation of the background and its fluctuations during the signal region.

A second issue is that the simulations are performed for one specific spectrum. The first problem with this is that in order to study the effects of a different input spectrum on the polarization measurement, the simulation process has to be fully repeated. Second, errors on the spectral parameters used are not naturally carried over into the polarization results. A range of simulations was performed by Zhang et al. (2019a) in order to propagate the spectral errors to the polarization parameters. In these simulations, the spectral parameters were varied within their uncertainty followed by the full reanalysis of the polarization for each new spectrum. This process was repeated 10000 times. The 
distribution of the polarization parameters produced with these simulations was then used to estimate the systematic error. As this process was highly time consuming it was performed for only one GRB and the errors were assumed to be representative for all five GRBs studied in that work. Although this method is satisfactory, it is clearly not the optimum method to take the spectral uncertainties into account.

Finally, the method fully relies on spectral information which is acquired independently from the polarization analysis. For example, when using spectral data from Fermi-GBM, the spectral fit results as provided by that collaboration in their Gammaray Coordination Network notices (GCNs) are used as fixed input parameters in the analysis. The polarization analysis is then performed fully independently. The spectral information from POLAR remains fully unused despite the possibility for this data to improve the spectral analysis and thereby to reduce systematic errors in the polarization analysis.

\subsection{Improved analysis using 3ML}

An analysis method which tackles all the above issues was developed and implemented for the first time in the time-resolved analysis of GRB 170114A described in Burgess et al. (2019). There, instead of performing simulations with a fixed spectrum, the polarization responses for 150 discrete energy ranges (from 5 to $755 \mathrm{keV}$ in $5 \mathrm{keV}$ steps) were simulated separately. By subsequently adding the produced responses in the correct ratio, the polarization response for each possible spectrum can be produced by simply folding the spectrum through this response. While the initial simulation is time consuming, it allows us to study the effect of the spectral parameters on the results of the polarization analysis without the need for starting simulations for a specific new spectrum. More importantly, the spectral response of POLAR was simulated, allowing the polarization parameters to be fitted at the same time as the spectrum using POLAR data only. This makes it possible to analyse GRBs which were only observed by POLAR.

The whole of the analysis detailed above can be performed directly using the 3ML framework (Vianello et al. 2015) which contains both spectral analysis and polarization analysis tools. Although the latter was designed for POLAR specifically, it was designed to be easily adapted to work with data from any other gamma-ray polarimeter. An additional advantage of using the 3ML framework is that it allows to joint fits to be performed using data from different instruments. Therefore the spectral information of POLAR can be combined with that of FermiGBM, for example, in order to perform a joint fit of the spectrum (using POLAR and Fermi-GBM data) and the polarization (using POLAR data only). This method therefore allows us to not only study GRBs that were previously not analyzable due to the lack of spectral information, but also to obtain more precise measurements thanks to joint fitting of the spectrum and the polarization. The background is furthermore fitted and subsequently modeled, thereby improving both the precision and allowing for a proper error propagation.

A schematic overview of the analysis procedure can be seen in Fig. 2. First, from the light curve, a signal and background period are selected based on $T_{90}$ calculations. Typically one second is added before the start time of the $T_{90}$ period and an equal period is added towards the end to ensure that the full emission episode is captured. This period was often corrected by eye when needed in case of low emission at the start of the GRB for example, in which case it is clarified in the upcoming analysis section of the specific GRB. For the background selection, periods both before and after the GRB are selected while excluding potential late emission or other features in the light curve. It should be noted that previous extensive studies presented by Zhang et al. (2019a) show that the background region selection has no significant influence on the polarization results.

Based on the selection, both a spectrum (in count space) and scattering angle distribution (also in count space) can be produced for the signal and the background region. The instrument response for the spectrum, which has been produced for one specific incoming angle of the GRB, can subsequently be used, with spectral parameters picked from a prior distribution, to produce a range of modeled spectra (also in count space). The modeled and the measured spectra can then be used to calculate the likelihood. In parallel, the same spectral parameters are, together with polarization parameters (also picked from their respective flat prior distributions), used to produce the scattering angle distributions corresponding to the specific spectral shape described by the spectral parameters. Each of these modeled scattering angle distributions is therefore for one specific set of spectral parameters and polarization parameters, and can be used together with the measured distribution to calculate the likelihood. The method differs here from all previous analyses where the spectral fit is performed first followed by a polarization analysis using the results from the spectral analysis as input. Here the two are performed in parallel.

It is worth noting here that even though the number of bins is often reduced for illustrative purposes when presenting the scattering angle distribution in upcoming figures, the likelihood for the scattering angle distribution is calculated using distributions with 360 bins for each GRB. A joint likelihood can then be calculated for the set of spectral and polarization parameters which is subsequently minimized. The final posterior parameter distributions are obtained using MultiNest (version PyMultiNest 2.9; Feroz et al. 2009; Buchner et al. 2014). It should finally be noted that, as opposed to the analyses presented by for example Zhang et al. (2019a), Yonetoku et al. (2011), and Chattopadhyay et al. (2019), the measured and modeled scattering angle distributions are not normalized to ensure an equal number of total counts in both. Rather, the number of events per scattering bin is in units of counts $\mathrm{s}^{-1}$, both in the measured and modeled distributions. The value used here was used in order to ensure that potential issues with the effective area become apparent in the analysis. Such issues are ignored in the traditional analysis through the normalization process.

\subsection{Catalog analysis}

The production of the simulation responses, as well as the Bayesian analysis used for joint fitting the spectral and polarization parameters, is described in detail in Burgess et al. (2019). Almost the same analysis procedure is followed for the work presented here. However, it should be noted that in Burgess et al. (2019) the spectral fits were performed using a synchrotron model. For the production of the catalog presented here, the spectral fits are performed using the Band function (Band et al. 1993) or a cutoff powerlaw (CPL). The Band function is an exponentially cutoff power law smoothly connected to a power law at high energy. The CPL is defined here as $F(E)=$ $K(E / 100 \mathrm{keV})^{- \text {index }} e^{-E / E_{\mathrm{c}}}$. Here $K$ (counts s $\left.{ }^{-1} \mathrm{~cm}^{-2} \mathrm{keV}^{-1}\right)$ is the normalization constant, $E$ is the energy in $\mathrm{keV}$, index is the powerlaw index, and $E_{\mathrm{c}}$ is the cutoff energy.

The decision to use empirical models, such as the Band function and CPL, was taken to make the results independent of the physical model used. Both models have ubiquitous use as 


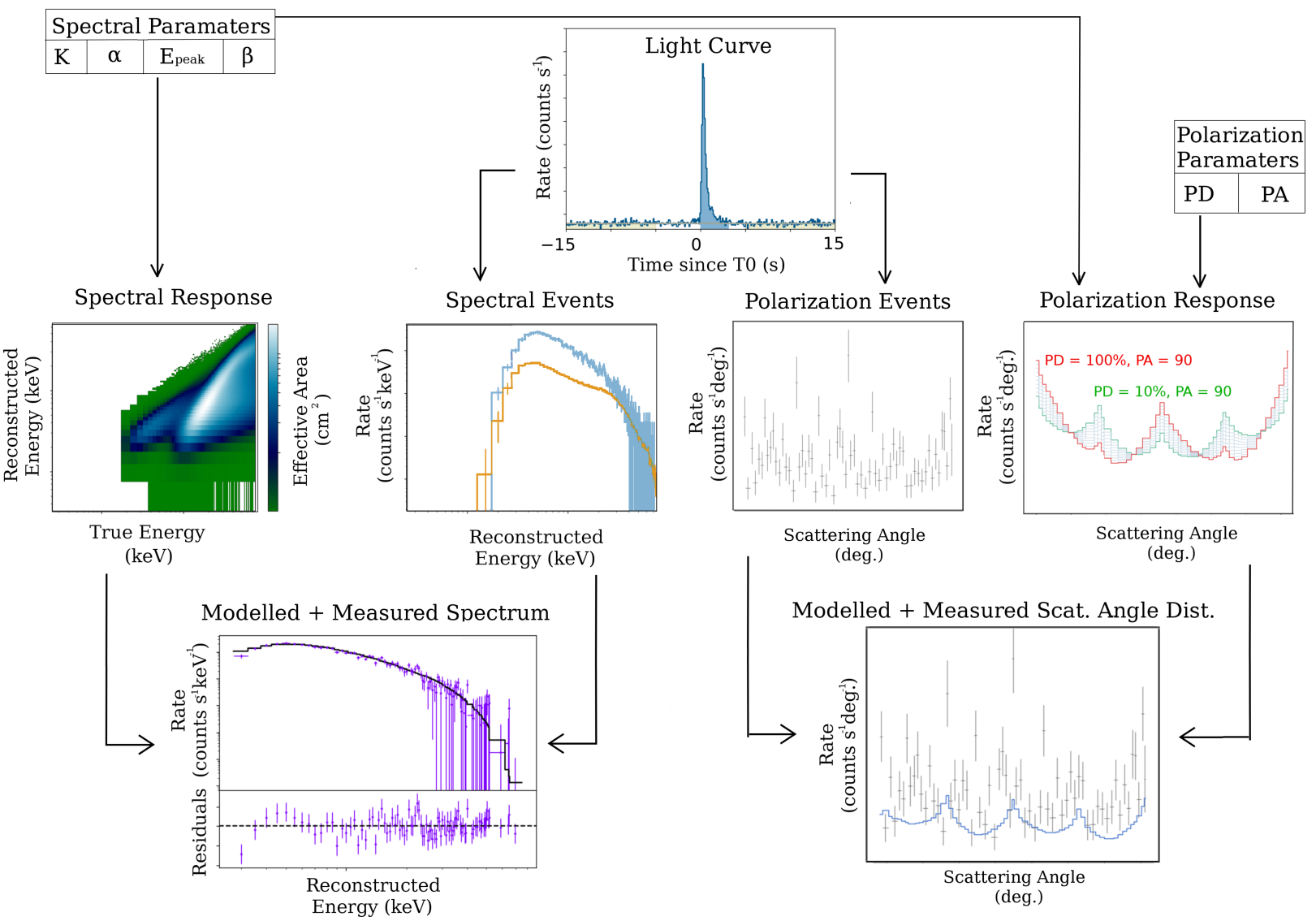

Fig. 2. Schematic representation of the analysis method used here. In step one, both the spectral and polarization events are extracted from the data. These are used to produce a spectrum (in count space) and a scattering angle distribution. Spectral parameters are picked from the provided priors and combined with the spectral response to produce a spectrum (again in count space) which is used together with the measured spectrum to calculate a likelihood. In parallel, these spectral parameters are, together with polarization parameters from a prior, provided to the polarization response in order to produce a scattering angle distribution. This is then used to calculate a likelihood using the measured distribution. Going through this process for different parameters in order to minimize the likelihood, a posterior distribution is produced for both the spectral and polarization parameters.

a phenomenological fitting function for GRB spectra as they describe the shape of most GRBs adequately. However, recent studies show that the Band function is a poor model for the physical emission spectra (e.g., Burgess et al. 2015; Burgess 2019; Zhang et al. 2019b) and should only be used as a qualitative description of the spectra such as the peak energy and flux. However, Yassine et al. (2020) also show that the Band function may not adequately model the true peak energy either. The use of physical models to perform the analysis, as in Burgess et al. (2019), would be preferable to avoid over-interpretation of the spectral parameters found using empirical models. However, in order to allow for a model-independent interpretation of the results presented here, the use of empirical models was chosen.

The selection to use either a Band function or a CPL in the final analysis is based on an initial analysis where only the spectrum of each GRB was analyzed. The use of either of these two empirical models in the final analysis was based on the deviance information criterion (Spiegelhalter 2002) found in this initial analysis.

As we use the Band function or CPL for spectral fitting, we perform a simultaneous fit for each GRB using the spectral parameters $\alpha, E_{\text {peak }}$, and $\beta$ for Band and index and $E_{\mathrm{c}}$ for CPL. The parameter $K$, which serves as a normalisation constant for both functions, is also fitted along with the two polarization parameters PD and PA. In cases where spectral data are available from either Fermi-GBM or the Swift-Burst Alert Telescope (BAT), such data are used in the analysis. In cases where data from Fermi-GBM are used, the data from at least three of its $\mathrm{NaI}$ sub-detectors are used in the analysis as well as one of the BGO detectors. These four detectors are selected based on the significance of the detection of the GRB as calculated using the standard tools available in $3 \mathrm{ML}^{1}$. The three NaI detectors with the highest significance are selected along with the BGO detector with the highest significance. In case data from more than one mission are used in the spectral analysis, additional normalization parameters are fitted to allow for the correction of the effective area of either of the instruments. This method is typically applied by the Fermi collaboration to correct for unknown inter-calibration issues between its different detectors (Ackermann et al. 2013). In all the analyses performed in this work, the corrections are found to be compatible to within $25 \%$ of 1.0 when using POLAR and Fermi-GBM data, and below $10 \%$ when using POLAR and Swift-BAT. Such values are similar to those found, for example, in catalog analysis of FermiGBM for inter-calibration detection for the Fermi-Large Area Telescope (LAT) and the different NaI (corrections of the order of $5 \%$ ) and BGO detectors (corrections of the order of $25 \%$; Ackermann et al. 2013). Here we therefore find that POLAR,

1 https://threeml . readthedocs.io 
Swift-BAT, and Fermi-GBM are well calibrated with respect to one another. Additionally, no obvious systematic issues in the spectral fits were found for any of the GRBs analyzed here, indicating that the POLAR instrument response is well modeled. This includes GRBs with large off-axis incoming angles (those exceeding the limit set by Zhang et al. 2019a of $45^{\circ}$ ). The analysis of GRBs with large off-axis incoming angles was not performed in previous POLAR publications as there was concern that material in the vicinity of POLAR, such as the surface of Tiangong- 2 or its solar panels, would not be modeled correctly. The concern was that incorrectly modeled materials would induce systematic errors in the response. It was previously not possible to properly asses the existence of such systematic errors, whereas this is possible now using the joint spectral fits with other well-established spectrometers. As we do not find obvious systematic issues for off-axis GRBs, we can relatively safely assume the polarization response produced using the same MC software to also be accurate. Finally it should be clarified that the analysis procedure for time-resolved analyses presented here for certain GRBs is identical to that employed for the analysis of the full GRB. As joint fits are performed for the spectrum and polarization, spectral evolution during the GRB, which could otherwise induce significant systematic errors in the polarization results, is automatically taken into account using the method employed here.

After launch in September 2016, POLAR detected a total of at least 55 GRBs (Xiong et al. 2017). Operation of POLAR ended in April 2017 after the instrument experienced a problem with the high voltage power supply. It should be noted that all 55 GRBs reported in Xiong et al. (2017) have been detected by other instruments. Additional GRBs, not detected by other instruments, could be present in the POLAR data, but no significant candidates have been found in preliminary studies. We therefore only use the 55 GRBs reported by Xiong et al. (2017) in this study. An initial spectral analysis (without fitting the polarization parameters) was performed using 3ML on all GRBs detected while POLAR was taking data in its science mode (data were taken in calibration mode as well for several weeks) and for which the incoming angle of the GRB was found to be $90^{\circ}$ or smaller. The spectral results for all these GRBs, of which there are 38 in total, are presented in Table 1 along with the $T_{90}$ for all 38 , which was calculated using the POLAR data. The $T_{90}$ was calculated based on counts in the 10 to $750 \mathrm{keV}$ energy range. Table 1 additionally contains a fit result for the late emission of GRB $170127 \mathrm{C}$ using both POLAR and Fermi-GBM data, as well as a fit result for a specific time period of GRB 170131A. This latter information was added as this is the only GRB observed by all three instruments, meaning POLAR, Fermi-GBM, and Swift-BAT, although Swift-BAT data are not available for the full GRB. Despite a lack of data for the full GRB, a fit of a specific time interval was still performed as this allowed us to study the inter-calibration of the three instruments together for the first time. The analysis found effective area corrections below $10 \%$ for all instruments, indicating that the three instruments are well calibrated against one another.

For the results presented previously in Zhang et al. (2019a) the selection criteria to perform polarization analysis on a GRB were as follows:

- The GRB has been observed by detectors other than POLAR and measurements of both the spectrum and location are provided by other instruments.

- The fluence of the GRB, as provided by other instruments in the $10-1000 \mathrm{keV}$ energy range, exceeds $5 \times 10^{-6} \mathrm{erg} \mathrm{cm}^{-2}$.
- The incoming angle with respect to the POLAR instrument zenith, $\theta$, is below $45^{\circ}$.

For the study here, the first criterion is dropped because no external spectrum is required to perform the analysis due to the fact that the spectrum and polarization are fitted at the same time. Although no external spectrum is required, relying on the POLAR data for the spectral fit will typically result in greater systematic error; however, this error is automatically propagated to the posterior distribution. In case Fermi-GBM or Swift-BAT data are available, they are used together with the POLAR data to perform a joint fit. For Konus-Wind observations, this is currently not possible as the data are not publicly available in a format compatible with the 3ML software. Therefore, in the analysis performed here the Konus-Wind spectral parameters, when available, are used as the priors for the spectral parameters in the fit.

For the third criterion listed above, the accepted off-axis angle is increased to $90^{\circ}$ in the selection procedure as no significant problems were found in the instrument response for large off-axis angles using joint spectral studies.

The main selection criterion remaining is therefore based on the fluence of the GRB. As such fluence measurements are not reported for all GRBs observed using the other instruments, fluence measurements based on the POLAR data are used instead. An average flux, as measured over a $1 \mathrm{~s}$ bin is used as the selection criterion here. The average flux was used here instead of the fluence as it is more representative for the signal-tobackground-counts ratio in the data. The lower limit was set to $1 \times 10^{-6} \mathrm{erg} \mathrm{cm}^{-2} \mathrm{~s}^{-1}$ measured in the $10-1000 \mathrm{keV}$ energy range.

Finally, the POLAR instrument response is simulated based on the best available localization measurement of the GRB. Typically, this is either a location from Swift, the localization provided by Fermi-LAT, or is calculated using InterPlanetary Network (IPN) triangulation. When none of these are available, the Fermi-GBM data are used for localization using the BAyesian Location Reconstruction Of GRBs (BALROG) method described by Berlato et al. (2019). If additionally no Fermi-GBM data are available, the location is calculated using POLAR data using the method described by Wang et al. (2020).

The uncertainty on the location will induce a systematic error in the polarization results. This effect was previously studied for the POLAR mission and is presented in Wang et al. (2020) and in Zhang et al. (2019a). The systematic error resulting from the location is taken into account here in the same way as was done by Zhang et al. (2019a). This method consists of adding the uncertainty to the polarization model by adding errors to the data points in the modeled scattering angle distribution. The size of these errors is based on the work performed by Zhang et al. (2019a), where it was found that the uncertainty on the data point in the model is approximately linear with the error in the uncertainty, where a $1 \sigma$ error of $1^{\circ}$ on the location results in a $1 \%$ uncertainty on the data points in the modeled scattering angle distribution. While this method results in systematic errors of approximately the correct magnitude, it does not allow us to properly take into account asymmetric errors on the location (such as those typically seen in IPN locations). For asymmetric location errors, the average location error with a single digit precision is used to obtain a good approximation of the systematic error. A method to overcome this shortcoming in our analysis is to perform joint fits for the spectrum, location, and polarization. Although this is theoretically possible, such a method would require very large CPU time and will require development and careful studies; this will be a future project of the authors. 
Table 1. Spectral properties of the 38 GRBs detected by POLAR with an off-axis angle below $90^{\circ}$ while the instrument was in science mode.

\begin{tabular}{|c|c|c|c|c|c|c|}
\hline GRB & Band or CPL & $T_{90}(\mathrm{~s})$ & Fluence $^{(*)}$ & $\alpha /$ index & $E_{\text {peak }} / E_{\mathrm{c}}(\mathrm{keV})$ & $\beta$ \\
\hline $161203 \mathrm{~A}$ & Band & $4.1 \pm 0.1$ & $\left(5.0_{-0.8}^{+0.7}\right) \times 10^{-6}$ & $0.13_{-0.25}^{+0.27}$ & $344_{-12}^{+19}$ & $-3.41_{-0.46}^{+0.39}$ \\
\hline $161205 \mathrm{~A}$ & Band & $15.8 \pm 3.4$ & $\left(1.4_{-0.1}^{+0.1}\right) \times 10^{-6}$ & $-1.39_{-0.09}^{+0.17}$ & $119_{-21}^{+28}$ & $-3.08_{-0.58}^{+0.48}$ \\
\hline $161207 \mathrm{~A}$ & Band & $33.3 \pm 1.4$ & $\left(6.1_{-0.2}^{+0.6}\right) \times 10^{-6}$ & $-0.44_{-0.13}^{+0.17}$ & $197_{-26}^{+29}$ & $-2.30_{-0.40}^{+0.27}$ \\
\hline 161207B & Band & $5.1 \pm 0.3$ & $\left(4.1_{-1.0}^{+2.2}\right) \times 10^{-7}$ & $-0.98_{-0.28}^{+0.36}$ & $1680_{-790}^{+1350}$ & $-3.17_{-0.50}^{+0.61}$ \\
\hline 161210A & CPL & $3.14 \pm 1.26$ & $\left(5.4_{-4.4}^{+7.6}\right) \times 10^{-7}$ & $-0.89_{-0.18}^{+0.18}$ & $830_{-310}^{+310}$ & NA \\
\hline $161212 \mathrm{~A}$ & CPL & $1.44 \pm 0.16$ & $\left(3.3_{-3.2}^{+4.3}\right) \times 10^{-7}$ & $-1.20_{-0.16}^{+0.16}$ & $\begin{array}{l}1040_{-290}^{+290} \\
\end{array}$ & NA \\
\hline 161217B & $\mathrm{CPL}$ & $2.66 \pm 0.2$ & $\left(1.7_{-0.9}^{+2.9}\right) \times 10^{-7}$ & $-1.32_{-0.15}^{+0.10}$ & $300_{-180}^{+650}$ & NA \\
\hline $161217 \mathrm{C}$ & Band & $6.33 \pm 0.25$ & $\left(9.0_{-1.9}^{+2.7}\right) \times 10^{-6}$ & $-1.08_{-0.25}^{+0.13}$ & $143_{-34}^{+32}$ & $-2.76_{-0.61}^{+0.36}$ \\
\hline $161218 \mathrm{~A}$ & Band & $11.48 \pm 0.14$ & $\left(2.0_{-0.2}^{+0.3}\right) \times 10^{-5}$ & $-0.54_{-0.06}^{+0.07}$ & $144_{-11}^{+12}$ & $-2.51_{-0.15}^{+0.14}$ \\
\hline $161228 \mathrm{~A}$ & Band & $67.4 \pm 2.58$ & $\left(3.8_{-07}^{+1.3}\right) \times 10^{-6}$ & $-0.96_{-0.19}^{+0.00}$ & $182_{-46}^{+56}$ & $-3.05_{-0.56}^{+0.15}$ \\
\hline 161228B & Band & $9.75 \pm 1.55$ & $\left(9.6_{-1.0}^{+1.6}\right) \times 10^{-7}$ & $-1.22_{-0.09}^{+0.12}$ & $171_{-27}^{+37}$ & $-2.98_{-0.60}^{+0.48}$ \\
\hline $161228 \mathrm{C}$ & Band & $21.76 \pm 1.52$ & $\left(3.4_{-0.4}^{+0.8}\right) \times 10^{-6}$ & $-0.57_{-0.13}^{+0.14}$ & $232_{-27}^{+30}$ & $-2.96_{-0.58}^{+0.53}$ \\
\hline 161229A & Band & $31.26 \pm 0.44$ & $\left(3.1_{-0.1}^{+0.2}\right) \times 10^{-5}$ & $-0.64_{-0.03}^{+0.03}$ & $339_{-14}^{+12}$ & $-3.07_{-1.49}^{+0.72}$ \\
\hline 161230A & CPL & $3.92 \pm 0.13$ & $\left(4.4_{-2.5}^{+6.0}\right) \times 10^{-7}$ & $-1.24_{-0.10}^{+0.12}$ & $\begin{array}{l}650_{-260}^{+360}\end{array}$ & NA \\
\hline $170101 \mathrm{~A}$ & CPL & $2.02 \pm 0.11$ & $\left(6.4_{-1.3}^{+1.6}\right) \times 10^{-6}$ & $-1.55_{-0.03}^{+0.06}$ & $323_{-30}^{+34}$ & NA \\
\hline 170101B & Band & $11.14 \pm 0.38$ & $\left(8.0_{-0.5}^{+0.7}\right) \times 10^{-6}$ & $-0.59_{-0.05}^{+0.05}$ & $232_{-10}^{+10}$ & $-3.28_{-0.49}^{+0.46}$ \\
\hline $170105 \mathrm{~A}$ & Band & $3.23 \pm 1.6$ & $\left(4.3_{-1.1}^{+0.6}\right) \times 10^{-6}$ & $-1.22_{-0.2}^{+0.03}$ & $50_{-13}^{+14}$ & $-3.16_{-0.43}^{+0.30}$ \\
\hline 170109A & Band & $258.4 \pm 1.7$ & $\left(8.9_{-0.4}^{+0.7}\right) \times 10^{-5}$ & $-0.88_{-0.04}^{+0.04}$ & $869_{-83}^{+108}$ & $-3.14_{-0.48}^{+0.41}$ \\
\hline $170114 \mathrm{~A}$ & Band & $10.48 \pm 0.16$ & $\left(2.0_{-0.2}^{+0.3}\right) \times 10^{-5}$ & $-0.68_{-0.09}^{+0.09}$ & $211_{-25}^{+31}$ & $-1.87_{-0.05}^{+0.04}$ \\
\hline $170120 \mathrm{~A}$ & Band & $8.55 \pm 2.72$ & $\left(8.7_{-1.0}^{-0.2}\right) \times 10^{-7}$ & $-0.19_{-0.22}^{+0.09}$ & $126_{-11}^{+14}$ & $-3.23_{-0.46}^{+0.037}$ \\
\hline $170121 \mathrm{~A}$ & Band & $6.12 \pm 0.19$ & $\left(3.3_{-0.4}^{+0.7}\right) \times 10^{-6}$ & $-0.32_{-0.12}^{+0.15}$ & $567_{-60}^{+57}$ & $-1.88_{-0.24}^{+0.18}$ \\
\hline $170127 \mathrm{C}$ & CPL & $0.14 \pm 0.01$ & $\left(3.4_{-1.4}^{+5.3}\right) \times 10^{-6}$ & $0.25_{-0.11}^{+0.12}$ & $358_{-28}^{+31}$ & $\mathrm{NA}$ \\
\hline $170127 \mathrm{C}^{(* *)}$ & Band & NA & $\left(5.8_{-1.1}^{+0.8}\right) \times 10^{-6}$ & $-1.14_{-0.21}^{+0.22}$ & $1500_{-900}^{+800}$ & $-3.1_{-0.6}^{+0.6}$ \\
\hline 170130A & Band & $31.08 \pm 0.69$ & $\left(3.1_{-0.5}^{+0.8}\right) \times 10^{-6}$ & $-0.94_{-0.14}^{+0.16}$ & $202_{-41}^{+43}$ & $-2.58_{-0.76}^{+0.40}$ \\
\hline $170131 \mathrm{~A}$ & Band & $30.4 \pm 9.4$ & $\left(4.6_{-0.4}^{+0.6}\right) \times 10^{-6}$ & $-1.39_{-0.07}^{+0.07}$ & $158_{-22}^{+29}$ & $-3.05_{-0.55}^{+0.48}$ \\
\hline $170131 \mathrm{~A}^{(* * *)}$ & Band & NA & NA & $-1.28_{-0.08}^{+0.07}$ & $196_{-30}^{+41}$ & $-3.06_{-0.59}^{+0.45}$ \\
\hline $170206 \mathrm{~A}$ & Band & $1.26 \pm 0.01$ & $\left(6.5_{-03}^{+0.4}\right) \times 10^{-6}$ & $-0.49_{-0.03}^{+0.08}$ & $344_{-12}^{+13}$ & $-2.68_{-0.19}^{+0.14}$ \\
\hline $170206 \mathrm{C}$ & $\mathrm{CPL}$ & $22.48 \pm 0.31$ & $\left(1.4_{-0.8}^{+1.3}\right) \times 10^{-5}$ & $-1.8_{-0.1}^{+0.1}$ & $880_{-300}^{+300}$ & $\mathrm{NA}$ \\
\hline $170207 \mathrm{~A}$ & Band & $38.76 \pm 0.26$ & $\left(6.7_{-0.2}^{+0.2}\right) \times 10^{-5}$ & $-0.87_{-0.02}^{+0.02}$ & $475_{-20}^{+19}$ & $-3.37_{-1.26}^{+0.74}$ \\
\hline $170208 \mathrm{C}$ & Band & $45.93 \pm 0.23$ & $\left(1.4_{-0.2}^{+0.3}\right) \times 10^{-5}$ & $-0.75_{-0.11}^{+0.11}$ & $176_{-21}^{+25}$ & $-2.01_{-0.16}^{+0.09}$ \\
\hline $170210 \mathrm{~A}$ & Band & $47.63 \pm 2.51$ & $\left(6.6_{-0 .}^{+0.3}\right) \times 10^{-5}$ & $-0.96_{-0.02}^{+0.02}$ & $462_{-22}^{+22}$ & $-2.72_{-0.49}^{+0.10}$ \\
\hline 170219A & Band & $0.09 \pm 0.02$ & $\left(3.9_{-0.8}^{+1.9}\right) \times 10^{-7}$ & $-0.40_{-0.16}^{+0.20}$ & $510_{-110}^{+150}$ & $-2.10_{-0.44}^{+0.29}$ \\
\hline $170228 B$ & CPL & $26.99 \pm 0.78$ & $\left(4.9_{-1.7}^{+1.9}\right) \times 10^{-6}$ & $-1.36_{-0.05}^{+0.10}$ & $347_{-54}^{+63}$ & NA \\
\hline $170305 \mathrm{~A}$ & Band & $0.45 \pm 0.01$ & $\left(1.6_{-0.2}^{+0.2}\right) \times 10^{-6}$ & $-0.35_{-0.09}^{+0.09}$ & $253_{-16}^{+17}$ & $-3.20_{-0.49}^{+0.41}$ \\
\hline 170306B & Band & $19.88 \pm 0.22$ & $\left(1.3_{-0.1}^{+0.2}\right) \times 10^{-5}$ & $-0.62_{-0.04}^{+0.04}$ & $273_{-12}^{+10}$ & $-2.96_{-0.49}^{+0.43}$ \\
\hline $170309 \mathrm{~A}$ & Band & $2.03 \pm 0.03$ & $\left(2.9_{-0.2}^{+0.5}\right) \times 10^{-6}$ & $-1.42_{-0.03}^{+0.04}$ & $303_{-90}^{+126}$ & $-3.02_{-0.60}^{+0.64}$ \\
\hline $170315 \mathrm{~A}$ & Band & $22.70 \pm 5.68$ & $\left(1.1_{-0.2}^{+0.2}\right) \times 10^{-6}$ & $-1.06_{-0.20}^{+0.24}$ & $52.8_{-5.8}^{+6.3}$ & $-3.28_{-0.45}^{+0.44}$ \\
\hline 170317A & Band & $13.74 \pm 0.82$ & $\left(3.2_{-1.3}^{+2.2}\right) \times 10^{-6}$ & $-1.21_{-0.22}^{+0.40}$ & $60_{-29}^{+39}$ & $-2.78_{-0.51}^{+0.34}$ \\
\hline $170320 \mathrm{~A}$ & Band & $6.83 \pm 0.09$ & $\left(6.7_{-0.6}^{+0.8}\right) \times 10^{-6}$ & $-0.24_{-0.17}^{+0.13}$ & $228_{-15}^{+13}$ & $-2.32_{-0.21}^{+0.16}$ \\
\hline $170325 B$ & Band & $7.82 \pm 0.76$ & $\left(3.5_{-0.5}^{+0.5}\right) \times 10^{-6}$ & $-0.62_{-0.36}^{+0.37}$ & $125_{-12}^{+13}$ & $-3.41_{-0.42}^{+0.41}$ \\
\hline
\end{tabular}

Notes. The $T_{90}$ parameter is additionally presented $\left({ }^{(*)}\right.$ in units of $\mathrm{erg} \mathrm{cm}^{-2}$ in $10-1000 \mathrm{keV}$ based on $T_{90}$ period), ${ }^{(* *)}$ Afterglow time interval only (from $T 0+2.0 \mathrm{~s}$ to $20.0 \mathrm{~s}$ ), ${ }^{(* *)} \mathrm{GRB}$ fitted using only the time interval where Swift-BAT has data (from $T 0+16.6 \mathrm{~s}$ to $26.5 \mathrm{~s}$ ).

Finally, the above criteria would select a total of 15 GRBs. However, GRB 161129A is dropped from the analysis as it occurred on the tail of a large solar flare (RHESSI Collaboration 2020); as a result, potential systematic errors from a quickly changing background could exist, making the measurement unreliable. In total, 14 different $\mathrm{GRBs}^{2}$ are therefore discussed here. Of these, two are clear single pulse GRBs with a Fast

\footnotetext{
2 The data products used for the analysis of these 14 GRBs will be placed on https://www.astro.unige.ch/polar/ and the collaboration strongly encourages additional analysis by external groups.
}

Rise Exponential Decay (FRED) nature, 170101A and 170114A, while the rest consist of several overlapping pulses. Below we present the details for the analysis of GRB 170101A, a single pulse GRB where Swift-BAT data were used in the analysis to produce more constraining measurements than those previously published by Zhang et al. (2019a) for this GRB. Additionally, we present the analysis of $170207 \mathrm{~A}$, a bright GRB previously not considered in the analysis because of its large off-axis angle. Details of the analysis of the remaining 12 GRBs can be found in the Appendices of this work, while a summary of all the results can be found in Sect. 5 . 


\section{Analysis of GRBs}

\section{1. $170101 \mathrm{~A}$}

The GRB 170101A was detected by POLAR and by Swift-BAT. The latter defines $T 0$ as January 1, 2017 at 02:26:00.679 (UT) which is also used as $T 0$ in the analysis presented here for convenience. A $T_{90}$ of $(2.02 \pm 0.11) \mathrm{s}$ was measured using POLAR data for this GRB. Figure 3a shows the light curve, including the signal region (blue) and part of the background region (yellow). Spectral and polarization analysis was performed using both POLAR and Swift-BAT data. The response of POLAR was produced using the refined location provided by Swift-BAT: $\operatorname{RA}(\mathrm{J} 2000)=267.089^{\circ}, \operatorname{Dec}(\mathrm{J} 2000)=11.642^{\circ}($ Barthelmy et al. 2017). The spectral results of the fit can be seen in Fig. 3. The effective area correction (applied to the POLAR data) found in the analysis is $1.07 \pm 0.04$. The posterior distributions of the spectral and polarization parameters are shown in Fig. 4. The posterior distribution of the polarization parameters is shown in Fig. 5 together with the measured scattering angle distribution, with the posterior model predictions (blue) superimposed on top. A PD of $6.3_{-6.3}^{+10.8 \%}$ (errors correspond to the $1 \sigma$ uncertainty bounds here and throughout the remainder of this paper unless otherwise specified) is found. This is compatible with that reported by Zhang et al. (2019a), but it should be noted that the PA used by Zhang et al. (2019a) is measured in the coordinate system as defined by the International Astronomical Union (IAU); see di Serego Alighieri (2017) for details. Here, on the other hand, we present the PA in the POLAR coordinate system. The PA as measured following the IAU convention is presented in Fig. 14 towards the end of this paper. A $99 \%$ credibility upper limit of $35.1 \%$ is found.

As GRB 170101A has a sufficiently high signal-tobackground-counts ratio to perform basic time-resolved analysis, this GRB was divided into two time bins with similar statistics. The first time bin ranged from $T=0.0 \mathrm{~s}$ to $T=0.5 \mathrm{~s}$, and the second from $T=0.5 \mathrm{~s}$ to $T=2.0 \mathrm{~s}$. The polarization parameter posteriors for these time bins can be seen in Fig. 6 . The results are consistent with an unpolarized flux for both time bins within $99 \%$ credibility. However, for the first and shorter time bin, a PD of $(32 \pm 12) \%$ is found which suggests polarization. For the longer time bin, a PD of $13_{-13}^{+18} \%$ is found, consistent with a PD of $0 \%$. The results, with a PD of $32 \%$ in the first time bin and a potentially smeared out polarization signal in the second, longer time bin suggest that with a finer time binning a significant polarization with an evolving polarization angle could be found. The signal-to-background-count ratio in the POLAR data from this GRB does not allow for such an analysis.

\section{2. $170207 A$}

The GRB 170207A, a long GRB with three clearly separated active periods, each of which consists of overlapping pulses, was detected by both POLAR and Fermi-GBM. The latter reported a T0 of 2017-02-07 at 21:45:03.67 (UT) (Roberts et al. 2017), which is taken as $T 0$ for the analysis presented here for convenience. A $T_{90}$ of $(38.76 \pm 0.26) \mathrm{s}$ was measured using POLAR data. The light curve, including the signal region (blue) and part of the background region (yellow), can be seen in Fig. 7. The GRB was detected by Fermi-GBM (Roberts et al. 2017). The data from four of its detectors were therefore used for a combined fit with the POLAR data. The results of the spectral fit can be seen in Fig. 7. The effective area correction (applied to the POLAR data) found in the analysis was $0.94 \pm$ 0.02 . The polarization response of POLAR was produced using

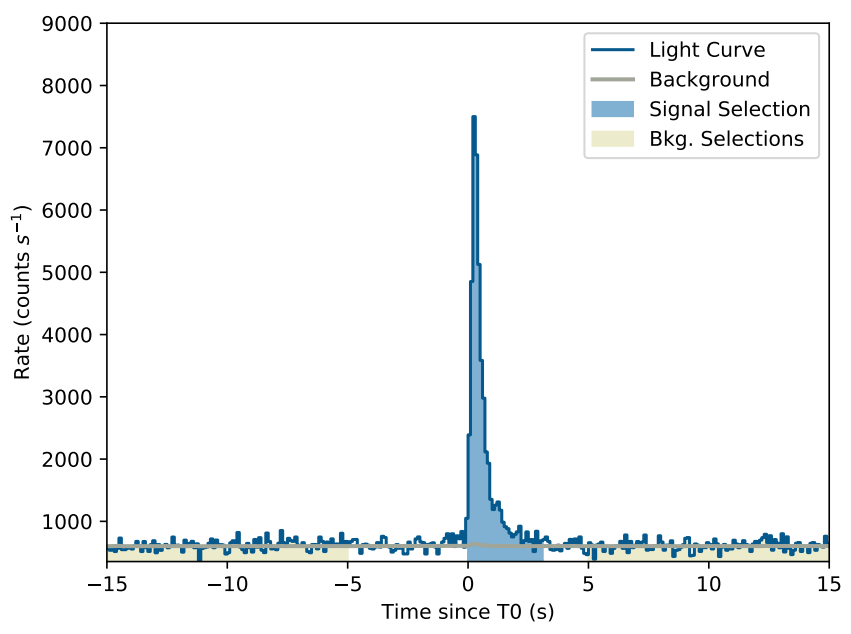

(a)

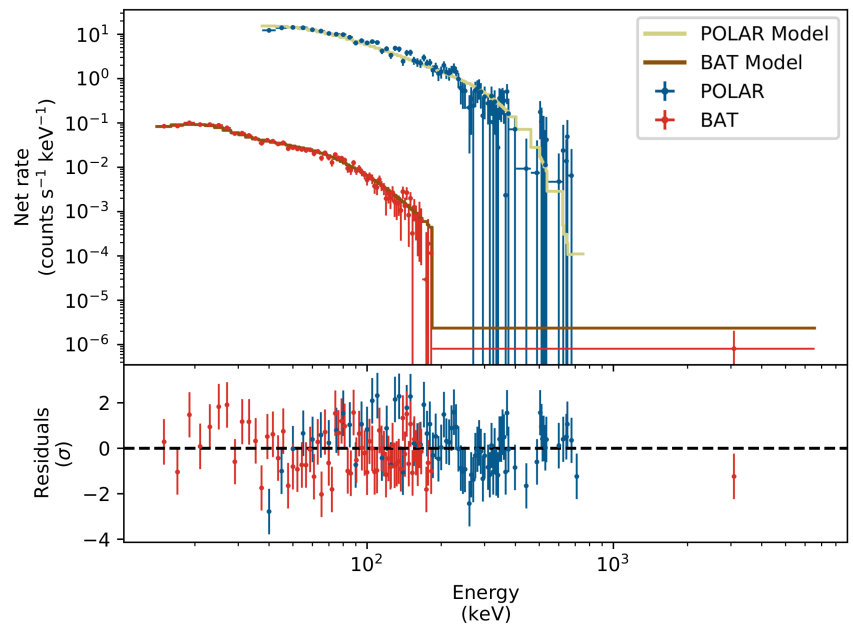

(b)

Fig. 3. Panel $a$ : light curve of GRB 170101A as measured by POLAR where $T=0 \mathrm{~s}$ is defined as the $T 0$ defined by $S w i f t$-BAT for this GRB. Panel $b$ : joint spectral fit result for 170101A. The number of counts as detected by both POLAR (blue) and Swift-BAT (red) are shown along with the best fitting spectrum folded through the instrument responses in yellow for POLAR data and in brown for Swift-BAT data. The residuals for both data sets are shown at the bottom of the figure.

the location calculated using the BALROG method detailed in Berlato et al. (2019) using Fermi-GBM data for this GRB, namely RA $(J 2000)=316.9^{\circ}$, Dec $(J 2000)=59.1^{\circ}$, and a localization error of $2^{\circ}$ was assumed in the response. The location of this GRB implies a significant off-axis incoming angle for the GRB of $67.2^{\circ}$.

As a result of the large off-axis incoming angle of the GRB, the sensitivity of POLAR is reduced for specific PAs. This is a result of the design of POLAR, which uses scintillator bars to measure the interaction locations of the photons. As there is no information on the interaction location within a bar, but only a 2D location, the precision of the bar cross section of $6 \times 6 \mathrm{~mm}^{2}$ inside of the detector is provided for each interaction. For GRBs that occur on-axis, this $2 \mathrm{D}$ plane is perpendicular to the incoming direction of the photons and therefore all polarization angles can be measured with the same precision. For GRBs that enter POLAR from the side, this is no longer the case. The effect is illustrated in Fig. 8 which shows the $M_{100}$ as a function of the 

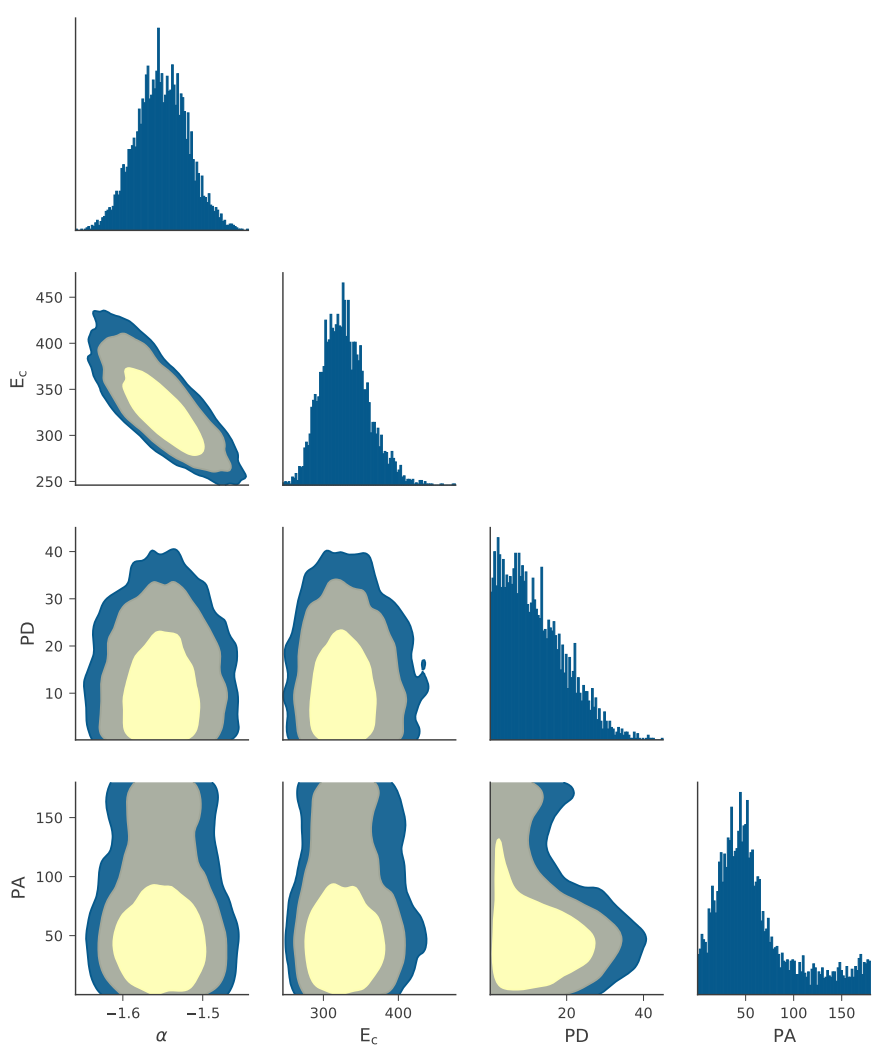

Fig. 4. Spectral and polarization posterior distributions for GRB 170101A. The $1 \sigma$ and $2 \sigma$ credibility intervals and that corresponding to $99 \%$ are indicated. The PA shown here is in the POLAR coordinate system; a rotation in the positive direction of 56 degrees transforms this to the coordinate system as defined by the IAU.

polarization angle for a GRB entering POLAR at $85^{\circ}$ off-axis. $M_{100}$ is a figure of merit which is defined as the amplitude of the $180^{\circ}$ modulation for a $100 \%$ polarized incoming flux. It is generally acquired by dividing a scattering angle distribution produced using a $100 \%$ polarized beam by the simulated scattering angle distribution for an unpolarized beam. The result is a scattering angle distribution which is a near perfect harmonic function with a $180^{\circ}$ modulation, although a smaller but significant $360^{\circ}$ modulation can also be found in this corrected scattering angle distribution. The amplitude of $180^{\circ}$ modulation is taken to be the $M_{100}$. It can be seen that for polarization angles of $45^{\circ}$ and $135^{\circ}$ (corresponding to polarization angles of $45^{\circ}$ degrees with respect to zenith direction) the sensitivity is almost zero. As all the plots in this paper give the PA as measured in the POLAR coordinate system, these minima are in the same location for all the GRBs presented in this work. This effect only becomes significant for off-axis incoming angles greater than approximately $65^{\circ}$.

The posterior distributions of the spectral and polarization parameters are shown in Fig. 9. Finally, the posterior distribution of the polarization parameters is shown together with measured scattering angle distribution superimposed by the posterior model predictions (blue) in Fig. 10. The scattering angle distribution clearly indicates the large off-axis angle resulting in the $360^{\circ}$ modulation in the distribution. A PD of $5.9_{-4.8}^{+9.6 \%}$ was found along with $99 \%$ credibility upper limit for PD of $35.9 \%$.

GRB 170207A has a sufficiently high signal-to-backgroundcounts ratio to perform basic time-resolved analysis. The three separate emission episodes were therefore analyzed individually.
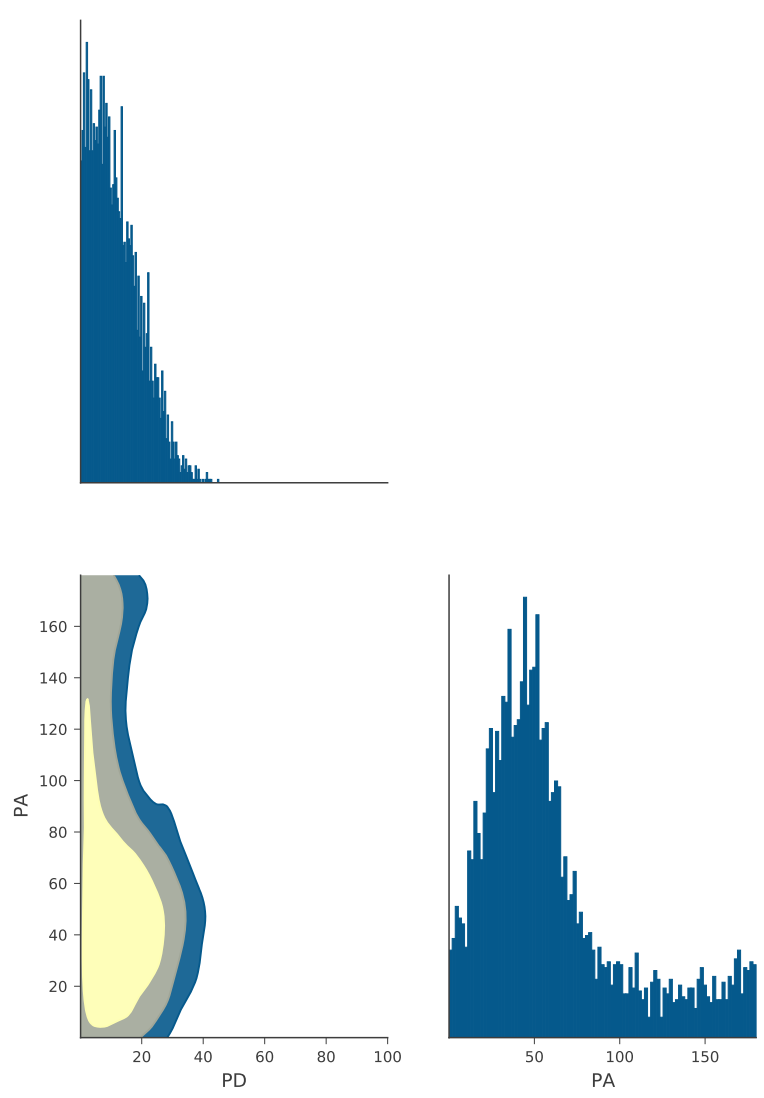

(a)

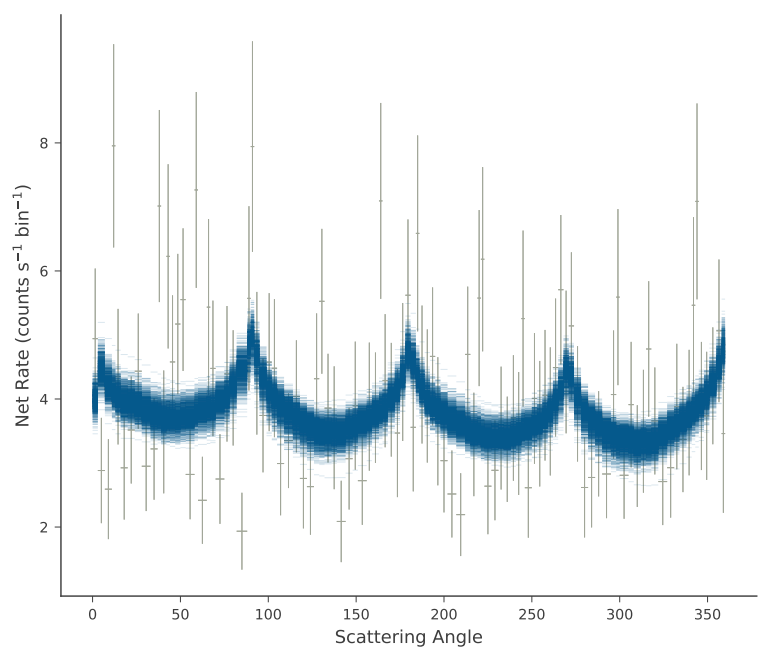

(b)

Fig. 5. Panel $a$ : polarization posterior distributions for GRB 170101A with the $1 \sigma$ and $2 \sigma$ credibility intervals as well as that corresponding to $99 \%$. The PA shown here is in the POLAR coordinate system; a rotation in the positive direction of 56 degrees transforms this to the coordinate system as defined by the IAU. Panel $b$ : measured scattering angle distribution (gray data points with 3 degree bin size) onto which the posterior model predictions (blue) are superimposed. The errors on the data points are the Poisson errors corrected for the background.

While constraining measurements were possible for the first two periods, this was not the case for the last period because of a lack of signal counts. Therefore, only the first two periods are discussed here. The first time bin ranged from $T=0.0$ to $10.0 \mathrm{~s}$, and the second from 15.0 to $25.0 \mathrm{~s}$. The polarization parameter 

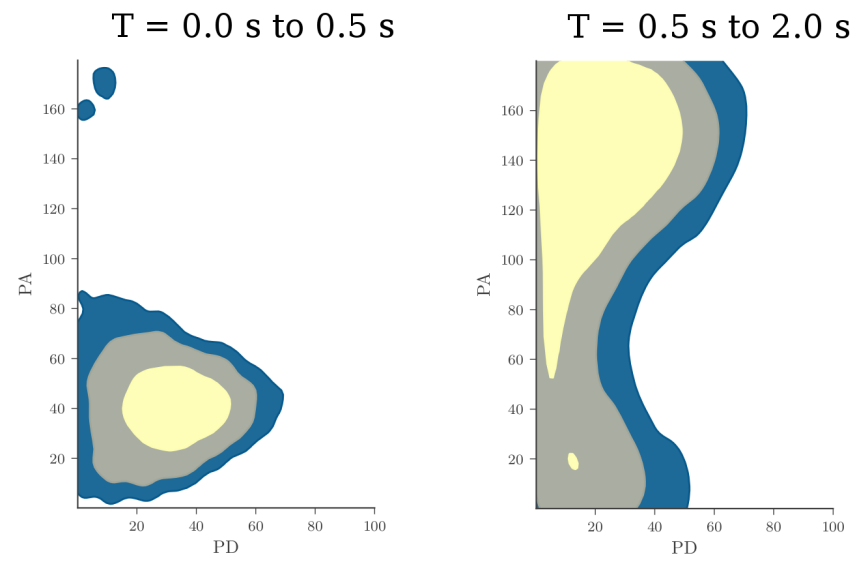

Fig. 6. Polarization posterior distributions for the two time bins of GRB $170101 \mathrm{~A}$ with the $1 \sigma$ and $2 \sigma$ credibility intervals as well as that corresponding to $99 \%$ credibility. On the left, the posteriors for the first time bin range from $T=0.0 \mathrm{~s}$ to $T=0.5 \mathrm{~s}$, while on the right for the second time bin these range from $T=0.5 \mathrm{~s}$ to $T=2.0 \mathrm{~s}$. The polarization angle shown here is in the POLAR coordinate system; a rotation in the positive direction of 56 degrees transforms this to the coordinate system as defined by the IAU.

posteriors can be seen in Fig. 11. The results indicate PDs of $9.1_{-7.8}^{+16.3 \%}$ and $7.4_{-6.1}^{+14.3} \%$ for time intervals 1 and 2 , respectively, and are therefore consistent with an unpolarized flux. Upper limits with $99 \%$ credibility for PD of $53.1 \%$ and $50.9 \%$ are found for these two time intervals. It should be noted here that despite being clearly separate time emission episodes within the GRB, both episodes consist of several overlapping pulses.

\section{Conclusion}

A more detailed analysis method, as previously used in Burgess et al. (2019), was employed to analyze the polarization of 14 GRBs detected by POLAR. Figure 12 shows a summary of the posteriors of the PDs for all 14 GRBs, while Fig. 13 presents a simplified illustration of the PDs together with the $1 \sigma$ error bars. The corresponding PAs are shown in Fig. 14 where the angles are defined following the IAU convention. Finally, the spectral and polarization parameters (including the fluence) found in this analysis for all 14 GRBs are summarized in Table 2. First, we can conclude that the new results are compatible with previously published polarization results of POLAR for 5 of the sample of 14 GRBs detected by POLAR (Zhang et al. 2019a; Burgess et al. 2019). We typically find low levels of polarization with the exception of GRB 170101B for which a PD of $60 \%$ is found. However, this is within a credibility of $90 \%$ to come from an unpolarized flux. The combination of the 14 posteriors shown in Fig. 12 is compatible with what one would expect for unpolarized or mostly unpolarized emission, despite the high PD found for GRB 170101B. While for several GRBs no tight constraints on the PD are possible because of a lack of sensitivity for specific PAs, it remains possible, for these GRBs, to exclude high PDs for all polarization angles with the exception of those for which there is poor sensitivity. This is specifically the case for GRBs 170210A and 161218A and to a lesser extent for GRBs 161203A, 161229A, and 170320A.

Besides an agreement for the time integrated polarization results with a low or unpolarized GRB flux, we again find strong evidence for intra-pulse evolution of the PA in singlepulse GRBs. The emission within single pulses appears to be polarized at around $30 \%$. However, the PD is washed out

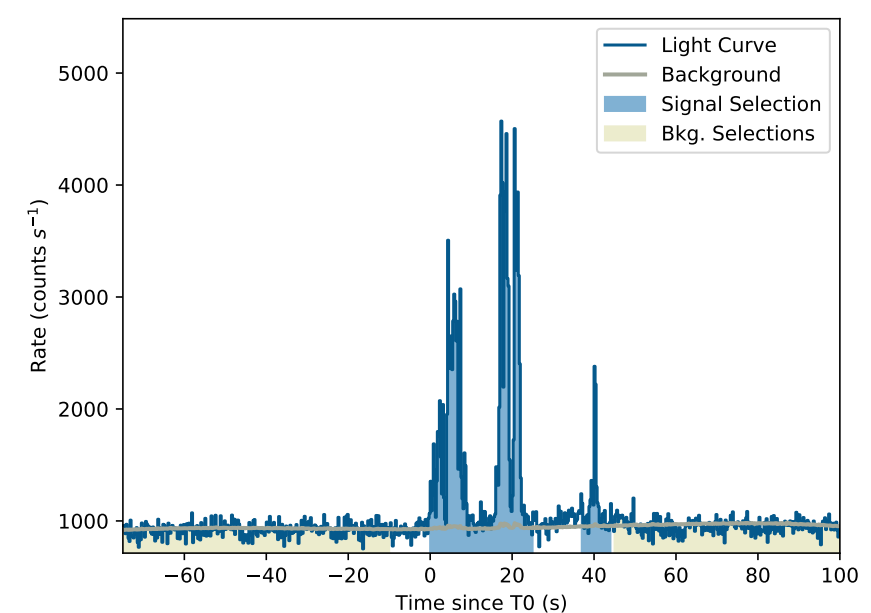

(a)

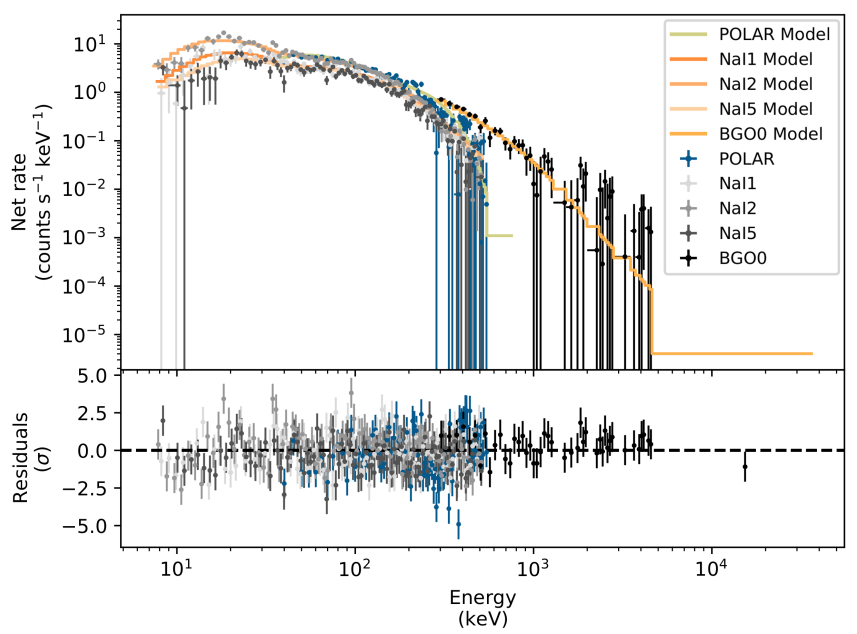

(b)

Fig. 7. Panel a: light curve of GRB 170207A as measured by POLAR, where $T=0 \mathrm{~s}$ is defined as the $T 0$ employed in the Fermi-GBM data products (Roberts et al. 2017). Panel b: joint spectral fit result for 170207A. The number of counts as detected by both POLAR (blue) and the different $\mathrm{NaI}$ and BGO detectors of Fermi-GBM (gray tints) are shown along with the best-fitting spectrum folded through the instrument responses in yellow for POLAR data and in orange tints for the Fermi-GBM data. The residuals for both data sets are shown at the bottom of the figure.

because of a quickly evolving PA. We confirm the strong hints previously presented for GRB 170114A by both Zhang et al. (2019a) and Burgess et al. (2019). Here we additionally find that GRB 170101A shows hints of this behavior. More precise measurements, such as those expected from POLAR-2 (Kole 2019), which is scheduled for launch in 2024, or LEAP (LEAP Collaboration 2016), currently proposed with a potential launch in 2025 , are needed to fully probe the time evolution of the polarization parameters. Finally, we only see signs of an evolving PA inside of single pulses. We do not see any signs of evolving polarization angles outside of individual pulses or in overlapping pulses, such as those reported for example by GAP for GRB 100826A (Yonetoku et al. 2011) or by the AstroSat CZT-Imager collaboration for GRB 160821A (which consists of a single emission episode but with several overlapping pulses) and 160325A (Sharma et al. 2019, 2020). 


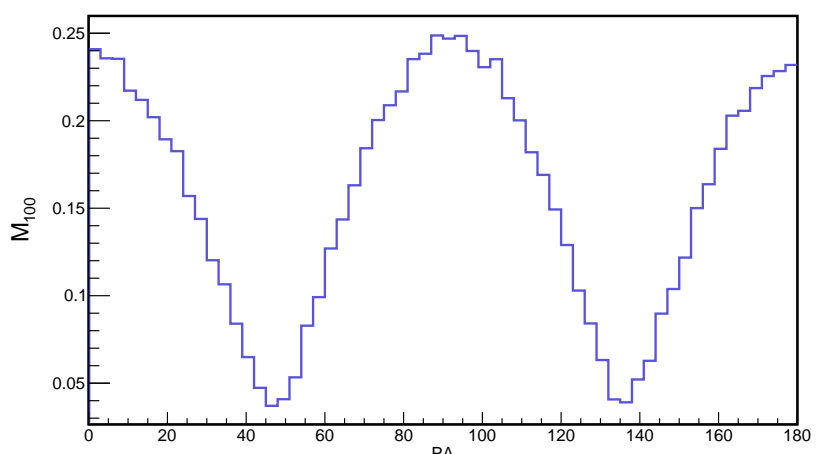

Fig. 8. Simulated $M_{100}$ value (as defined in the main text of this section) for a GRB entering POLAR with an off-axis angle of $85^{\circ}$ as a function of the PA (measured in the POLAR coordinate system). It can be seen that the sensitivity for polarization measurements is almost zero for PAs of $45^{\circ}$ and $135^{\circ}$, which is a result of the large off-axis angle of such a GRB.
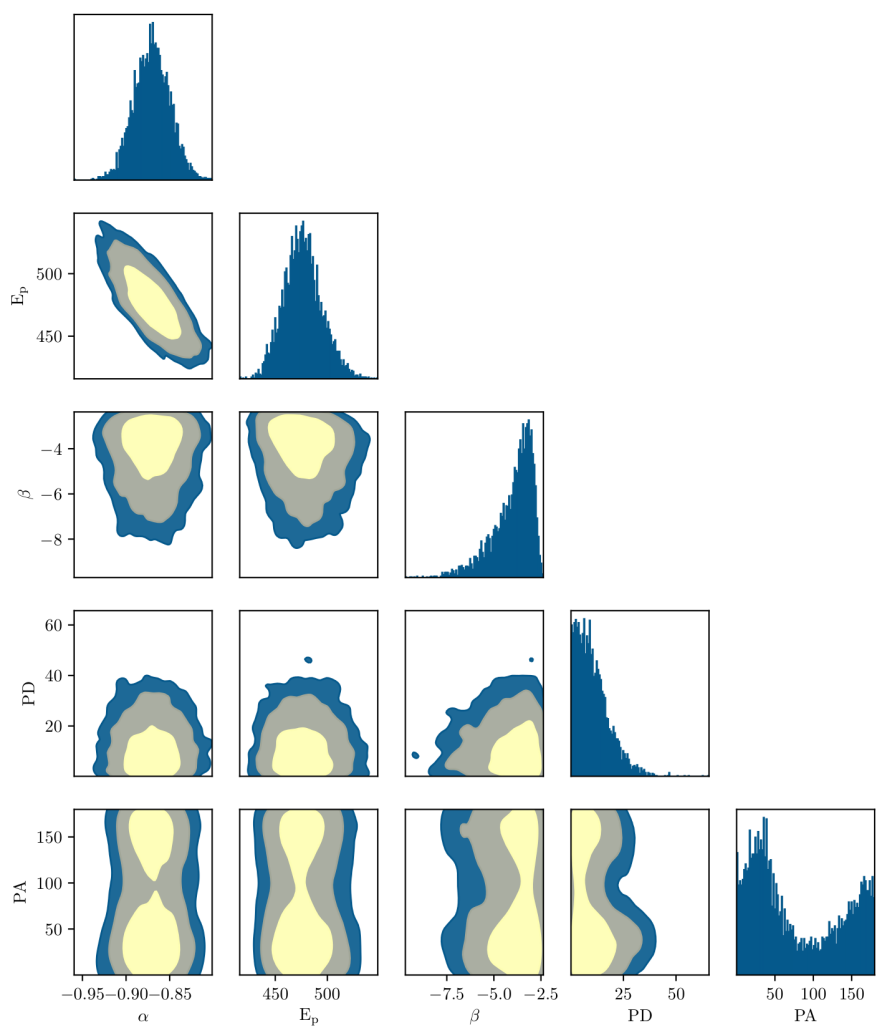

Fig. 9. Spectral and polarization posterior distributions for GRB 170207A. The $1 \sigma$ and $2 \sigma$ credibility intervals as well as that corresponding to $99 \%$ are indicated. The polarization angle shown here is in the POLAR coordinate system; a rotation in the positive direction of 176 degrees transforms this to the coordinate system as defined by the IAU.

\section{Discussion}

The time-integrated results presented here indicate that the PD of the GRB prompt emission in the energy range of approximately 30 to $750 \mathrm{keV}$ is unpolarized or mostly unpolarized. This result is found consistently for short and long GRBs, and multipeak and single-peak GRBs. The results are therefore in contradiction to the results reported by the AstroSat CZT-Imager collaboration presented by Chattopadhyay et al. (2019) where polarization levels of typically around 50\% are found and an
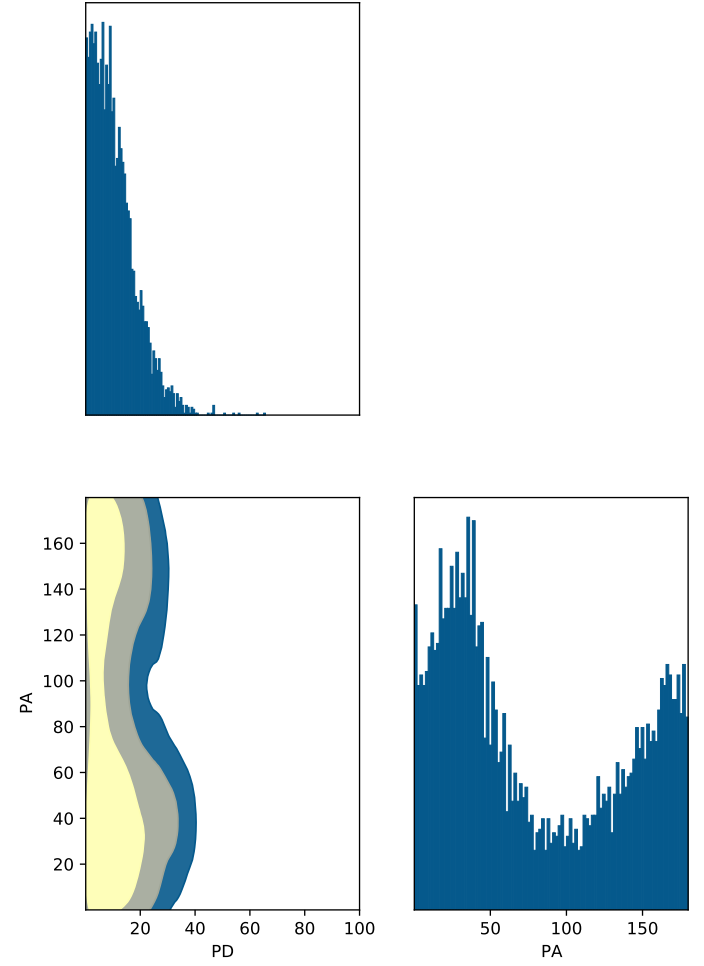

(a)

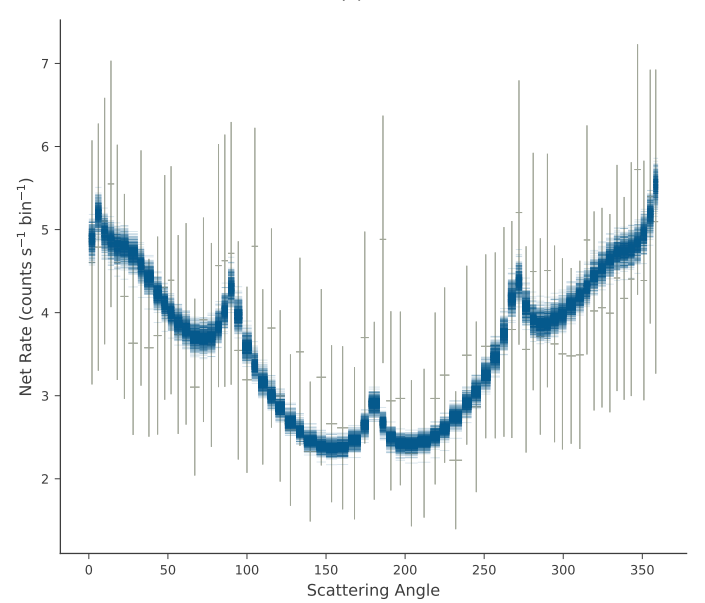

(b)

Fig. 10. Panel a: polarization posterior distributions for GRB 170207A with the 1 and $2 \sigma$ credibility intervals as well as that corresponding to 99\% credibility. The polarization angle shown here is in the POLAR coordinate system; a rotation in the positive direction of 176 degrees transforms this to the coordinate system as defined by the IAU. Panel $b$ : measured scattering angle distribution (gray data points with a 5 degree bin size) with the posterior model predictions superimposed in red. The errors on the data points are the Poisson errors corrected for the background.

unpolarized flux is excluded with $3 \sigma$ certainty for several GRBs. Future joint polarization analyses for GRBs observed by both instruments, which are possible using the 3ML framework, are vital to deciphering the origin of these incompatible results. As the AstroSat mission has reported an observation for GRB 161218B (Kumar et al. 2017) for which we here exclude a high level of polarization for the majority of polarization angles, this GRB forms a prime target for such joint analyses in the future. 

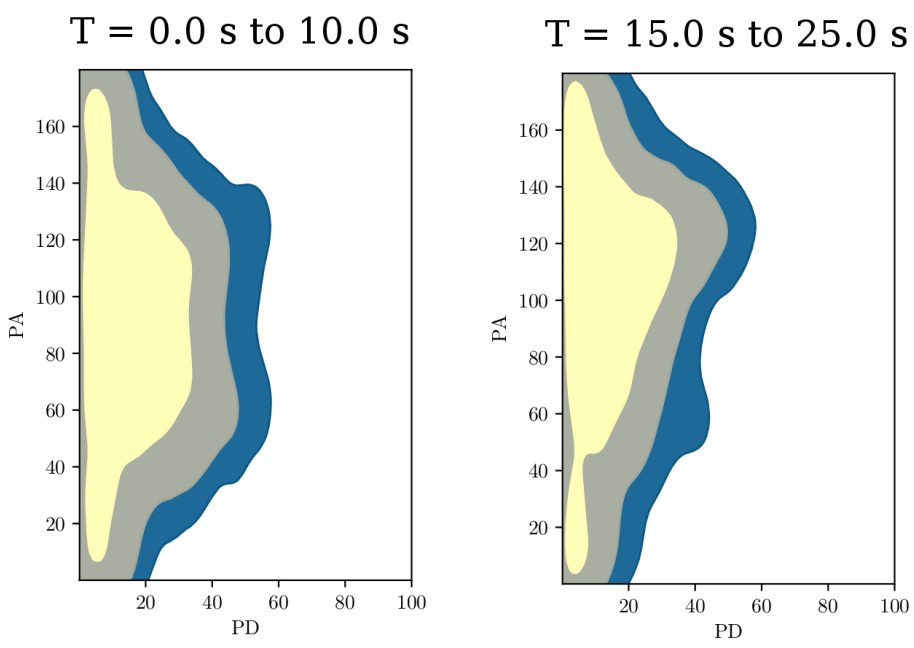

Fig. 11. Polarization posterior distributions for the two time intervals studied for GRB 170207A are shown with the $1 \sigma$ and $2 \sigma$ credibility intervals as well as that corresponding to $99 \%$ credibility. On the left, the posteriors are for the first selected time interval ranging from $T=$ $0.0 \mathrm{~s}$ to $T=10.0 \mathrm{~s}$ (corresponding to the first emission period), while on the right the posteriors are for the second time bin ranging from $T=15.0 \mathrm{~s}$ to $T=25.0 \mathrm{~s}$ (corresponding to the second emission period). The polarization angle shown here is in the POLAR coordinate system; a rotation in the positive direction of 176 degrees transforms this to the coordinate system as defined by the IAU.

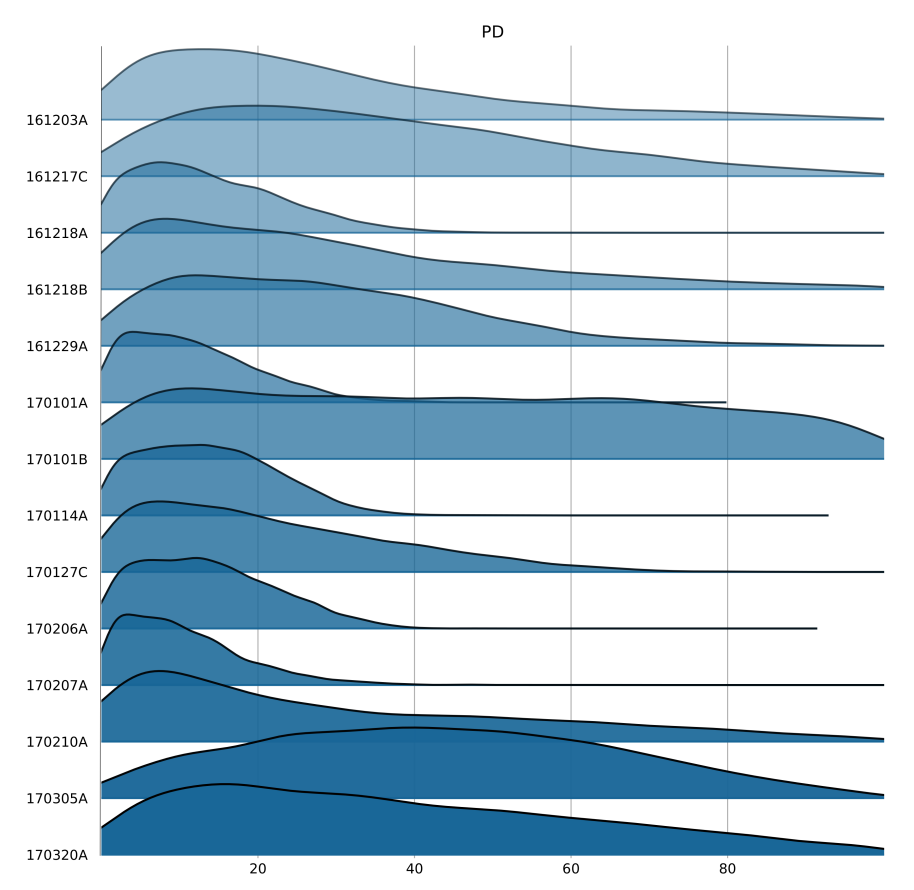

Fig. 12. Posterior distributions of the polarization degree of the 14 GRBs studied in this work.

These POLAR results appear to disfavor synchrotron models with globally ordered toroidal magnetic fields (Gill et al. 2020). However, the time-integrated results are consistent with synchrotron emission with a radial or normal to the radial magnetic field as well as Compton drag models. Although high values for PD can be produced in special cases in such models, polarization measurements for a sample of GRBs would result in a distribution peaking at $0 \%$ (Gill et al. 2020). Additionally, the results are compatible with photospheric emission which predicts

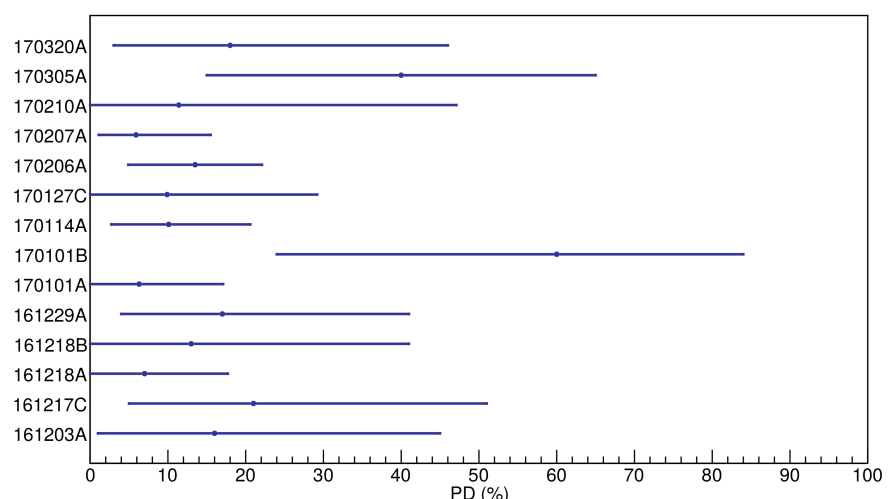

Fig. 13. Overview of the polarization degrees found for the 14 GRBs analyzed here. The errors shown are those corresponding to a $68 \%$ credibility.

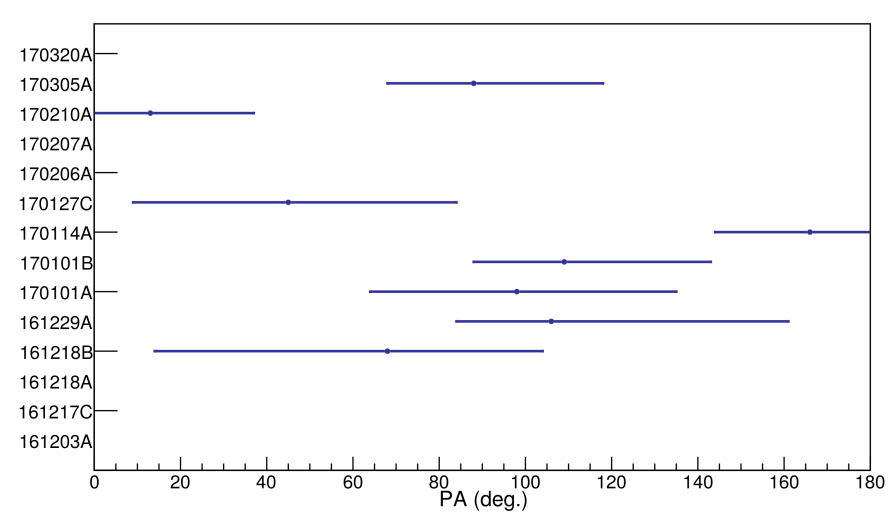

Fig. 14. Overview of the polarization angles, with an angle definition following the IAU convention, for the 14 GRBs analyzed here. The errors shown are those corresponding to a $68 \%$ credibility. No value is provided for GRBs for which no constraining PA was found (meaning that the total $1 \sigma$ error exceeds $180^{\circ}$ ).

PD distributions peaking at $0 \%$ and not exceeding $40 \%$ (Lundman et al. 2014). The time-integrated results presented here therefore only exclude synchrotron emission with globally ordered toroidal magnetic fields.

The results additionally agree with the predictions of the majority of models regarding the evolution of the PD and PA over multiple pulses (Gill et al. 2020), as no significant PD is found during time-resolved analyses where pulses are separated. However, the results presented here do support claims of intrapulse evolution found for GRB 170114A from previous studies and also reveal an additional though weaker signature of this behavior for GRB 170101A. For all GRBs for which intra-pulse time-resolved studies were possible, PD values of around $30 \%$ are found with an evolving PA. We are not aware of any theoretical work performed on the evolution of the PA within singlepulse GRBs and therefore encourage the community to provide predictions for this.

It should finally be noted that, although the work presented here contains the vast majority of all the information which can be extracted from the POLAR mission regarding GRBs, energy-resolved studies have not yet been performed. Such studies have the possibility to for example test predictions such as those made in Lundman et al. (2016) for photospheric emission. In such models, the PD for the low-energy emission (tens of $\mathrm{keV}$ ) can be relatively high, while at higher 
Table 2. Length, fluence, location, and polarization properties of the 14 GRBs resulting from the analysis presented in this work.

\begin{tabular}{lccccccc}
\hline \hline GRB & T90 $(\mathrm{s})$ & Fluence $^{(*)}$ & PD $(\%)$ & PA (deg) & $\theta$ & RA (deg) & Dec (deg) \\
\hline $161203 \mathrm{~A}$ & $4.1 \pm+0.1$ & $(7.84 \pm 1.05) \times 10^{-6}$ & $16_{-15}^{+29}$ & $\mathrm{x}$ & $85^{\circ}$ & $13.5^{\circ}$ & $-146^{\circ}$ \\
$161217 \mathrm{C}$ & $6.3 \pm+0.3$ & $(4.37 \pm 1.05) \times 10^{-6}$ & $21_{-16}^{+30}$ & $\mathrm{x}$ & $35^{\circ}$ & $34.6^{\circ}$ & $-4.0^{\circ}$ \\
$161218 \mathrm{~A}$ & $11.5 \pm+0.1$ & $(8.72 \pm 1.44) \times 10^{-6}$ & $7.0_{-7.0}^{+10.7}$ & $\mathrm{x}$ & $24.3^{\circ}$ & $245.3^{\circ}$ & $-4.1^{\circ}$ \\
$161218 \mathrm{~B}$ & $25.1 \pm+0.2$ & $(8.55 \pm 0.30) \times 10^{-5}$ & $13_{-13}^{+28}$ & $68_{-54}^{+36}$ & $80.5^{\circ}$ & $0.9^{\circ}$ & $-14.7^{\circ}$ \\
$161229 \mathrm{~A}$ & $31.3 \pm+0.4$ & $(4.21 \pm 0.22) \times 10^{-5}$ & $17_{-13}^{+24}$ & $106_{-22}^{+52}$ & $87.6^{\circ}$ & $78.9^{\circ}$ & $45.7^{\circ}$ \\
$170101 \mathrm{~A}$ & $2.0 \pm+0.1$ & $(6.8 \pm 1.4) \times 10^{-6}$ & $6.3_{-6.3}^{+10.8}$ & $98_{-34}^{+37}$ & $6.0^{\circ}$ & $276.1^{\circ}$ & $11.7^{\circ}$ \\
$170101 \mathrm{~B}$ & $11.1 \pm+0.4$ & $(1.2 \pm 0.12) \times 10^{-5}$ & $60_{-36}^{+24}$ & $109_{-21}^{+34}$ & $75.0^{\circ}$ & $69.6^{\circ}$ & $-1.0^{\circ}$ \\
$170114 \mathrm{~A}$ & $10.4 \pm+0.2$ & $(1.84 \pm 0.30) \times 10^{-5}$ & $10.1_{-7.4}^{+10.5}$ & $166_{-22}^{+25}$ & $26.4^{\circ}$ & $13.1^{\circ}$ & $-13.0^{\circ}$ \\
$170127 \mathrm{C}$ & $0.14 \pm+0.01$ & $(5.4 \pm 2.4) \times 10^{-6}$ & $9.9_{-8.3}^{+19.3}$ & $45_{-36}^{+39}$ & $41.8^{\circ}$ & $339.3^{\circ}$ & $-63.9^{\circ}$ \\
$170206 \mathrm{~A}$ & $1.26 \pm+0.01$ & $(1.04 \pm 0.06) \times 10^{-5}$ & $13.5_{-8.6}^{+7.4}$ & $\mathrm{x}$ & $19.5^{\circ}$ & $212.8^{\circ}$ & $14.5^{\circ}$ \\
$170207 \mathrm{~A}$ & $38.8 \pm+0.3$ & $(6.80 \pm 0.47) \times 10^{-5}$ & $5.9_{-5.9}^{+9.6}$ & $\mathrm{x}$ & $67.2^{\circ}$ & $316.9^{\circ}$ & $59.1^{\circ}$ \\
$170210 \mathrm{~A}$ & $47.6 \pm+2.5$ & $(7.21 \pm 0.28) \times 10^{-5}$ & $11.4_{-9.7}^{+3.7}$ & $13_{-21}^{+24}$ & $80.6^{\circ}$ & $226.1^{\circ}$ & $-65.1^{\circ}$ \\
$170305 \mathrm{~A}$ & $0.45 \pm+0.01$ & $(1.08 \pm 0.12) \times 10^{-6}$ & $40_{-25}^{+25}$ & $88_{-20}^{+30}$ & $31.4^{\circ}$ & $39.7^{\circ}$ & $9.9^{\circ}$ \\
$170320 \mathrm{~A}$ & $6.8 \pm+0.1$ & $(9.6 \pm 4.8) \times 10^{-6}$ & $18_{-18}^{+32}$ & $\mathrm{x}$ & $84.7^{\circ}$ & $320.1^{\circ}$ & $55.1^{\circ}$ \\
\hline
\end{tabular}

Notes. The $T_{90}$ parameter as calculated using POLAR data are provided along with the off-axis incoming angle $(\theta)$ and the RA (J2000) and Dec (J2000) positions used in the analysis. For the RA (J2000) and Dec (J2000) parameters we report those used to produce the instrument response in the analysis. In cases where the PA, which is given according to the IAU convention, is not constrained (meaning that the total $1 \sigma$ error exceeds $\left.180^{\circ}\right)$ an $\mathrm{x}$ is added in this table. ${ }^{(*)}$ in units of $\mathrm{erg} \mathrm{cm}^{-2}$ in $10-1000 \mathrm{keV}$.

energies the PD is lost due to comptonization of this emission component. Whilst this analysis appears straightforward, the energy dispersion of gamma-ray detectors requires the development of dedicated analysis techniques. Such studies are therefore expected to be performed with existing POLAR data in the future.

Acknowledgements. We gratefully acknowledge the financial support from the National Natural Science Foundation of China (Grant No. 11961141013, 11503028), the Joint Research Fund in Astronomy under the cooperative agreement between the National Natural Science Foundation of China and the Chinese Academy of Sciences (Grant No. U1631242), the Xie Jialin Foundation of the Institute of High Energy Phsyics, Chinese Academy of Sciences (Grant No. 2019IHEPZZBS111), the National Basic Research Program (973 Program) of China (Grant No. 2014CB845800), the Strategic Priority Research Program of the Chinese Academy of Sciences (Grant No. XDB23040400), the Swiss Space Office of the State Secretariat for Education, Research and Innovation (ESA PRODEX Programme), the National Science Center of Poland (Grant No. 2015/17/N/ST9/03556), and the Youth Innovation Promotion Association of Chinese Academy of Sciences (Grant No. 2014009). J.M. Burgess acknowledges support from the Alexander von Humboldt Foundation. The authors are grateful to the Fermi-GBM team and HEASARC for public access to Fermi data products and to the Swift-BAT team for public access to their data products. Specifically, we would like to thank Dr. Amy Lien for assistance with the analysis of the Swift-BAT data. Finally, we are extremely thankful for the anonymous referee who went through the paper in extreme detail and who, through many suggestions, helped to significantly improve this work.

\section{References}

\section{Abbott, B. P. 2019, ApJ, 848, L13}

Ackermann, M., Ajello, M., Asano, K., et al. 2013, ApJS, 209, 11

di Serego Alighieri, S. 2017, Exp. Astron., 43, 19

Band, D., Matteson, J., Ford, L., et al. 1993, ApJ, 413, 2

Barthelmy, S. D., Cummings, J. R., Gehrels, N., et al. 2017, GCN Circular 20353, https://gcn.gsfc.nasa.gov/other/170101A.gcn3

Berlato, F., Greiner, J., \& Burgess, J. M. 2019, ApJ, 873, 60

Bissaldi, E., Mailyan, B., Meegan, C., et al. 2017, GCN Circular 20543, https : //gcn.gsfc.nasa.gov/other/170127C.gcn3

Buchner, J., Georgakakis, A., Nandra, K., et al. 2014, A\&A, 564, A125

Burgess, J. M. 2019, A\&A, 629, A69

Burgess, J. M., Ryde, F., Yu, H.-F., et al. 2015, MNRAS, 451, 1511

Burgess, J. M., Kole, M., Berlato, F., et al. 2019, A\&A, 627, A105

Chauvin, M., Florén, H. G., Friis, M., et al. 2017, Scientific Reports, 7, 7816
Chattopadhyay, T., Vadawale, S. V., Aarthy, E., et al. 2019, ApJ, 884, A123

Covino, S., \& Götz, D. 2016, Astron. Astrophys. Trans., 29, 205

Fana Dirirsa, F., Tak, D., Vianello, G., et al. 2017, GCN Circular 20617, https: //gcn.gsfc.nasa.gov/other/170206A.gcn3

Fermi Collaboration 2016, Fermi Notice for Trigger 504738232, https : //gcn. gsfc.nasa.gov/other/504738232. fermi

Feroz, F., Hobson, M. P., \& Bridges, M. 2009, MNRAS, 398, 1601

Frederiks, D., Golenetskii, S., Aptekar, R., et al. 2017, GCN Circular 20567, https://gcn.gsfc.nasa.gov/other/170127C.gcn3

Gehrels, N., \& Razzaque, S. 2013, Front. Phys., 8, 661

Gill, R., Granot, J., Kumar, P., et al. 2020, MNRAS, 491, 3343

Hamburg, R., Meegan, C., Yu, H., et al. 2016, GCN Circular 20286, https: //gcn.gsfc.nasa.gov/other/161218B.gcn3

Hurley, K., et al. (on behalf of the IPN team) 2017a, GCN Circular 20661, https://gcn.gsfc.nasa.gov/other/170210A.gcn3

Hurley, K., et al. (on behalf of the IPN team) 2017b, GCN Circular 20824, https://gcn.gsfc.nasa.gov/other/170305A.gcn3

Hurley, K., et al. (on behalf of the IPN team) 2017c, GCN Circular 20927. https://gcn.gsfc.nasa.gov/other/170320A.gcn3

Kole, M., Produit, N., Bernasconi, T., et al. 2016, GCN Circular 20243, https: //gcn.gsfc.nasa.gov/other/161203A.gcn3

Kole, M., Li, Z. H., Produit, N., et al. 2017, Nucl. Instrum. Meth. Phys. Res. A, 872,28

Kole, M. 2019, POLAR-2: The First Large Scale Gamma-ray Polarimeter (ICRC 2019), 572

Konus 2016a, Konus-Wind Report of 161203A, http: //www . ioffe.ru/LEA/ kw/triggers/2016/kw20161203_67266.html

Konus 2016b, Konus-Wind Report of 161203A, http: //www . ioffe.ru/LEA/ $\mathrm{kw} /$ triggers/2016/kw20161217_13995.html

Kumar, V., Bhattacharya, D., Bhalerao, V., et al. 2017, GCN Circular 20324, https://gcn.gsfc.nasa.gov/other/161218B.gcn3

LEAP Collaboration (McConnell, M. L., et al.) 2016, LEAP - A LargE Area Burst Polarimeter for the ISS, 4051

Lien, A., Sakamoto, T., Barthelmy, S. D., et al. 2016, ApJ, 829, A7

Li, H. C., Ge, M., Wu, B., et al. 2017, POLAR Measurements of the Crab Pulsar PoS (ICRC 2017)

Li, Z. H., Kole, M., Sun, J., et al. 2018, Nucl. Inst. Meth. Phys. Res. A, 900C, 8

Li, H. C., Gauvin, N., Ge, M.-Y., et al. 2019, J. High Energy Astrophys., 24, 15

Lundman, C., Peér, A., \& Ryde, F. 2014, MNRAS, 440, 3292

Lundman, C., Vurm, I., \& Beloborodov, A. M. 2016, ArXiv e-prints [arXiv:1611.01451]

Luytikov, M., Pariev, V. I., Blandford, R. D., et al. 2003, ApJ, 597, 998

Markwardt, C. B., Barthelmy, S. D., Cummings, J. R., et al. 2016, GCN Circular 20293, https://gcn.gsfc.nasa.gov/other/161218A.gcn3

Mazets, E. P., Golenetskii, S. V., Il'Inskii, V. N., et al. 1981, Astrophys. Space Sci., 80,3 
McConnell, M. L. 2017, New Astron. Rev., 76, 1

Meegan, A. C. 1997, The Fourth BATSE Gamma-ray Burst Catalog GRBs: The Fourth Huntsville Symposium

Moretti, E., Vianello, G., Bissaldi, E., et al. 2017, GCN Circular 20544, https : //gcn.gsfc.nasa.gov/other/170127C.gcn3

Produit, N., Baod, T. W., Batsch, T., et al. 2018, Nucl. Instrum. Meth. Phys. Res A, 877,259

RHESSI Collaboration 2020, RHESSI Solar Flare Catalog, https: //hesperia.gsfc.nasa.gov/hessidata/dbase/hessi_flare_list. txt

Roberts, O. J., \& Mailyan, B. 2017, GCN Circular 20660, https: //gcn.gsfc nasa.gov/other/170210A.gcn3

Roberts, O. J., \& Mailyan, B. (on behalf of the GBM team) 2017, GCN Circular 20630, https://gcn.gsfc.nasa.gov/other/170207A.gcn3

Sharma, V., Iyyani, S., Bhattacharya, D., et al. 2019, ApJ, 882, L10

Sharma, V., Iyyani, S., Bhattacharya, D., et al. 2020, MNRAS, 493, 518

Spiegelhalter, D. J. 2002, J. R. Stat. Soc. B, 64, 4

Stanbro, M., von Kienlin, A., Meegan, C., et al. 2017, GCN Circular 20818, https://gcn.gsfc.nasa.gov/other/170305A.gcn3

Suarez Garcia, E. 2010, PhD Thesis, University of Geneva

Svinkin, D., Golenetskii, S., Aptekar, R., et al. 2016, GCN Circular 20303 https://gcn.gsfc.nasa.gov/other/161218B.gcn3

Toma, K., Sakamoto, T., Zhang, B., et al. 2009, ApJ, 698, 1042

Tsvetkova, A., Golenetskii, S., Aptekar, R., et al. 2017a, ApJ, 850, 161

Tsvetkova, A., Golenetskii, S., Aptekar, R., et al. 2017b, GCN Circular 20935, https://gcn.gsfc.nasa.gov/other/170320A.gcn3
Veres, P., \& Meegan, C. (on behalf of the GBM team) 2017a, GCN Circular 20350, https://gcn.gsfc.nasa.gov/other/1701010B.gcn3

Veres, P. (on behalf of the GBM team) 2017b, GCN Circular 20461, https: //gcn.gsfc.nasa.gov/other/170114A.gcn3

von Kienlin, A., \& Roberts, O. J., et al. ( on behalf of the GBM team) 2017, GCN Circular 20616, https://gcn.gsfc .nasa.gov/other/170206A.gcn3 von Kienlin, A., Meegan, C. A., Paciesas, W. S., et al. 2020, ApJ, 893, 46

Vianello, G., Lauer, R. J., Younk, P., et al. 2015, ArXiv e-prints [arXiv:1507.08343]

Wang, Y. H. \& Xiong, S. L. (on behalf of the POLAR team) 2016, GCN Circular 20311, https://gcn.gsfc.nasa.gov/other/161217C.gcn3

Wang, Y. H., Sun, J. C., Kole, M., et al. 2020, Nucl. Instrum. Meth. Phys. Res A, submitted

Woosley, S. E. 1993, ApJ, 405, 273

Xiao, H. L., Hajdasa, W., Wu, B., et al. 2018, Astropart. Phys., 103, 74

Xiong, S. L., Wangab, Y. H., Li, Z. H., et al. 2017, Overview of the GRB observation by POLAR

Yonetoku, D. 2013, https://ttt.astro.su.se/groups/head/cost14/ talks/Yonetoku.pdf

Yassine, M., Piron, F., Daigne, F., et al. 2020, A\&A, 640, A91

Yonetoku, D., Murakami, T., Gunji, S., et al. 2011, ApJ, 743, L30

Zhao, Y., Wang, Y. H., Li, Z. H. (on behalf of the POLAR team) 2017, GCN Circular 20934, https://gcn. gsfc.nasa.gov/other/170320A.gcn3

Zhang, B., \& Yan, H. 2011, ApJ, 726, 90

Zhang, S. N., Kole, M., Bao, T.-W., et al. 2019a, Nat. Astron., 3

Zhang, B. B., Lucas Uhm, Z., Connaughton, V., et al. 2019b, ApJ, 816 


\section{Appendix A: 161203A}

The GRB 161203A was detected by POLAR on December 3, 2016 at 18:41:07.75 (UT) (Kole et al. 2016), which is taken to be $T 0$ for the analysis presented here. A $T_{90}$ of $(4.1 \pm 0.1) \mathrm{s}$ was measured using POLAR data. The light curve, including the signal region (blue) and part of the background region (yellow) can be seen in Fig. A.1. It was not detected by Fermi-GBM or SwiftBAT. The GRB was detected by Konus-Wind (Konus 2016a), but no spectral parameters were reported and therefore no priors could be used. Furthermore, no location was reported for this GRB by any instrument. For this purpose, the POLAR localization method reported in Wang et al. (2020) was used to calculate a relative position for POLAR corresponding to $\theta=85^{\circ}$ and $\phi=170^{\circ}$ where $\theta$ is the angle with respect to the zenith of POLAR and $\phi$ is the angle with respect to the $x$-axis of POLAR (which corresponds to a scattering angle of $0^{\circ}$ ). These coordinates correspond to RA $(\mathrm{J} 2000)=194^{\circ}$ and $\operatorname{Dec}(\mathrm{J} 2000)=-34^{\circ}$ with an uncertainty of $7^{\circ}$. The spectral results from the joint fit can be seen in Fig. A.1.

The joint spectral and polarization fit for GRB 161203A was performed using POLAR data only. This makes GRB 161203A a GRB that makes use of POLAR data for the location, spectrum, and polarization. As a result the systematic errors, which are all included in the final posterior distribution, are relatively large. The posterior distributions of the spectral and polarization parameters are shown in Fig. A.2. Finally the posterior distribution of the polarization parameters is shown together with the measured scattering angle distribution with the posterior model predictions superimposed in blue in Fig. A.3. The scattering angle distributions clearly indicate the large off-axis angle resulting in the $360^{\circ}$ modulation in the distribution. Additionally, the amplitude and phase of the $360^{\circ}$ modulation, which depend on the incoming direction of the GRB, can be seen to match between the model and the data, indicating that the localization method is working properly. A PD of $16_{-15}^{+29} \%$ is found. A $99 \%$ credibility upper limit for PD of $98 \%$ is found. Although these results exclude very high polarization, no properly constraining measurement was possible, which is partly due the lack of measurements by other instruments and partly due to the lack of sensitivity for a range of polarization angles because of the large incoming off-axis angle of the GRB. It should however be noted that while the polarization is found to be consistent with a PD of $0 \%$, a high PD can be excluded with the exception of polarization angles for which POLAR was not sensitive because of the incoming angle. The signal-to-background-count ratio in

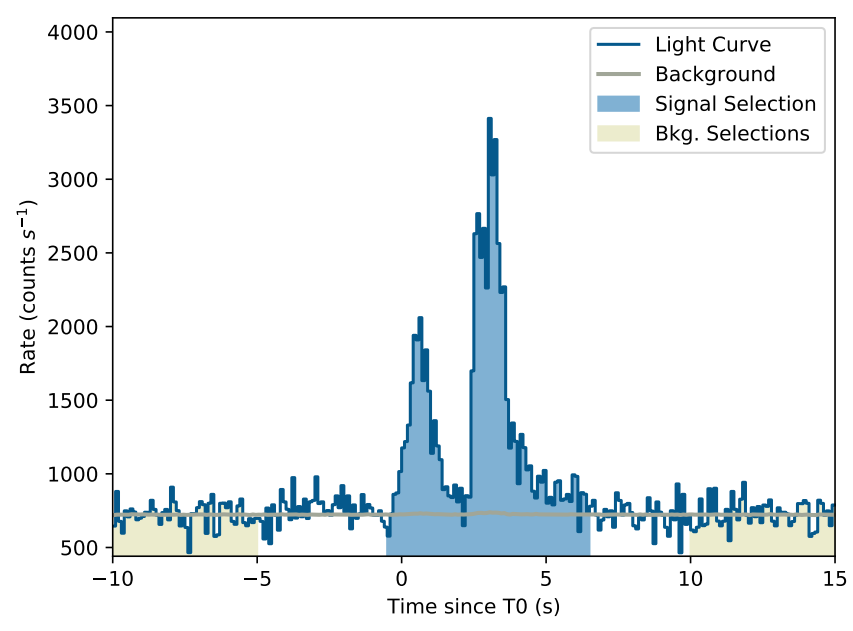

(a)

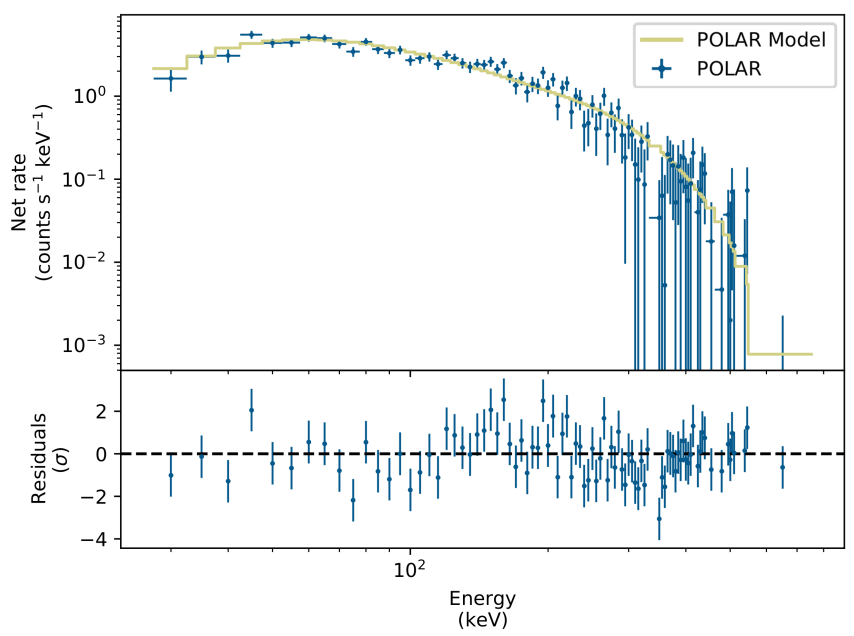

(b)

Fig. A.1. Panel a: light curve of GRB 161203A as measured by POLAR, where $T=0 \mathrm{~s}$ is defined as the detection time measured by POLAR (Kole et al. 2016). Panel b: joint spectral fit result for 161203A. The number of counts as detected by POLAR (blue) is shown along with the best-fitting spectrum folded through the instrument response in yellow. The residuals are shown at the bottom of the figure.

the POLAR data from this GRB does not allow for any timeresolved analysis. 

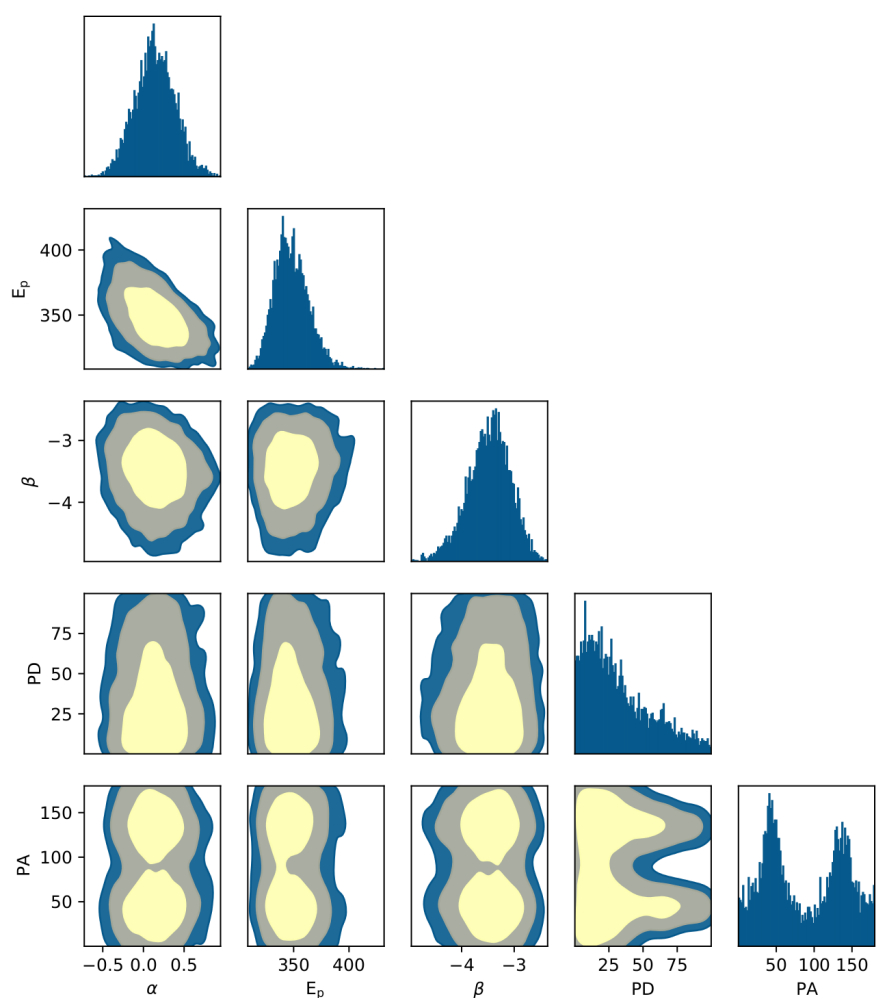

Fig. A.2. Spectral and polarization posterior distributions for GRB 161203A. The $1 \sigma$ and $2 \sigma$ credibility intervals as well as that corresponding to $99 \%$ are indicated. The PA shown here is in the POLAR coordinate system; a rotation in the positive direction of 98 degrees transforms this to the coordinate system as defined by the IAU.
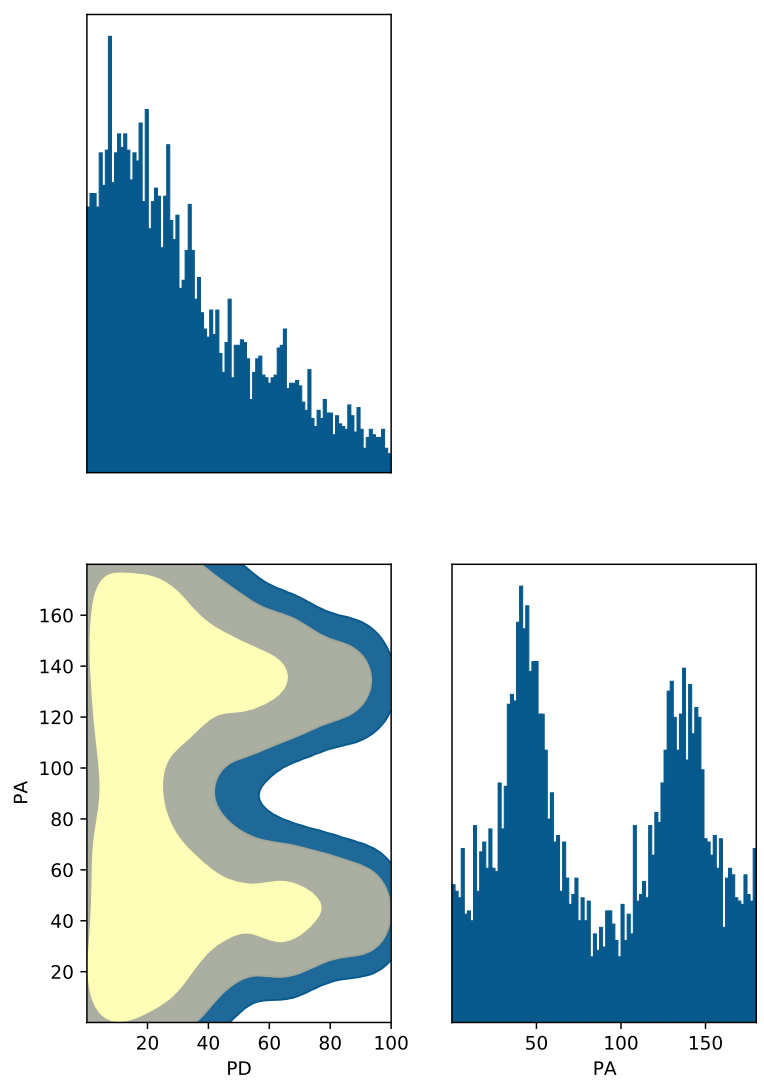

(a)

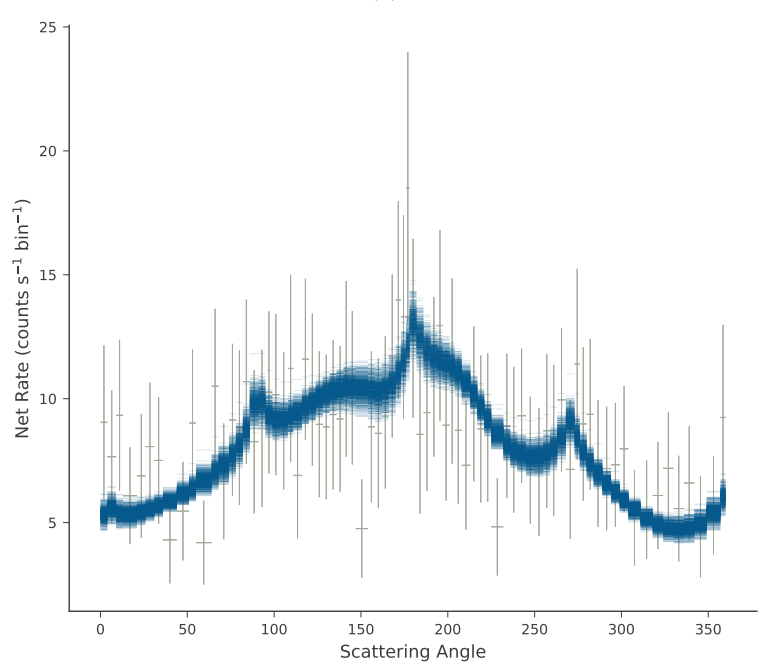

(b)

Fig. A.3. Panel a: polarization posterior distributions for GRB 161203A with the $1 \sigma$ and $2 \sigma$ credibility intervals as well as that corresponding to $99 \%$ credibility. The posterior distribution clearly reflects the lack of sensitivity for PAs of $45^{\circ}$ and $135^{\circ}$ in this measurement. The polarization angle shown here is in the POLAR coordinate system; a rotation in the positive direction of 98 degrees transforms this to the coordinate system as defined by the IAU. Panel $b$ : measured scattering angle distribution (gray data points with a 5 degree bin size) with the posterior model predictions superimposed in blue. The errors on the data points are the Poisson errors corrected for the background. 


\section{Appendix B: 161217C}

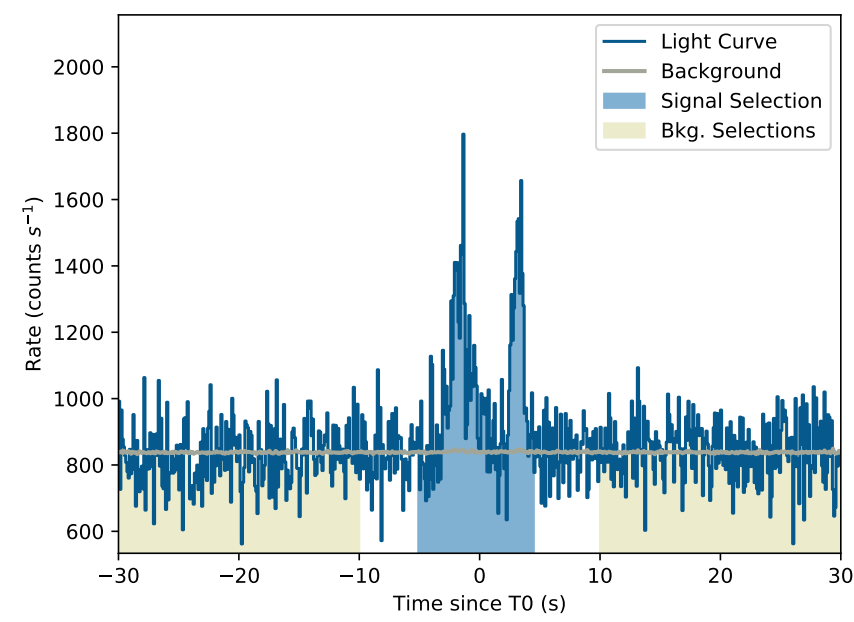

(a)

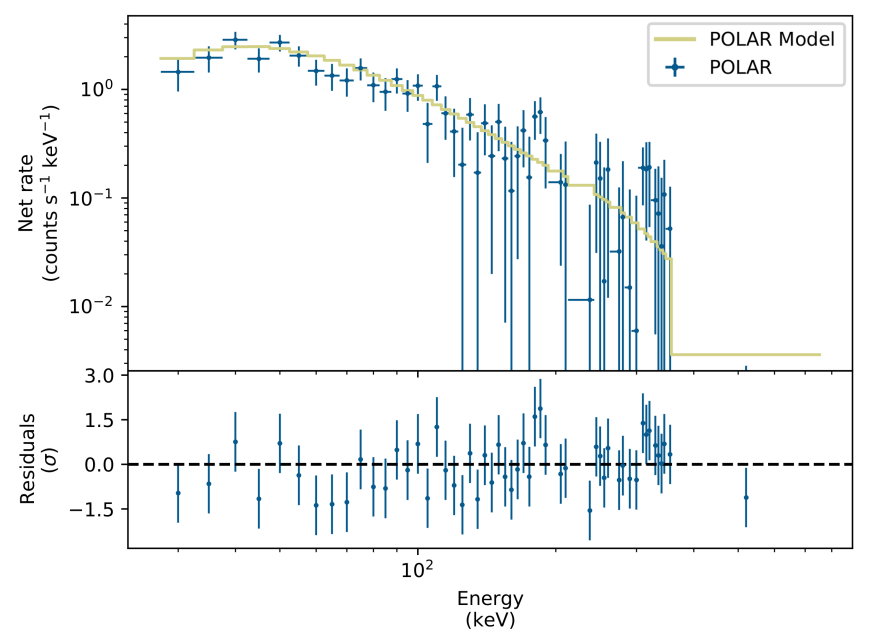

(b)

Fig. B.1. Panel a: light curve of GRB $161217 \mathrm{C}$ as measured by POLAR, where $T=0 \mathrm{~s}$ is defined as the detection time by POLAR (Wang et al. 2016). Panel $b$ : joint spectral fit result for $161217 C$. The number of counts as detected by POLAR (blue) is shown along with the best-fitting spectrum folded through the instrument response in yellow. The residuals are shown at the bottom of the figure.

The GRB 161217C was detected by POLAR on December 17, 2016 at 03:53:15 (UT) Wang et al. (2016) which is used as T0 in the analysis presented here. A $T_{90}$ of $(6.3 \pm 0.25) \mathrm{s}$ was measured using POLAR data. The light curve, including the signal region (blue) and part of the background region (yellow) can be seen in Fig. B.1. As it was not detected by Fermi-GBM or Swift-BAT, no spectral data from these instruments was available. Additionally, although the GRB was detected by KonusWind (Konus 2016b), no spectral parameters were reported and therefore no priors could be used. Furthermore, no location was reported for this GRB by any instrument. For this purpose the POLAR localization method reported in Wang et al. (2020) was
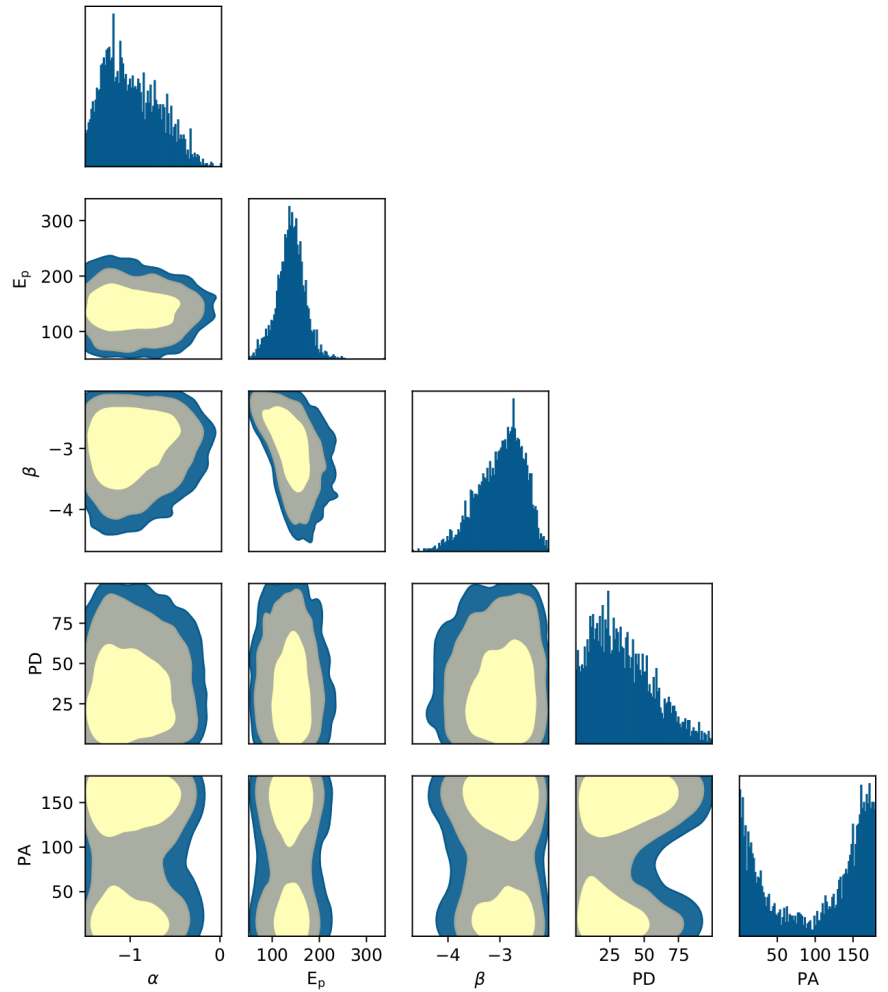

Fig. B.2. Spectral and polarization posterior distributions for GRB $161217 \mathrm{C}$. The $1 \sigma$ and $2 \sigma$ credibility intervals as well as that corresponding to $99 \%$ are indicated. The polarization angle shown here is in the POLAR coordinate system; a rotation in the positive direction of 34 degrees transforms this to the coordinate system as defined by the IAU.

used to calculate a relative position for POLAR corresponding to $\theta=35^{\circ}$ and $\phi=172^{\circ}$ where $\theta$ is the angle with respect to the zenith of POLAR and $\phi$ is the angle with respect to the $x$-axis of POLAR. These coordinates correspond to RA $(\mathrm{J} 2000)=34^{\circ}$ and $\operatorname{Dec}(\mathrm{J} 2000)=-4^{\circ}$ with an uncertainty of $7^{\circ}$. The polarization response of POLAR was produced using this location and includes the systematic error induced by the relatively large uncertainty. The joint spectral and polarization fit was performed using POLAR data only. This makes GRB 161217C, along with GRB 161203A, the only GRBs that only makes use of POLAR data for the location, spectrum, and polarization. As a result, the systematic errors, which are all included in the final posterior distribution, are relatively large. The spectral results of the joint fit can be seen in Fig. B.1. The posterior distributions of the spectral and polarization parameters are shown in Fig. B.2. Finally the posterior distribution of the polarization parameters is shown together with measured scattering angle distribution with the posterior model predictions superimposed in blue in Fig. B.3. A PD of $21_{-16}^{+30 \%}$ is found. A $99 \%$ credibility upper limit for PD of $94 \%$ is found. Although the results exclude a very high polarization, no properly constraining measurement was possible, which is partly due to the lack of measurements by other instruments. The signal-to-background-count ratio of POLAR for GRB do not allow for any time-resolved analysis. 

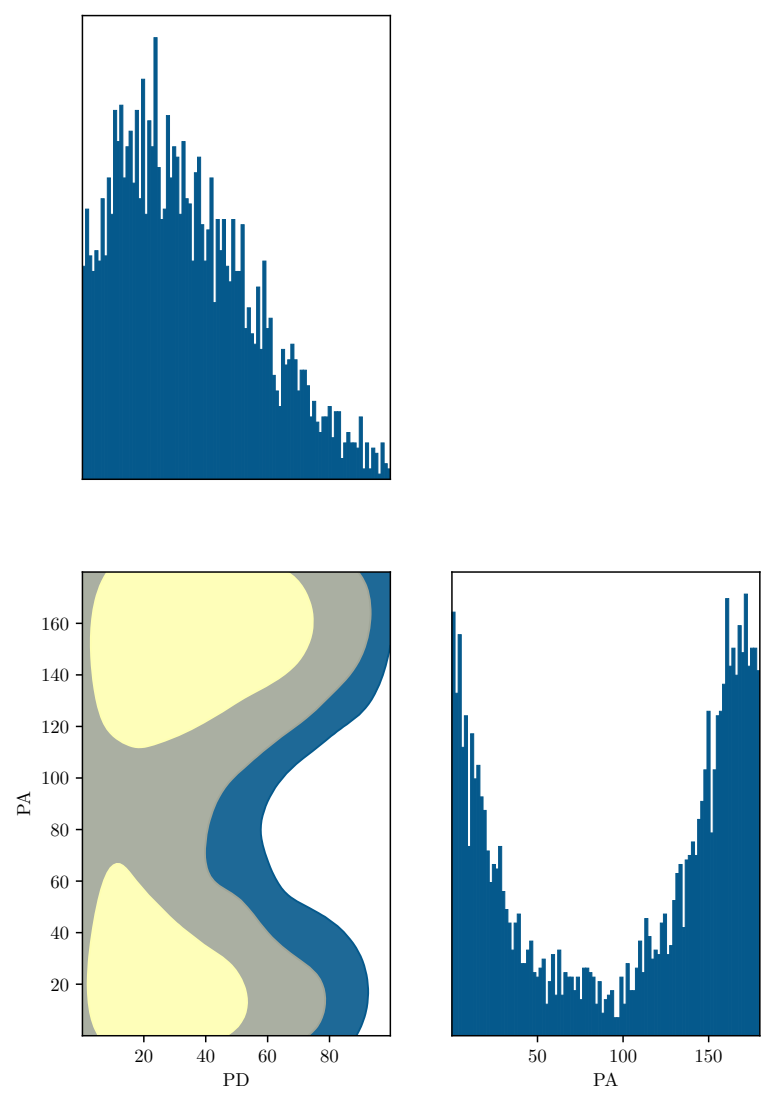

(a)

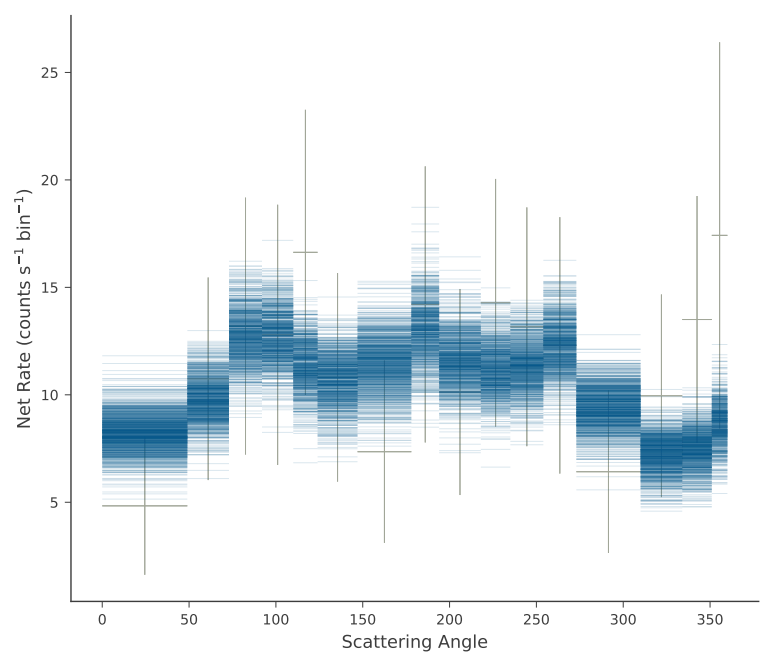

(b)

Fig. B.3. Panel a: polarization posterior distributions for GRB 161217C with the $1 \sigma$ and $2 \sigma$ credibility intervals as well as that corresponding to 99\% credibility. The PA shown here is in the POLAR coordinate system, a rotation in the positive direction of 34 degrees transforms this to the coordinate system as defined by the IAU. Panel $b$ : measured scattering angle distribution (gray data points with a 22.5 degree bin size) with the posterior model predictions superimposed in blue. The errors on the data points are the Poisson errors corrected for the background.

\section{Appendix C: 161218A}

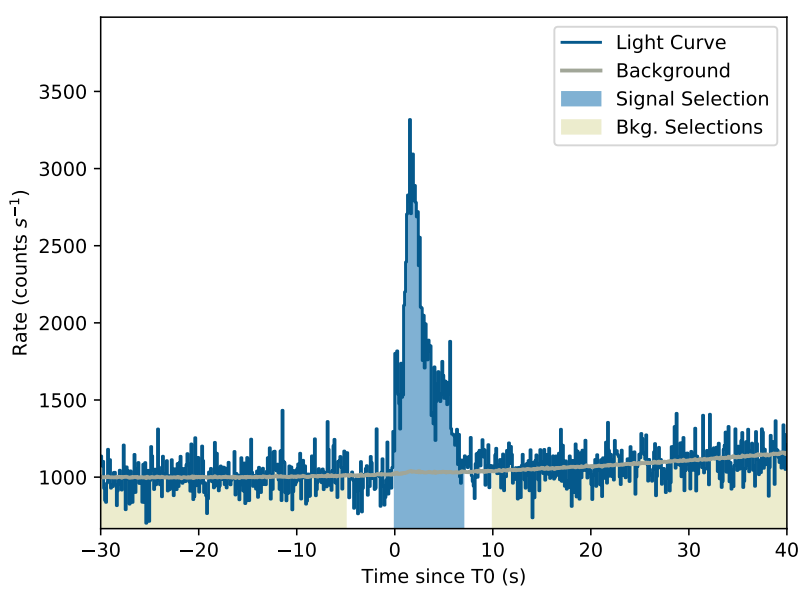

(a)

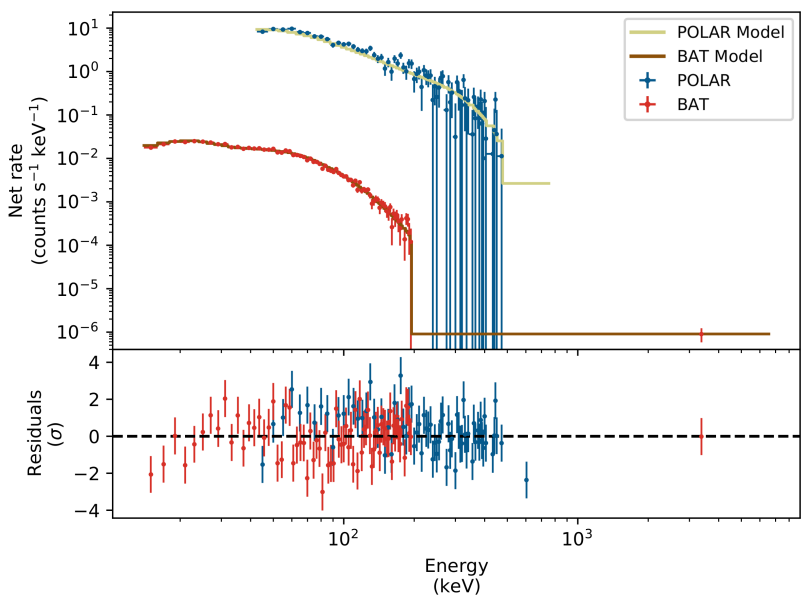

(b)

Fig. C.1. Panel $a$ : light curve of GRB 161218A as measured by POLAR, where $T=0 \mathrm{~s}$ is defined as the $T 0$ employed by Swift-BAT for this GRB in their data products. Panel $b$ : joint spectral fit result for 161218A. The number of counts as detected by both POLAR (blue) and Swift-BAT (red) are shown along with the best-fitting spectrum folded through the instrument responses in yellow for POLAR data and in brown for Swift-BAT data. The residuals for both data sets are shown at the bottom of the figure.

The GRB 161218A was detected by POLAR and by SwiftBAT (Markwardt et al. 2016) which defined a $T 0$ of December 18, 2016 at 03:47:34.75 (UT). For convenience this time is used as $T 0$ for the analysis presented here as well. A $T_{90}$ of $(11.48 \pm 0.14) \mathrm{s}$ was measured using POLAR data. The light curve from POLAR, including the signal region (blue) and part of the background region (yellow) can be seen in Fig. C.1. As it was detected by Swift-BAT, spectral data from this instrument was used to perform the joint fit. The spectral results of the joint fit for this GRB can be seen in Fig. C.1. The effective area correction (applied to the POLAR data) found in the 

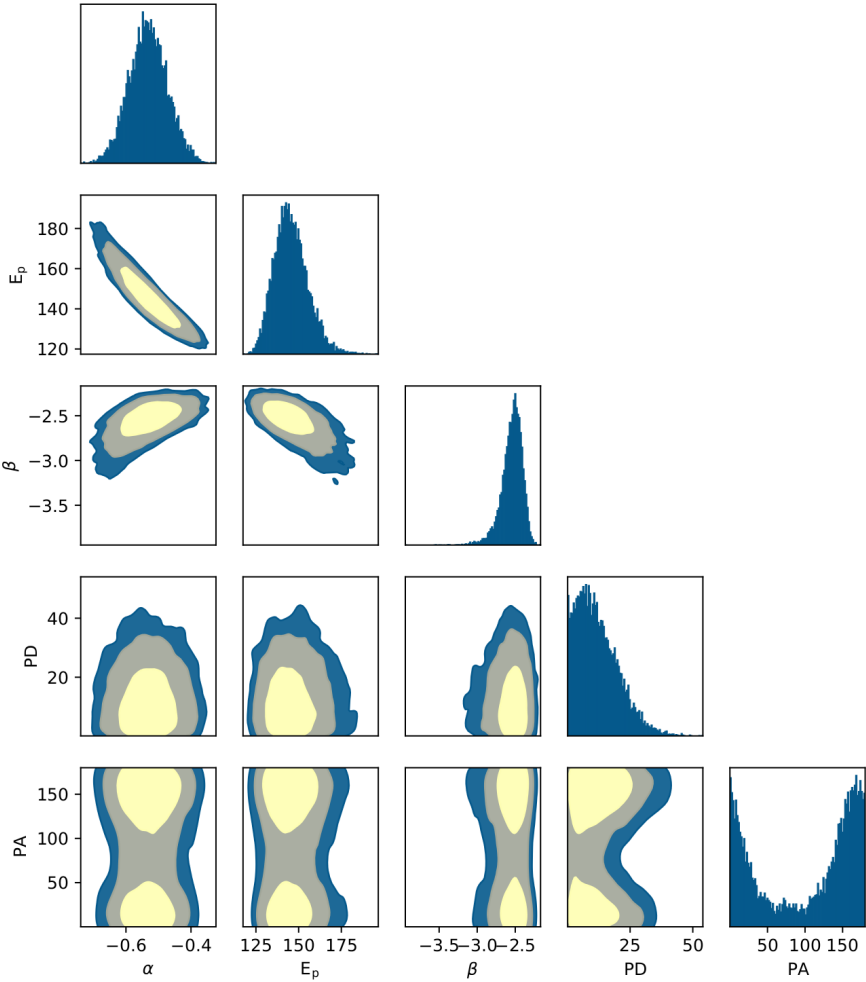

Fig. C.2. Spectral and polarization posterior distributions for GRB $161218 \mathrm{~A}$. The $1 \sigma$ and $2 \sigma$ credibility intervals as well as that corresponding to $99 \%$ are indicated. The polarization angle shown here is in the POLAR coordinate system; a rotation in the positive direction of 156 degrees transforms this to the coordinate system as defined by the IAU.

analysis is $1.08 \pm 0.05$. The spectral response of both instruments can be seen to be in good agreement. The polarization response of POLAR was produced using the refined location provided by Swift-BAT: RA $(\mathrm{J} 2000)=245.250^{\circ}$, Dec $(\mathrm{J} 2000)=-4.113^{\circ}$ (Markwardt et al. 2016). The posterior distributions of the spectral and polarization parameters are shown in Fig. C.2. Finally, the posterior distribution of the polarization parameters is shown together with the measured scattering angle distribution superimposed by the posterior model predictions (blue) in Fig. C.3. A PD of $7.0_{-7.0}^{+10.7 \%}$ is found which is compatible with that reported in Zhang et al. (2019a). A 99\% credibility upper limit for PD of $38.0 \%$ was found.
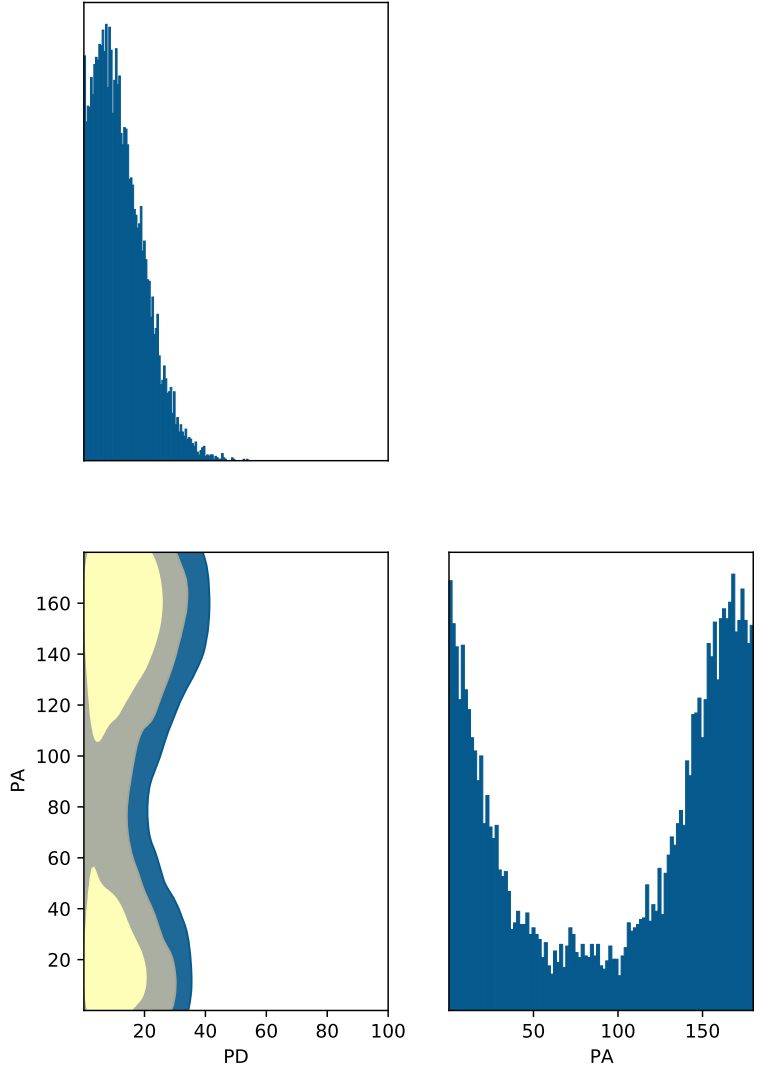

(a)

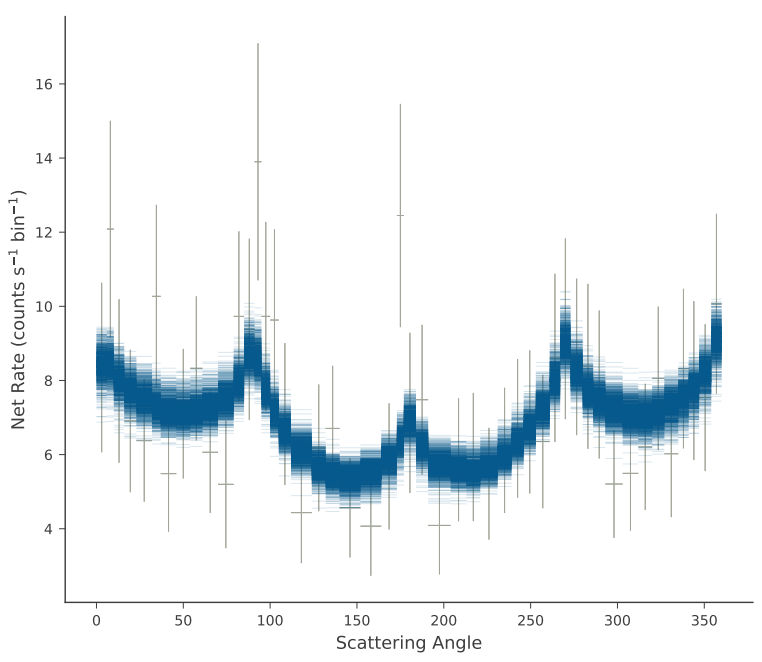

(b)

Fig. C.3. Panel a: polarization posterior distributions for GRB 161218A with the $1 \sigma$ and $2 \sigma$ credibility intervals as well as that corresponding to $99 \%$. The polarization angle shown here is in the POLAR coordinate system; a rotation in the positive direction of 156 degrees transforms this to the coordinate system as defined by the IAU. Panel $b$ : measured scattering angle distribution (gray data points with a 7.5 degree bin size) with the posterior model predictions superimposed in blue. The errors on the data points are the Poisson errors corrected for the background. 


\section{Appendix D: 161218B}

The GRB 161218B was detected by POLAR and by FermiGBM. The latter reported a T0 of December 18, 2016 at 08:32:40.65 (UT) (Hamburg et al. 2016), which is taken to be the $T 0$ for the analysis presented here for convenience. A $T_{90}$ of $(25.1 \pm 0.2) \mathrm{s}$ was measured using POLAR data. The light curve, including the signal region (blue) and part of the background region (yellow) can be seen in Fig. D.1. It should be noted here that the GRB was detected by POLAR while the instrument was at a relatively high magnetic latitude. This resulted in an aboveaverage background rate which, additionally, showed some significant variations with time as can be seen in the light curve. Details on the background conditions of POLAR can be found in Suarez Garcia (2010). However, the fluctuations in the background could be properly fitted using the 3ML software. FermiGBM detected this GRB (Hamburg et al. 2016), and therefore spectral data from Fermi-GBM were used in the analysis. The joint spectral fit for this GRB can be seen in Fig. D.1. The effective area correction (applied to the POLAR data) found in the analysis was $1.09 \pm 0.02$. The polarization response of POLAR was produced using the IPN location provided for this GRB: RA $(J 2000)=0.888^{\circ}, \operatorname{Dec}(J 2000)=-14.700^{\circ}($ Svinkin et al. 2016 $)$, a localization error of $4^{\circ}$ was assumed in the response. It should be noted that the error presented in Svinkin et al. (2016) is very asymmetric, but because of the methods used in the analysis here, this cannot be taken properly into account as described earlier in this paper, and an average of the error is assumed. The GRB occurred far off-axis for POLAR at a $\theta$ angle of $80.5^{\circ}$ degrees. As a result, the sensitivity of POLAR is reduced for specific polarization angles (at $45^{\circ}$ and $135^{\circ}$ as explained in the section regarding GRB 170207A) as is clear in the posterior distributions. The posterior distributions of the spectral and polarization parameters are shown in Fig. D.2. The posterior distribution of the polarization parameters is shown in Fig. D. 3 and the measured scattering angle distribution superimposed by the posterior model predictions (blue) in Fig. D.3. The scattering angle distribution clearly indicates the large off-axis angle resulting in the $360^{\circ}$ modulation in the distribution. A PD of $13.0_{-13.0}^{+28.0 \%}$ is found which is compatible with an unpolarized flux, especially when considering the lack of sensitivity in the PA range where the fit converges. No constraining 99\% upper limit is found. Although it appears that the posterior distribution is dominated by the lack of sensitivity, the results do clearly exclude high PDs at any polarization angles away from $135^{\circ}$.

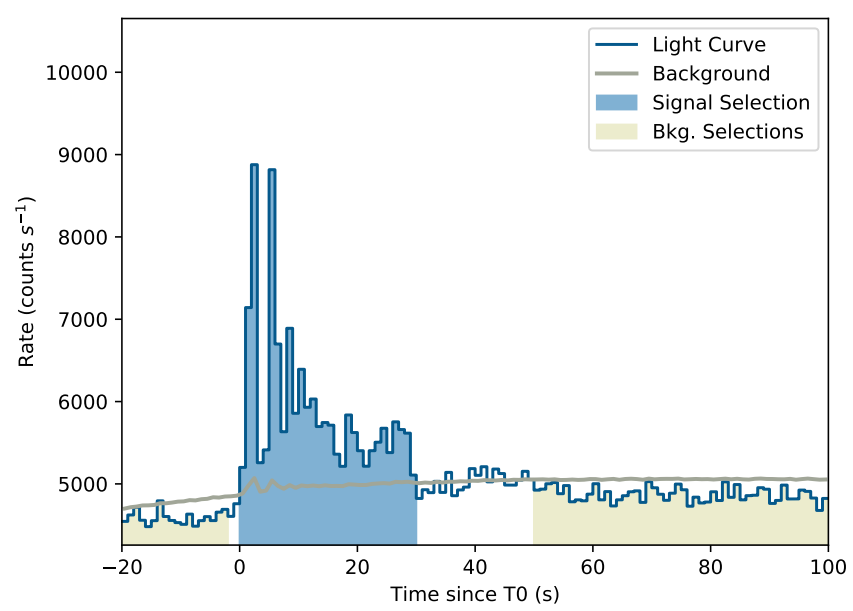

(a)

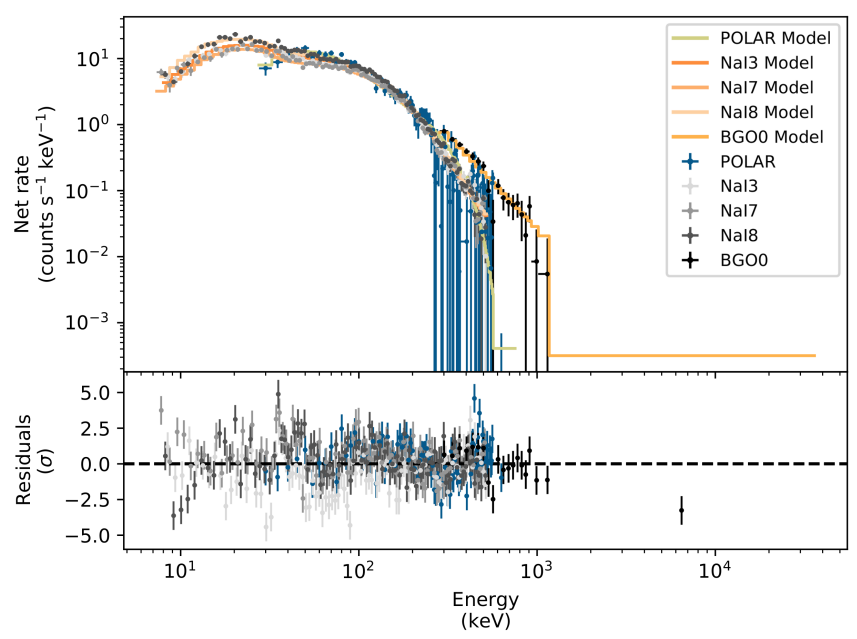

(b)

Fig. D.1. Panel a: light curve of GRB $161218 B$ as measured by POLAR, where $T=0 \mathrm{~s}$ is defined as the $T 0$ used by Fermi-GBM (Hamburg et al. 2016) for this GRB. Panel $b$ : joint spectral fit result for 161218B. The number of counts as detected by both POLAR (blue) and the different $\mathrm{NaI}$ and BGO detectors of Fermi-GBM (gray tints) are shown along with the best-fitting spectrum folded through the instrument responses in yellow for POLAR data and in orange tints for the Fermi-GBM data. The residuals for both data sets are shown at the bottom of the figure. 

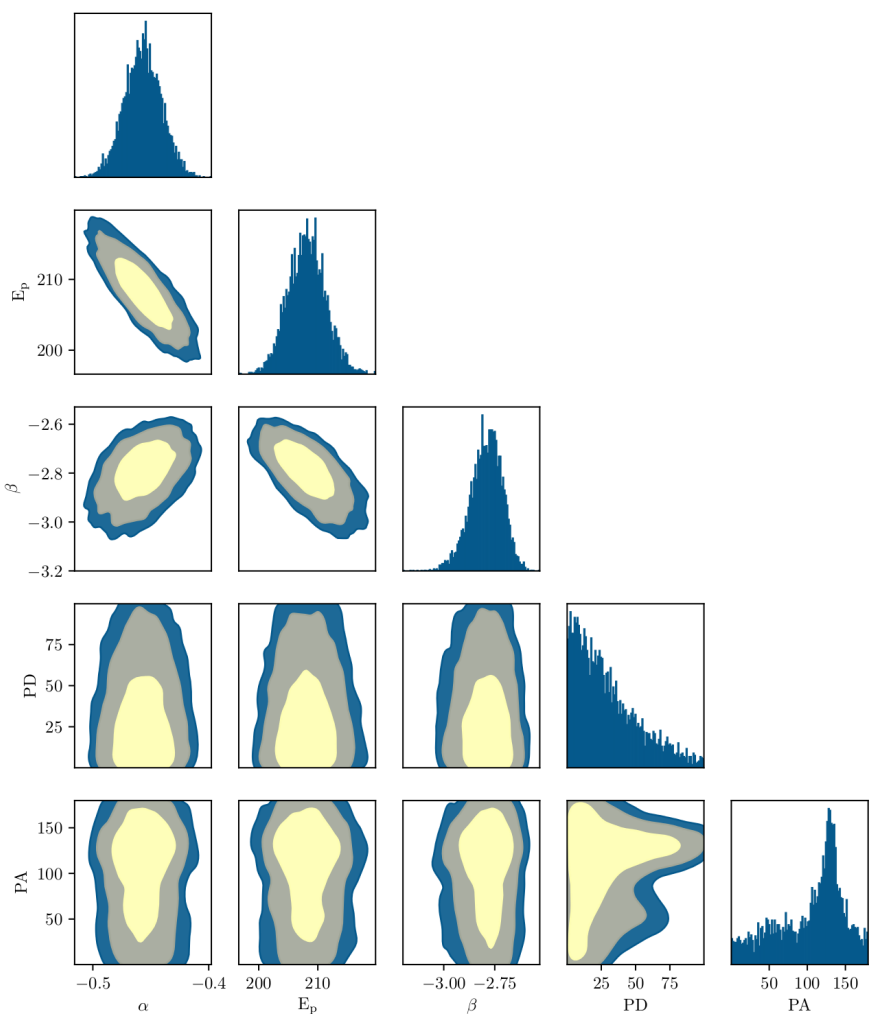

Fig. D.2. Spectral and polarization posterior distributions for GRB $161218 \mathrm{~B}$. The $1 \sigma$ and $2 \sigma$ credibility intervals as well as that corresponding to $99 \%$ are indicated. The polarization angle shown here is in the POLAR coordinate system; a rotation in the positive direction of 120 degrees transforms this to the coordinate system as defined by the IAU.
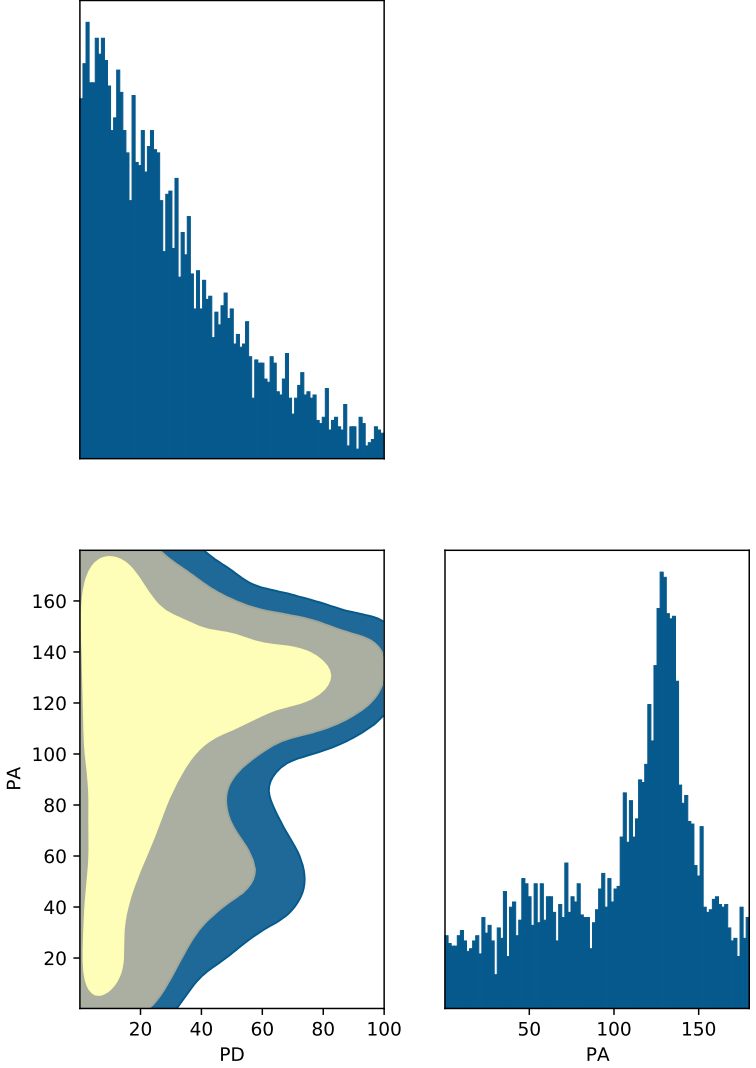

(a)

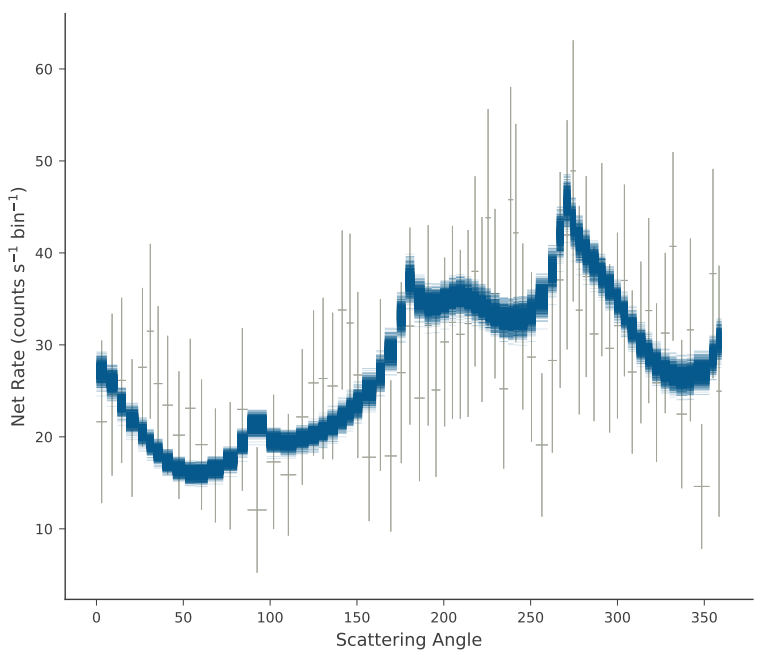

(b)

Fig. D.3. Panel a: polarization posterior distributions for GRB 161218B with the 1 and $2 \sigma$ credibility intervals as well as that corresponding to $99 \%$ credibility. The posterior distribution clearly reflects the lack of sensitivity for polarization angles of $135^{\circ}$ in this measurement. The polarization angle shown here is in the POLAR coordinate system; a rotation in the positive direction of 120 degrees transforms this to the coordinate system as defined by the IAU. Panel $b$ : measured scattering angle distribution (gray data points with a 5 degree bin size) with the posterior model predictions superimposed in blue. The errors on the data points are the Poisson errors corrected for the background. 


\section{Appendix E: 161229A}

The GRB 161229A was detected by POLAR and by FermiGBM. The latter define $T 0$ as December 29, 2016 at 21:03:48.82 (UT) (Fermi Collaboration 2016), which, for convenience is taken as $T 0$ for the analysis presented here. A $T_{90}$ of $(31.26 \pm$ 0.44) s was measured using POLAR data. The light curve, including the signal region (blue) and part of the background region (yellow) can be seen on the left in Fig. E.1. The GRB was detected by Fermi-GBM (Fermi Collaboration 2016) as trigger number 504738232. Data from Fermi-GBM were therefore used in the analysis. The spectral results from the joint fit for this GRB can be seen in Fig. E.1. The effective area correction (applied to the POLAR data) found in the analysis is $0.94 \pm 0.02$. The polarization response of POLAR was produced using the location calculated using the BALROG method, detailed in Berlato et al. (2019) using Fermi-GBM data for this GRB: RA $(\mathrm{J} 2000)=78.9^{\circ}$, Dec $(\mathrm{J} 2000)=6.2^{\circ}$, and a localization error of $2^{\circ}$ was assumed in the response. Using this location it is found that this GRB occurred far off-axis for POLAR at a $\theta$ angle of $87.6^{\circ}$ degrees. As a result, POLAR is almost fully insensitive for specific polarization angles (as described in the section regarding GRB 170207A) while it remains unaffected for other polarization angles as is clear in the posterior distributions. The posterior distributions of the spectral and polarization parameters are shown in Fig. E.2. Finally, the posterior distribution of the polarization parameters is shown together with measured scattering angle distribution superimposed by the posterior model predictions (blue) in Fig. E.3. A PD of $17_{-13}^{+24} \%$ is found which is compatible with an unpolarized flux especially when considering the lack of sensitivity in the polarization space for the polarization angle range where the fit converges. A $99 \%$ credibility upper limit for PD of $81.0 \%$ is found.

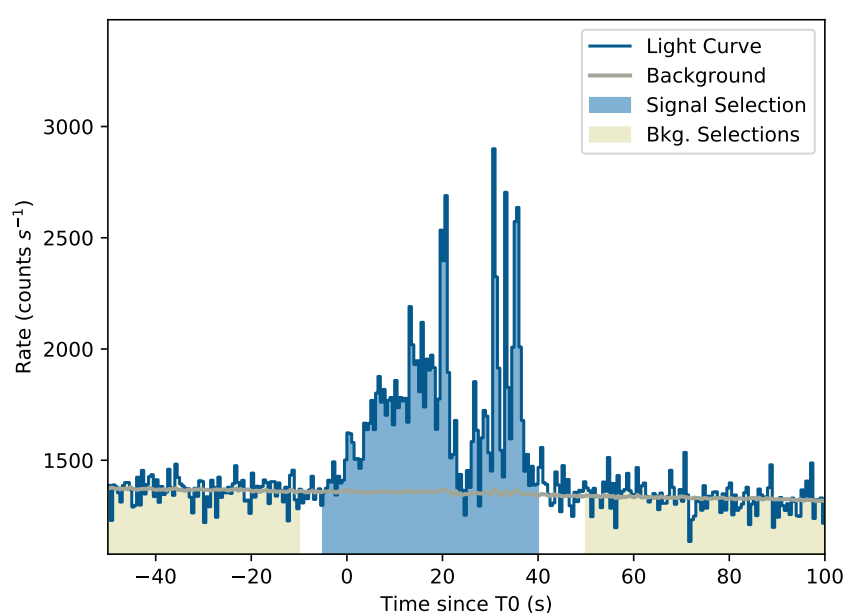

(a)

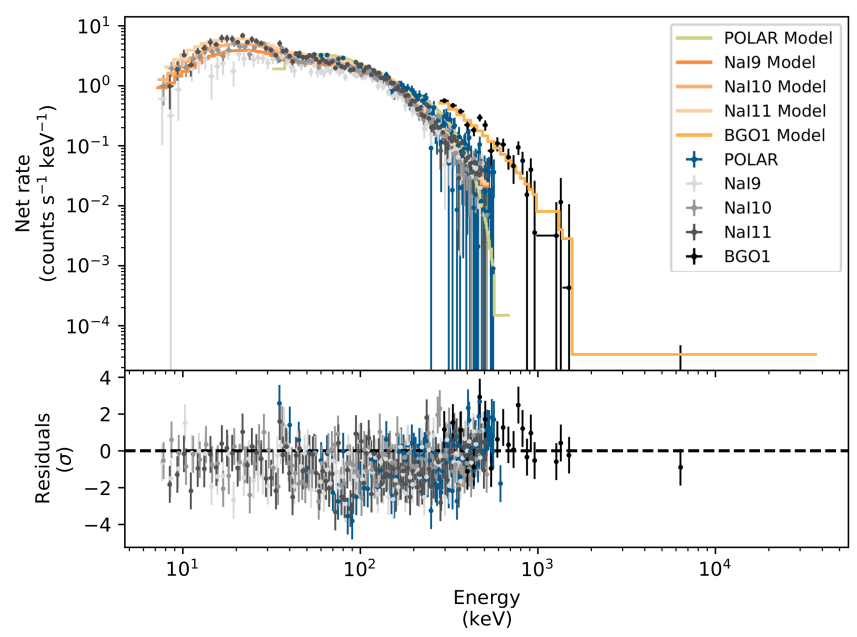

(b)

Fig. E.1. Panel a: light curve of GRB 161229A as measured by POLAR, where $T=0 \mathrm{~s}$ is defined as the $T 0$ employed by Fermi-GBM in their data products for this GRB (Fermi Collaboration 2016). Panel b: joint spectral fit result for 161229A. The number of counts as detected by both POLAR (blue) and the different NaI and BGO detectors of FermiGBM (gray tints) are shown along with the best fitting spectrum folded through the instrument responses in yellow for POLAR data and in orange tints for the Fermi-GBM data. The residuals for both data sets are shown at the bottom of the figure. 

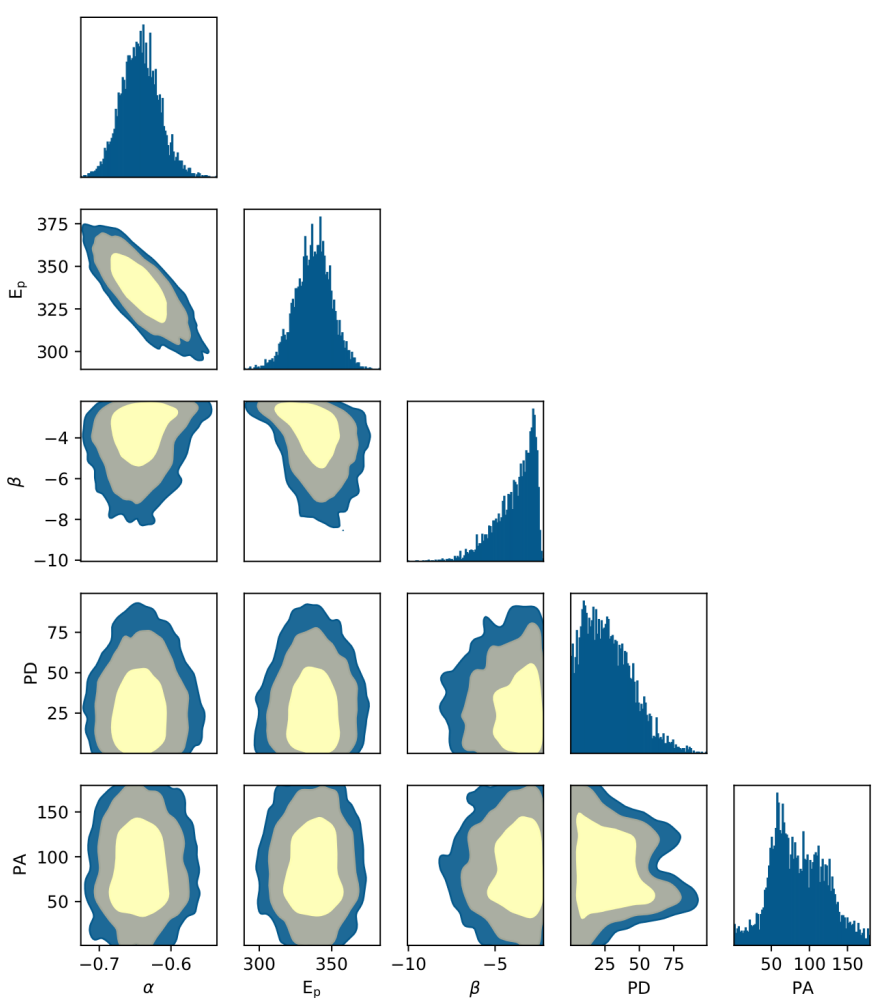

Fig. E.2. Spectral and polarization posterior distributions for GRB 161229A. The $1 \sigma$ and $2 \sigma$ credibility intervals as well as that corresponding to $99 \%$ are indicated. The polarization angle shown here is in the POLAR coordinate system; a rotation in the positive direction of 40 degrees transforms this to the coordinate system as defined by the IAU.
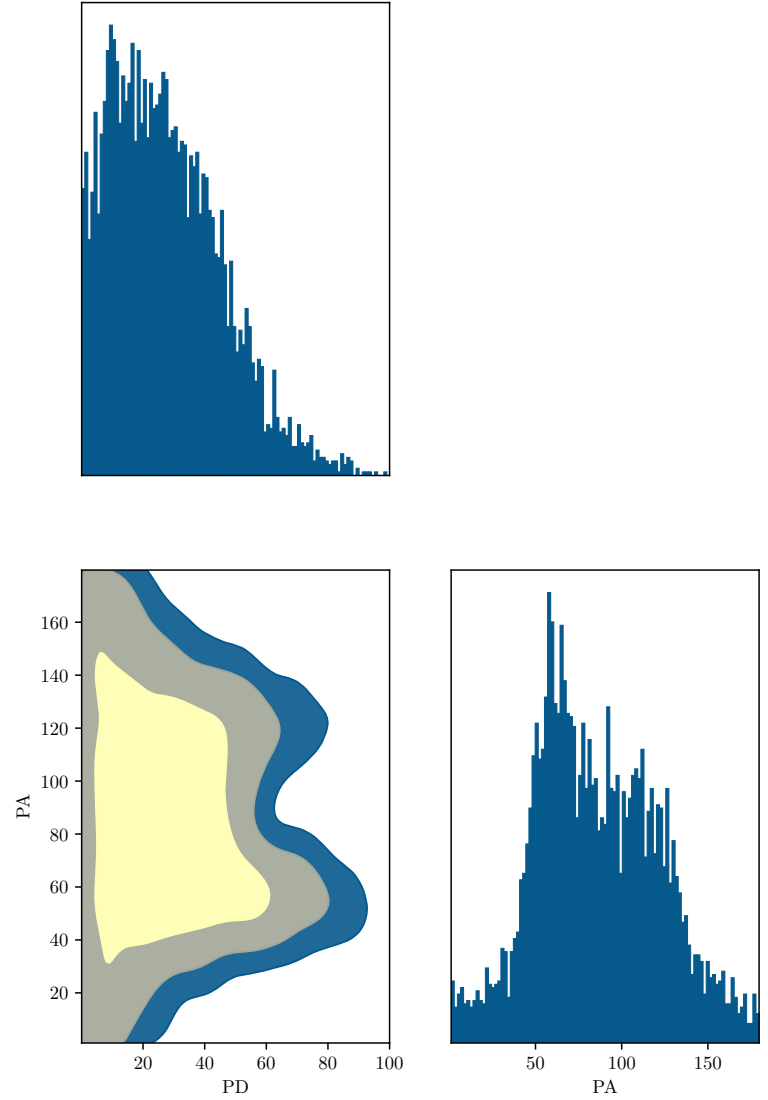

(a)

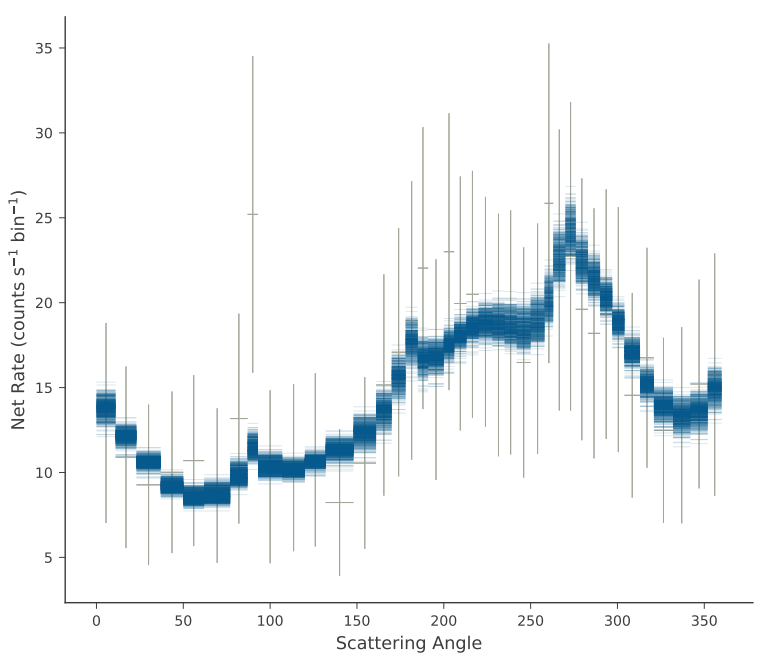

(b)

Fig. E.3. Panel a: polarization posterior distributions for GRB 161229A with the 1 and $2 \sigma$ credibility intervals as well as that corresponding to $99 \%$. The posterior distribution clearly reflects the lack of sensitivity for polarization angles of $135^{\circ}$ in this measurement. The polarization angle shown here is in the POLAR coordinate system; a rotation in the positive direction of 40 degrees transforms this to the coordinate system as defined by the IAU. Panel $b$ : measured scattering angle distribution (gray data points with a 9 degree bin size) with the posterior model predictions superimposed in blue. The errors on the data points are the Poisson errors corrected for the background. 


\section{Appendix F: 170101B}

The GRB 170101B, a long GRB with several overlapping pulses, was detected by POLAR and by Fermi-GBM. The latter reported a $T 0$ of January 1, 2017 at 02:47:17.87 (UT) (Veres et al. 2017a), which is used as the $T 0$ for the analysis presented here as well for convenience. A $T_{90}$ of $(11.14 \pm 0.38)$ s was measured using POLAR data. The light curve, including the signal region (blue) and part of the background region (yellow) can be seen on the left in Fig. F.1. Data from Fermi-GBM were used in the analysis. The spectral results of the joint fit for this GRB using both POLAR and Fermi-GBM data can be seen in Fig. F.1. The effective area correction (applied to the POLAR data) found in the analysis is $1.19 \pm 0.04$. The spectral fits for both instruments can be seen to agree well. The polarization response of POLAR was produced using the location calculated using the BALROG method described in Berlato et al. (2019) with FermiGBM data. The best-fitting location was: RA $(\mathrm{J} 2000)=69.6^{\circ}$, $\operatorname{Dec}(\mathrm{J} 2000)=-1.0^{\circ}$, and a localization error of $2^{\circ}$ was assumed. Using this location it is found that this GRB occurred relatively far off-axis for POLAR at a $\theta$ angle of $75.0^{\circ}$ degrees. As a result, POLAR is not very sensitive for specific polarization angles as described in the section describing the analysis of GRB 170207A. The posterior distributions of the spectral and polarization parameters are shown in Fig. F.2. Finally, the posterior distribution of the polarization parameters is shown together with the measured scattering angle distribution superimposed by the posterior model predictions (blue) in Fig. F.3. The scattering angle distributions clearly indicate the large off-axis angle resulting in the $360^{\circ}$ modulation in the distribution. A PD of $60_{-36}^{+24 \%}$ is found which, although not being fully incompatible with an unpolarized flux, suggests polarization. It should additionally be noted that the best-fitting PA of $76_{-21}^{+34^{\circ}}$ (as measured in the POLAR coordinate system) is largely unaffected by the above-mentioned loss of sensitivity due to the off-axis angle. The best-fitting PD is therefore likely not a result of this effect.

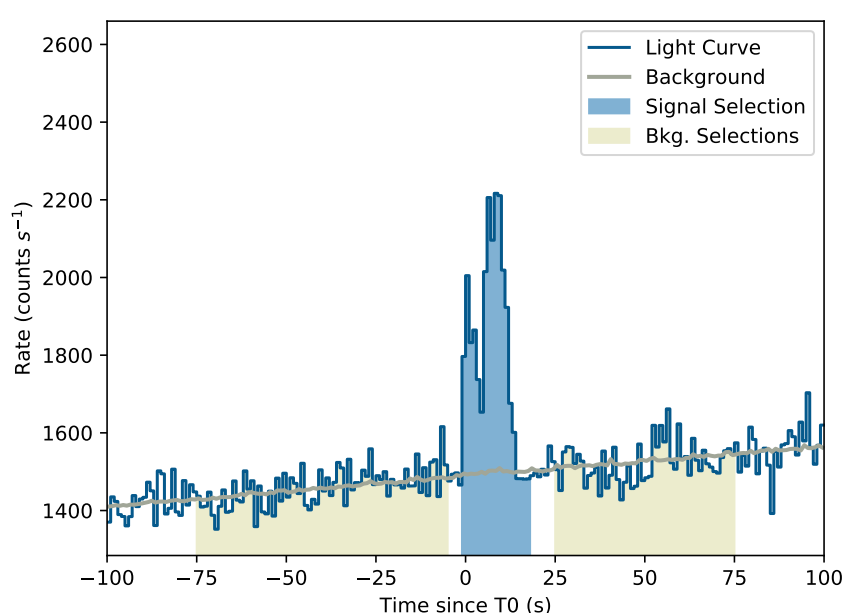

(a)

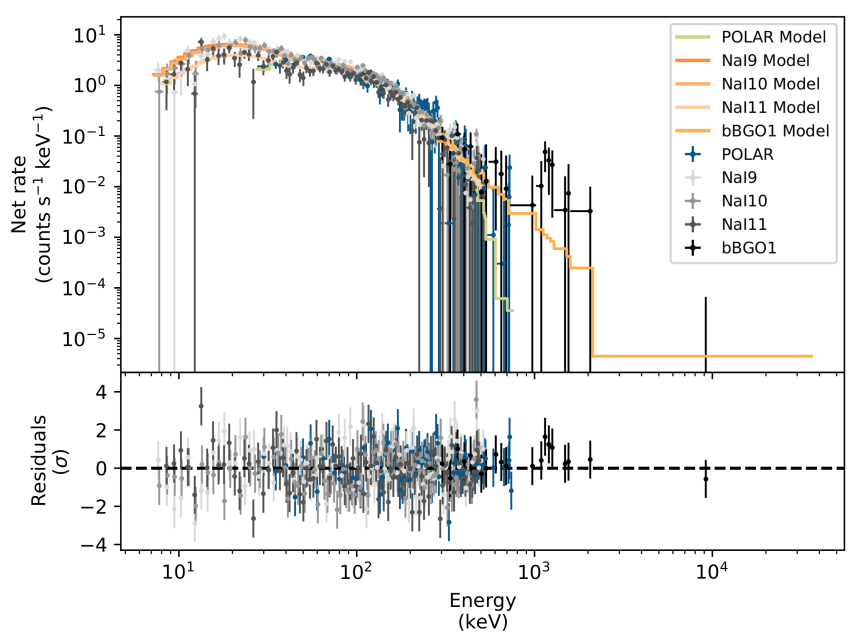

(b)

Fig. F.1. Panel a: light curve of GRB 170101B as measured by POLAR, where $T=0 \mathrm{~s}$ is defined as the $T 0$ employed by Fermi-GBM in their data products for this GRB (Veres et al. 2017a). Panel $b$ : joint spectral fit result for 170101B. The number of counts as detected by both POLAR (blue) and the different NaI and BGO detectors of Fermi-GBM (gray tints) are shown along with the best-fitting spectrum folded through the instrument responses in yellow for POLAR data and in orange tints for the Fermi-GBM data. The residuals for both data sets are shown at the bottom of the figure. 

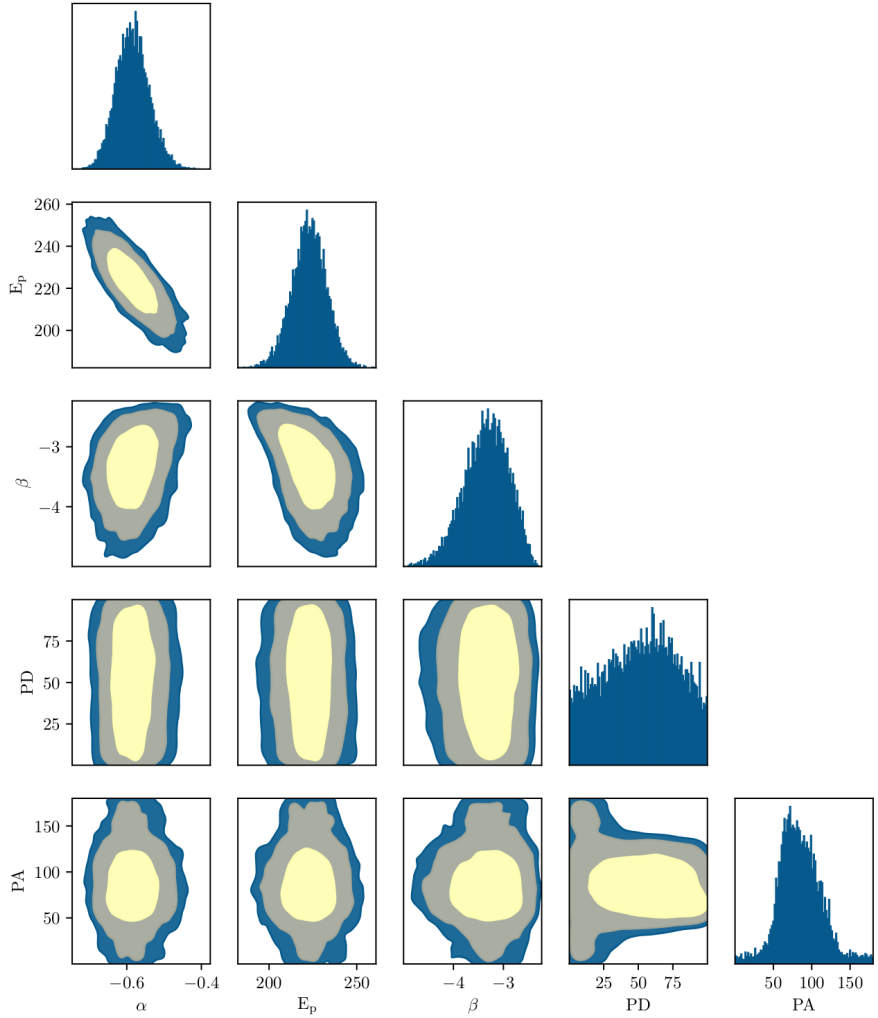

Fig. F.2. Spectral and polarization posterior distributions for GRB $170101 \mathrm{~B}$. The 1 and $2 \sigma$ credibility intervals as well as that corresponding to $99 \%$ are indicated. The polarization angle shown here is in the POLAR coordinate system; a rotation in the positive direction of 33 degrees transforms this to the coordinate system as defined by the IAU.
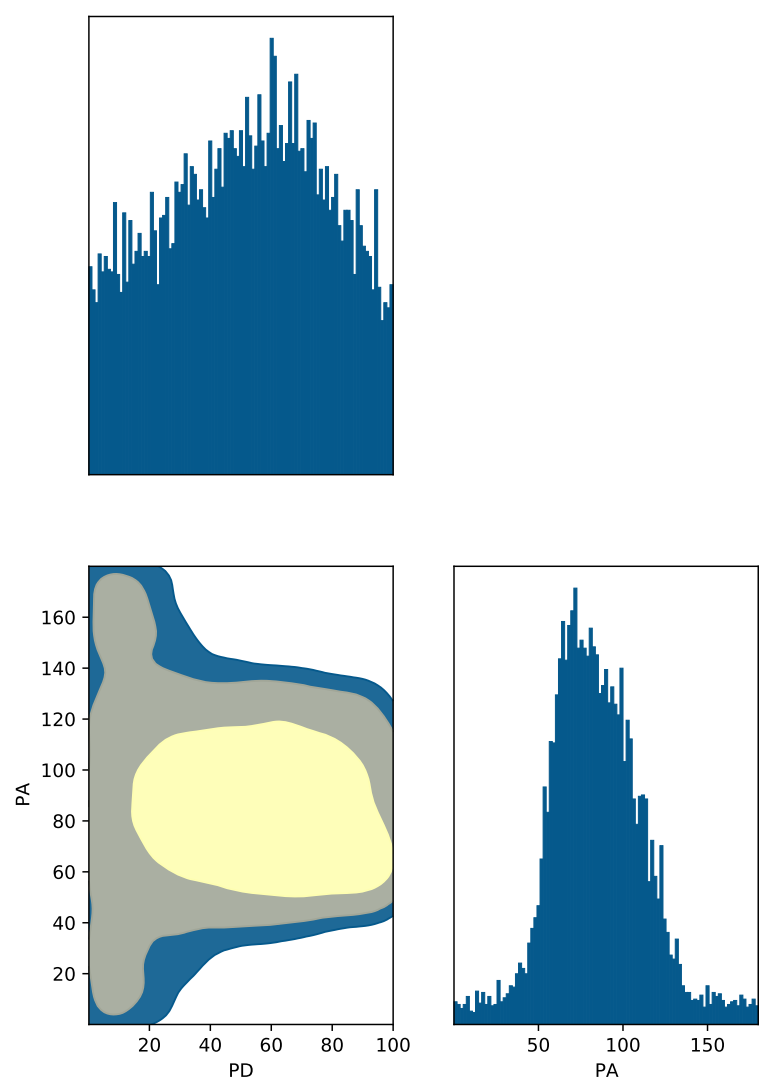

(a)

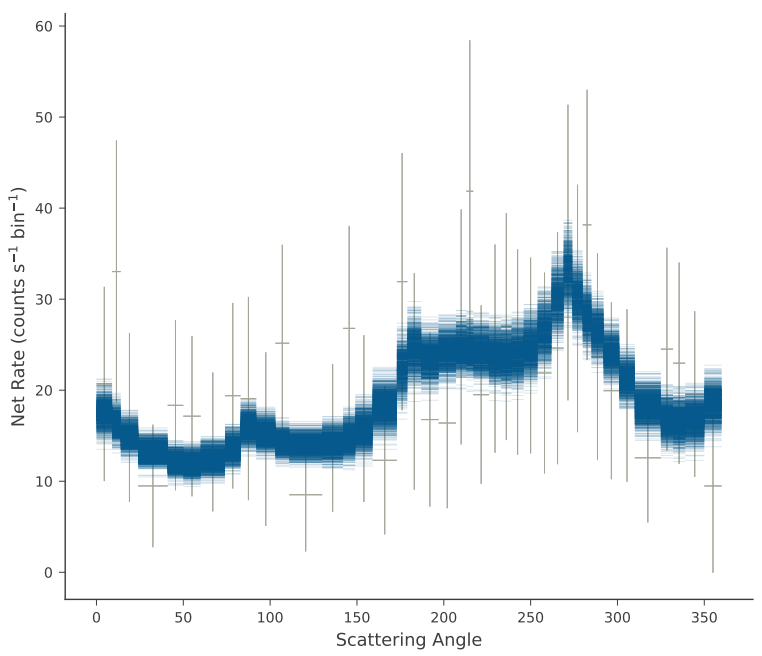

(b)

Fig. F.3. Panel a: polarization posterior distributions for GRB 170101B with the 1 and $2 \sigma$ credibility intervals as well as that corresponding to 99\% credibility. The polarization angle shown here is in the POLAR coordinate system; a rotation in the positive direction of 33 degrees transforms transforms this to the coordinate system as defined by the IAU. Panel $b$ : measured scattering angle distribution (gray data points with a 9 degree bin size) with the posterior model predictions superimposed in blue. The errors on the data points are the Poisson errors corrected for the background. 


\section{Appendix G: 170114A}

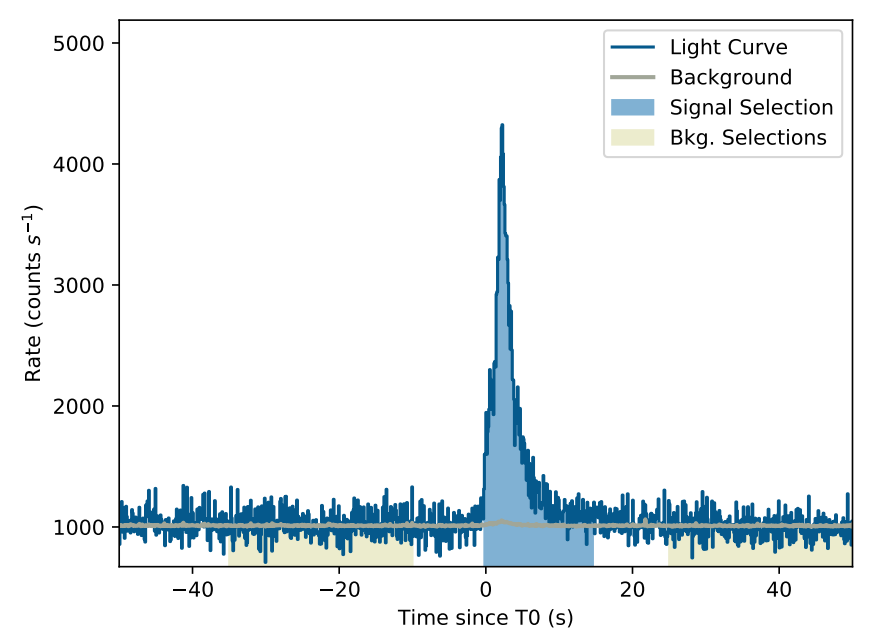

(a)

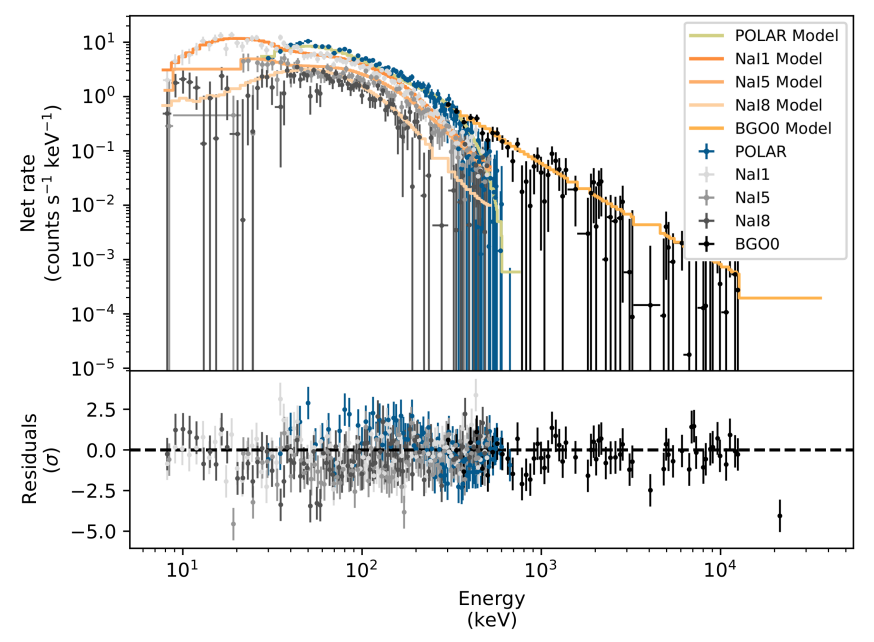

(b)

Fig. G.1. Panel a: light curve of GRB $170114 \mathrm{~A}$ as measured by POLAR where $T=0 \mathrm{~s}$ is defined as the $T 0$ employed by Fermi-GBM (Veres et al. 2017b) for this GRB. Panel b: joint spectral fit result for 170114A. The number of counts as detected by both POLAR (blue) and the different NaI and BGO detectors of Fermi-GBM (gray tints) are shown along with the best-fitting spectrum folded through the instrument responses in yellow for POLAR data and in orange tints for the Fermi-GBM data. The residuals for both data sets are shown at the bottom of the figure.

The GRB 170114A was detected by POLAR and Fermi-GBM. The latter reported a $T 0$ of January 14, 2017 at 22:01:09.50 (UT) (Veres et al. 2017b) which is also taken as $T 0$ for the analysis presented here for convenience. A $T_{90}$ of $(10.48 \pm 0.16) \mathrm{s}$ was measured using POLAR data. The polarization properties of this GRB has been extensively studied in a previous publication (Burgess et al. 2019) using the same methodology as that used here. In this latter publication, a time-integrated analysis found a polarization consistent with an unpolarized flux, while a timeresolved analysis results in a PD of around $30 \%$ with a changing polarization angle after an initial unpolarized flux during the start of the GRB. Additionally, it was found that the spectrum of this GRB is consistent with that from synchrotron radiation. However, in this work we perform the analysis using a Band function

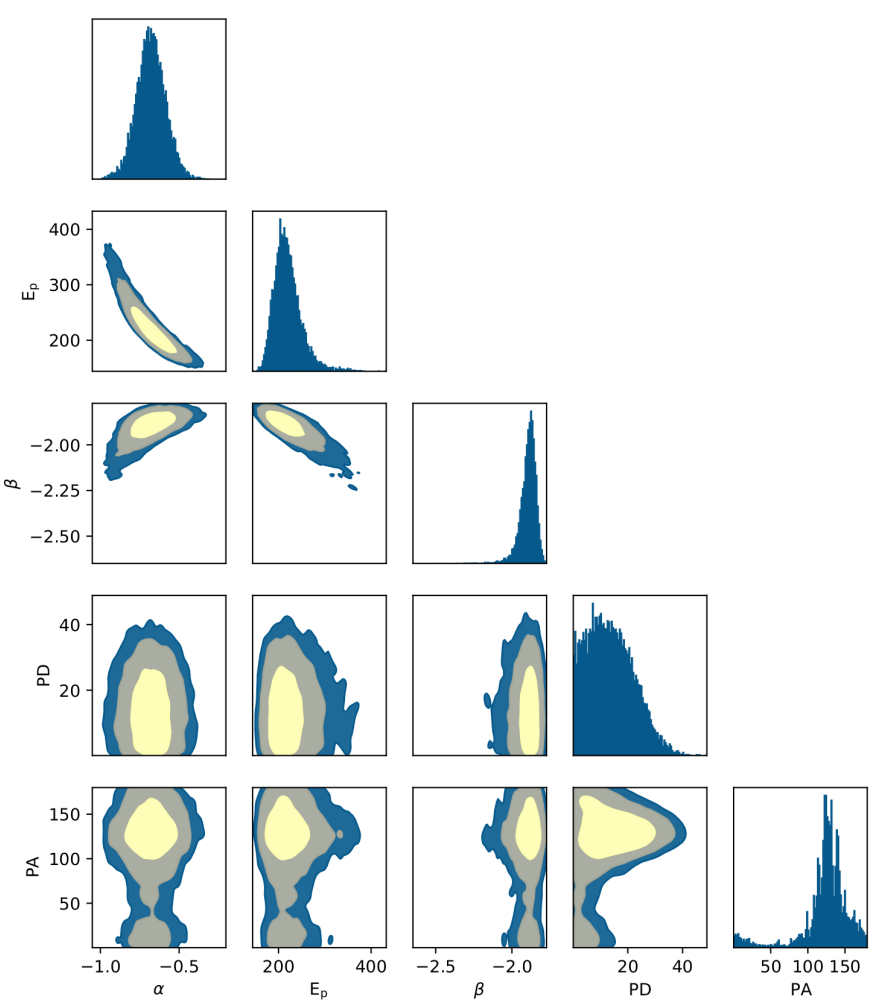

Fig. G.2. Spectral and polarization posterior distributions for GRB 170114A. The 1 and $2 \sigma$ credibility intervals as well as that corresponding to $99 \%$ are indicated. The polarization angle shown here is in the POLAR coordinate system; a rotation in the positive direction of 38 degrees transforms this to the coordinate system as defined by the IAU.

instead of a physical synchrotron model. We include only a summary of our results from the analysis here for completeness, and to show that consistent results are found when using the Band function instead of the more physical synchrotron model. For details on this GRB and its polarization characteristics we refer to reader to Burgess et al. (2019).

The light curve, including the signal region (blue) and the background region (yellow), can be seen in Fig. G.1. The GRB was detected by Fermi-GBM (Veres et al. 2017b) allowing spectral data from this instrument to be used in the analysis. The spectral results of the joint fit can be seen in Fig. G.1. The fit results of both instruments can be seen to be in good agreement for the full energy range. The effective area correction (applied to the POLAR data) found in the analysis was $1.24 \pm$ 0.04 . The polarization response of POLAR was produced using the location calculated using the BALROG method detailed in Berlato et al. (2019) using Fermi-GBM data for this GRB. The location of the GRB found using this method corresponds to: RA $(\mathrm{J} 2000)=13.10^{\circ}$, Dec $(\mathrm{J} 2000)=-13.0^{\circ}$, and a localization error of $2^{\circ}$ was assumed in the response. The posterior distributions of the spectral and polarization parameters are shown in Fig. G.2. Finally, the posterior distribution of the polarization parameters is shown together with measured scattering angle distribution superimposed by the posterior model predictions (blue) in Fig. G.3. A PD of $10.1_{-7.4}^{+10.5 \%}$ is found which is compatible with that reported by Zhang et al. (2019a), but it should be noted that the PA used by Zhang et al. (2019a) is presented following the IAU convention while here we measure it in the POLAR coordinate system; a rotation of 142 degrees in the negative direction is 

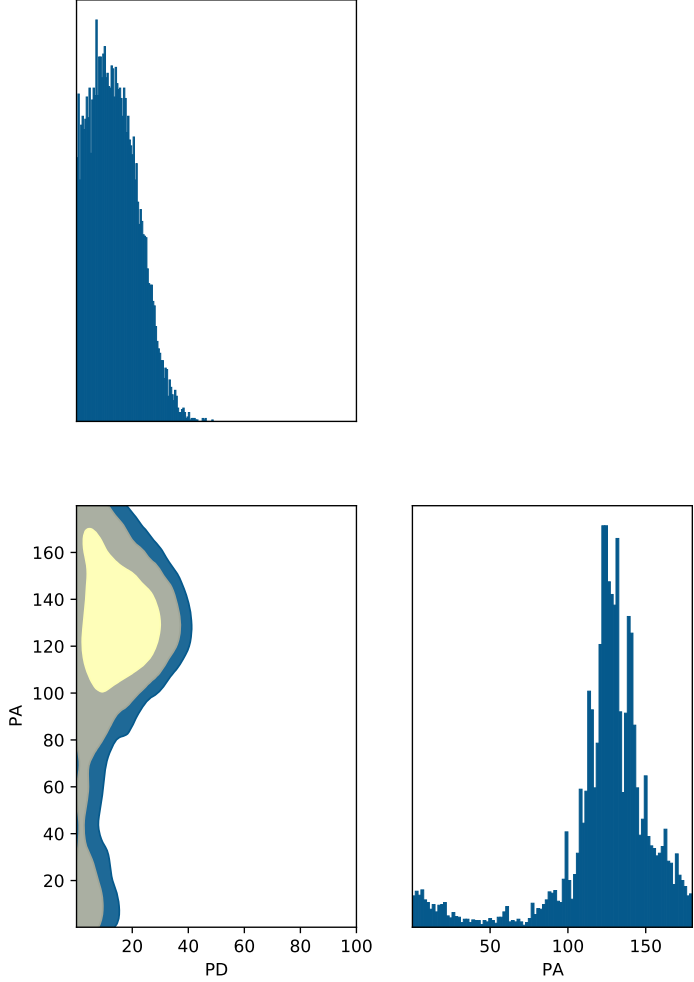

(a)

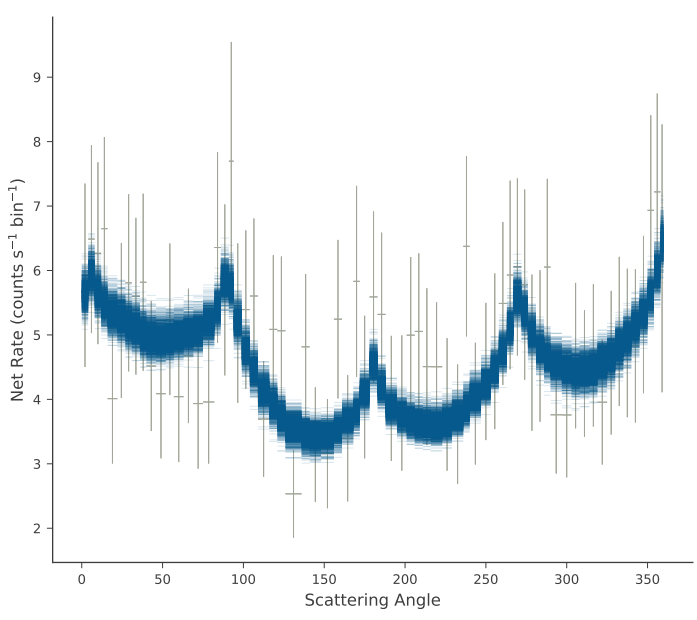

(b)

Fig. G.3. Panel a: polarization posterior distributions for GRB 170114A with the 1 and $2 \sigma$ credibility intervals as well as that corresponding to 99\%. The polarization angle shown here is in the POLAR coordinate system; a rotation in the positive direction of 38 degrees transforms this to the coordinate system as defined by the IAU. Panel $b$ : measured scattering angle distribution (gray data points with a 9 degree bin size) with the posterior model predictions superimposed in blue. The errors on the data points are the Poisson errors corrected for the background.

required to move to the coordinate system as defined by the IAU. A $99 \%$ credibility upper limit of $35.9 \%$ is found for this GRB.
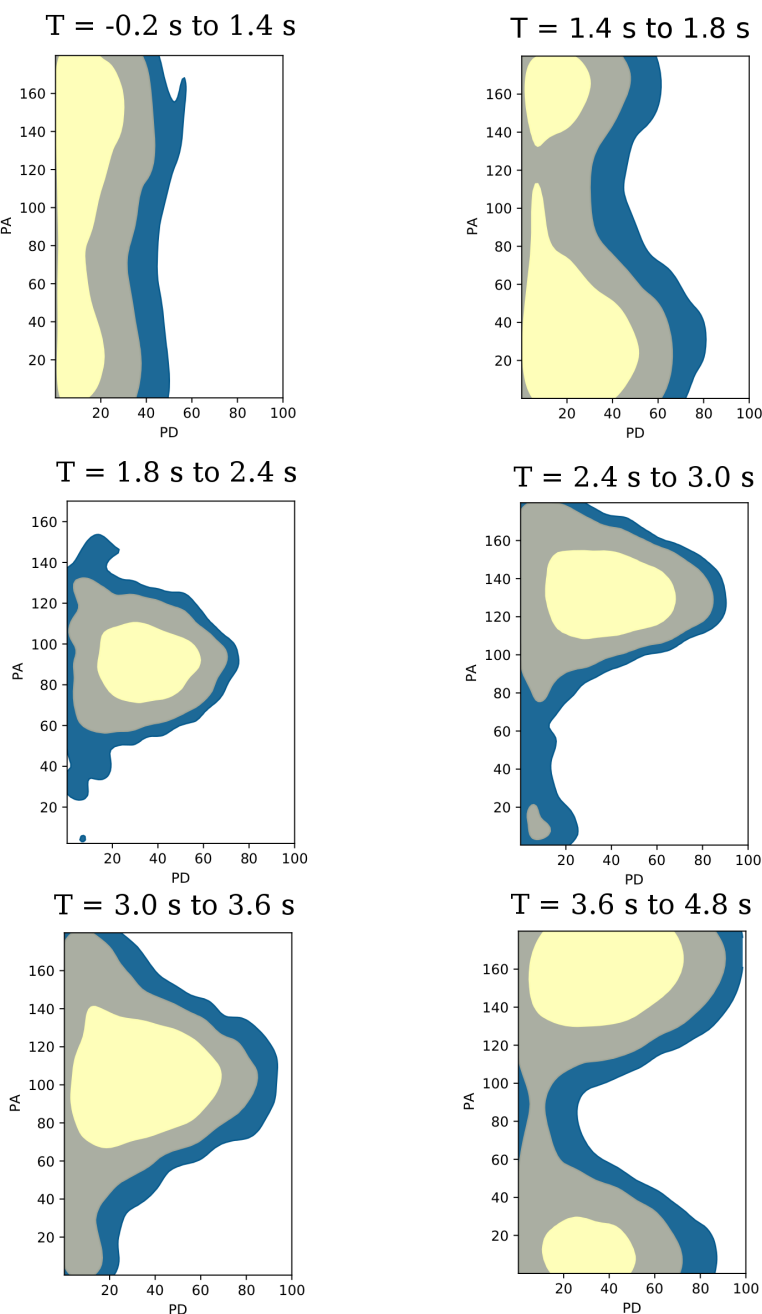

Fig. G.4. Polarization posterior distributions for the time bins of GRB 170114A are shown with the 1 and $2 \sigma$ credibility intervals as well as that corresponding to $99 \%$ credibility. The polarization angle shown here is in the POLAR coordinate system; a rotation (in the negative direction) of 142 degrees transforms this to the coordinate system as defined by the IAU.

In order to verify that the current analysis yields results consistent with those found by Burgess et al. (2019) for a timeresolved analysis, the same data from the time bins as those calculated using the method in Burgess et al. (2019) were studied here. In Fig. G.4 we present the posteriors for the polarization for the first six time bins, those corresponding to $T=-0.2-1.4 \mathrm{~s}$, $T=1.4-1.8 \mathrm{~s}, T=1.8-2.4 \mathrm{~s}, T=2.4-3.0 \mathrm{~s}, T=3.0-3.6 \mathrm{~s}$, and $T=3.6-4.8 \mathrm{~s}$. It was found that the polarization parameters found here are consistent with those found in Burgess et al. (2019). The results point to an initially unpolarized flux followed by four time bins with a PD of around $30 \%$ where the PA varies from time bin to time bin. The polarization angles shown here are in the POLAR coordinate system again. It can be concluded that the same polarization angles and changes are found between both analyses. 


\section{Appendix H: 170127C}

The GRB 170127C, a bright short GRB with several overlapping pulses, was detected by POLAR and by Fermi-GBM who report a $T 0$ of January 27, 2017 at 01:35:47.79 (UT) (Bissaldi et al. 2017). For convenience this will be used as the $T 0$ for the analysis presented here as well. A $T_{90}$ of $(0.14 \pm 0.01) \mathrm{s}$ was measured using POLAR data. The light curve, including the signal region (blue) and the background region (yellow) can be seen in Fig. H.1 along with a more detailed inset of the pulse region. It should be noted here that a large period of the time after the GRB was excluded as background, because a longer lasting afterglow was reported by Konus-Wind (Frederiks et al. 2017) for this GRB. This late emission is additionally visible in the POLAR data. A separate spectral fit was performed on this afterglow using both POLAR and Fermi-GBM data. The results indicate that the emission is significantly softer than the rather hard emission in the bright first peak. The spectral parameters for both periods are reported in Table 1. As we assume this afterglow to have a different origin based on the spectral results, we exclude it from the polarization analysis here. Data from Fermi-GBM were used for the joint analysis. The spectral results of the joint fit can be seen in Fig. H.1. The effective area correction (applied to the POLAR data) found in the analysis is $0.60 \pm 0.23$. The response was produced using the location provided by FermiLAT: RA $(J 2000)=339.3^{\circ}$, Dec $(J 2000)=-63.9^{\circ}$ (Moretti et al. 2017). The posterior distributions of the spectral and polarization parameters are shown in Fig. H.2. Finally, the posterior distribution of the polarization parameters is shown together with measured scattering angle distribution superimposed by the posterior model predictions (blue) in Fig. H.3. A PD of $9.9_{-9.9}^{+19.3 \%}$ is found which is compatible with that reported by Zhang et al. (2019a). It should be noted that the PA used by Zhang et al. (2019a) is measured in the IAU coordinate system while here we measure it in the POLAR coordinate system. A rotation of $50^{\circ}$ in the negative direction transforms the POLAR coordinate system to that as defined by the IAU for this GRB. A $99 \%$ credibility upper limit for PD of $61.2 \%$ is found.

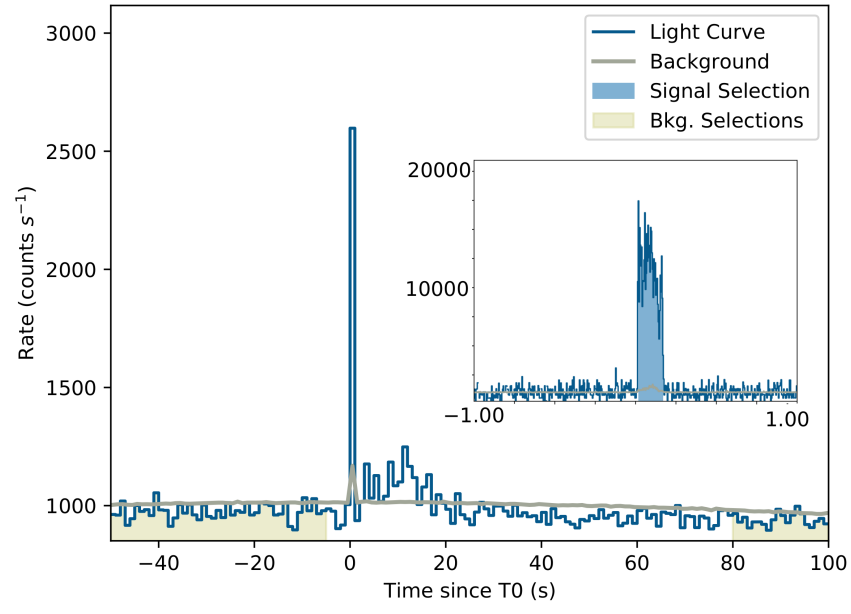

(a)

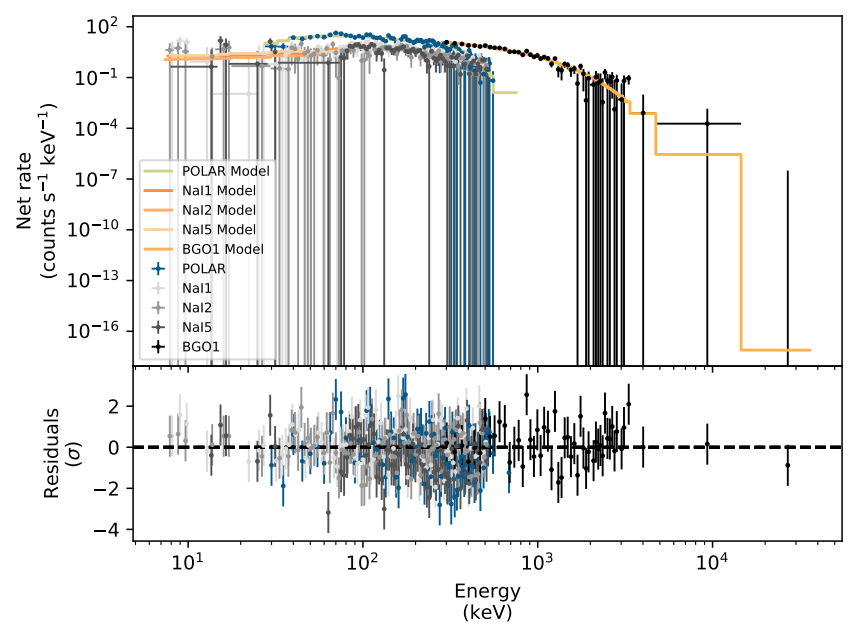

(b)

Fig. H.1. Panel $a$ : light curve of GRB $170127 \mathrm{C}$ as measured by POLAR with an inlay of a zoomed-in version around the peak, where $T=0 \mathrm{~s}$ is defined as the $T 0$ employed by Fermi-GBM in their data products for this GRB (Bissaldi et al. 2017). The spectrum of the late emission, which can be seen until approximately $20 \mathrm{~s}$, was fitted separately. The results of this are reported in Table 1 . Panel $b$ : joint spectral fit result for GRB 170127C. The number of counts as detected by both POLAR (blue) and the different $\mathrm{NaI}$ and BGO detectors of Fermi-GBM (gray tints) are shown along with the best-fitting spectrum folded through the instrument responses in yellow for POLAR data and in orange tints for the Fermi-GBM data. The residuals for both data sets are shown at the bottom of the figure. 


\section{Kole et al.: The POLAR gamma-ray burst polarization catalog}
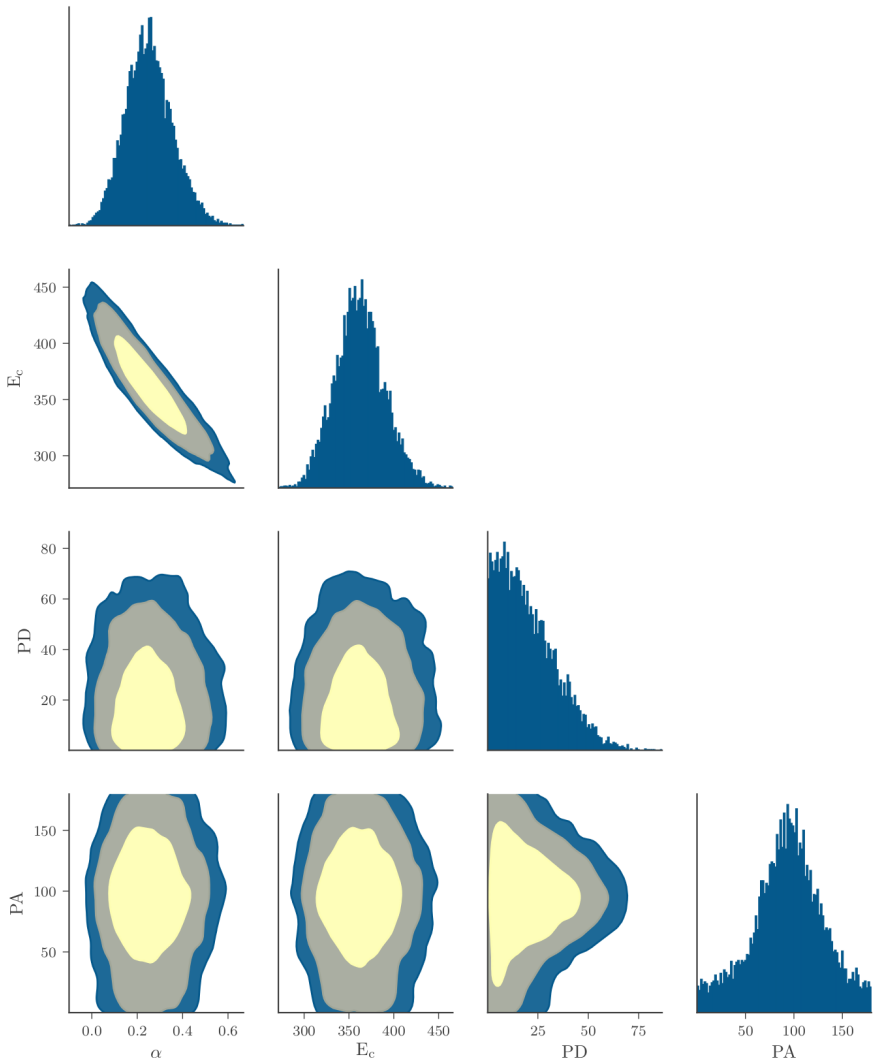

Fig. H.2. Spectral and polarization posterior distributions for GRB $170127 \mathrm{C}$. The 1 and $2 \sigma$ credibility intervals as well as that corresponding to $99 \%$ are indicated. The polarization angle shown here is in the POLAR coordinate system, a rotation in the positive direction of 130 degrees transforms transforms this to the coordinate system as defined by the IAU.
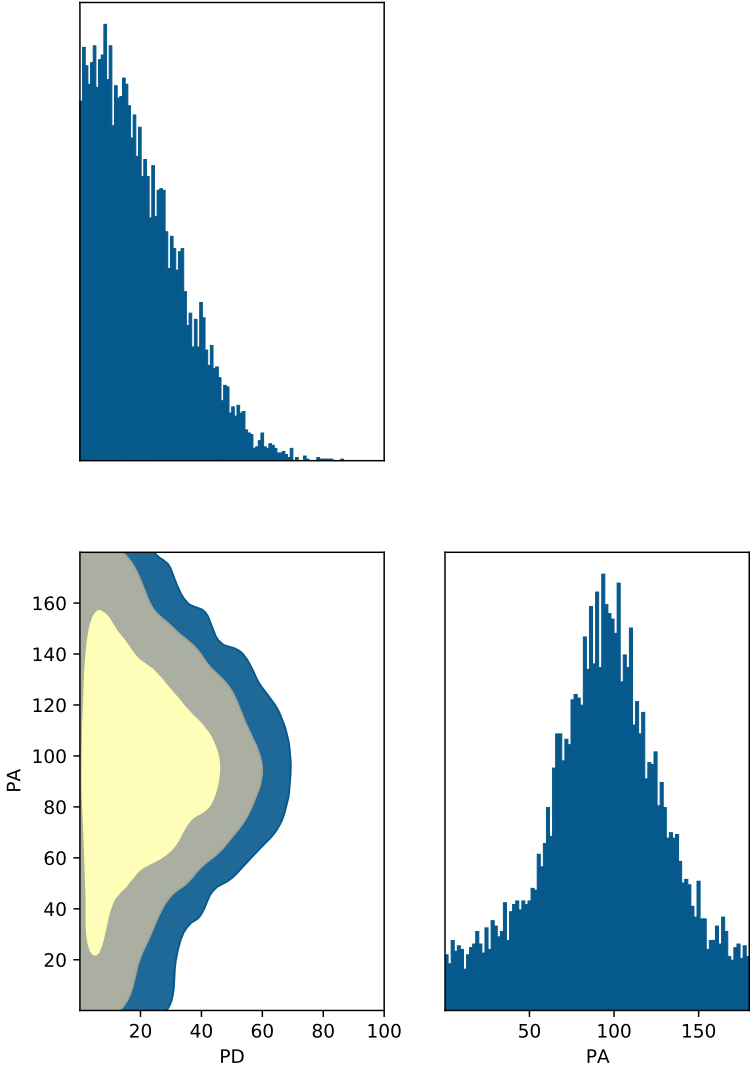

(a)

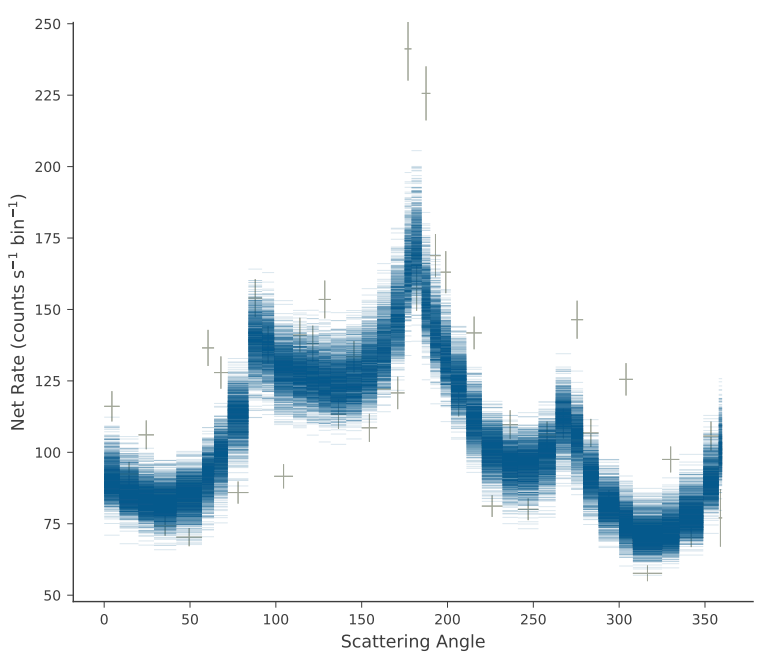

(b)

Fig. H.3. Panel a: polarization posterior distributions for GRB 170127C with the 1 and $2 \sigma$ credibility intervals as well as that corresponding to $99 \%$ credibility. The polarization angle shown here is in the POLAR coordinate system, a rotation in the positive direction of 130 degrees transforms this to the coordinate system as defined by the IAU. Panel $b$ : measured scattering angle distribution (gray data points with a 9 degree bin size) with the posterior model predictions superimposed in blue. The errors on the data points are the Poisson errors corrected for the background. 


\section{Appendix I: 170206A}

The GRB 170206A, a bright multi-pulse GRB was detected by POLAR and by Fermi-GBM who report a $T 0$ of February 6, 2017 at 10:51:57.70 (UT) (von Kienlin et al. 2017). For convenience, this is taken as $T 0$ for the analysis presented here as well. A $T_{90}$ of $(1.26 \pm 0.01) \mathrm{s}$ was measured using POLAR data. The light curve, including the signal region (blue) and part of the background region (yellow) can be seen in Fig. I.1. As it was also detected by Fermi-GBM (von Kienlin et al. 2017) the spectral data from this instrument were used for the joint analysis. The spectral results of the joint fit results can be seen in Fig. I.1. The effective area correction (applied to the POLAR data) found in the analysis was $0.93 \pm 0.02$. The POLAR response was produced using the location provided by Fermi-LAT: RA $(J 2000)=212.79^{\circ}$, Dec $(J 2000)=14.48^{\circ}($ Fana Dirirsa et al. 2017). The posterior distributions of the spectral and polarization parameters are shown in Fig. I.2. Finally, the posterior distribution of the polarization parameters is shown together with measured scattering angle distribution superimposed by the posterior model predictions (blue) in Fig. I.3. A PD of $13.5_{-8.6}^{+7.4 \%}$ is found which is compatible with that reported by Zhang et al. (2019a). It should be noted that the PA used by Zhang et al. (2019a) is measured in the GRB coordinate system while here we measure it in the POLAR coordinate system. A $99 \%$ credibility upper limit for PD of $34.0 \%$ is found.

Additionally, time-resolved studies were performed for this GRB. The three pulses in the emission were studied independently. The first selected time bin is from $T=0.0 \mathrm{~s}$ to $T=0.8 \mathrm{~s}$, the second from $T=0.8 \mathrm{~s}$ to $T=1.2 \mathrm{~s}$ and the third from $T=1.2 \mathrm{~s}$ to $T=2.0 \mathrm{~s}$. The polarization of the three time intervals was found to be $8.9_{-7.3}^{+15.0}, 7.4_{-5.7}^{+13.9}$ and $14_{-10}^{+16}$ and therefore compatible with an unpolarized flux. The following three upper limits for PD were found for the three respective time intervals: $46.2 \%$, $40.0 \%$, and $56.0 \%$.

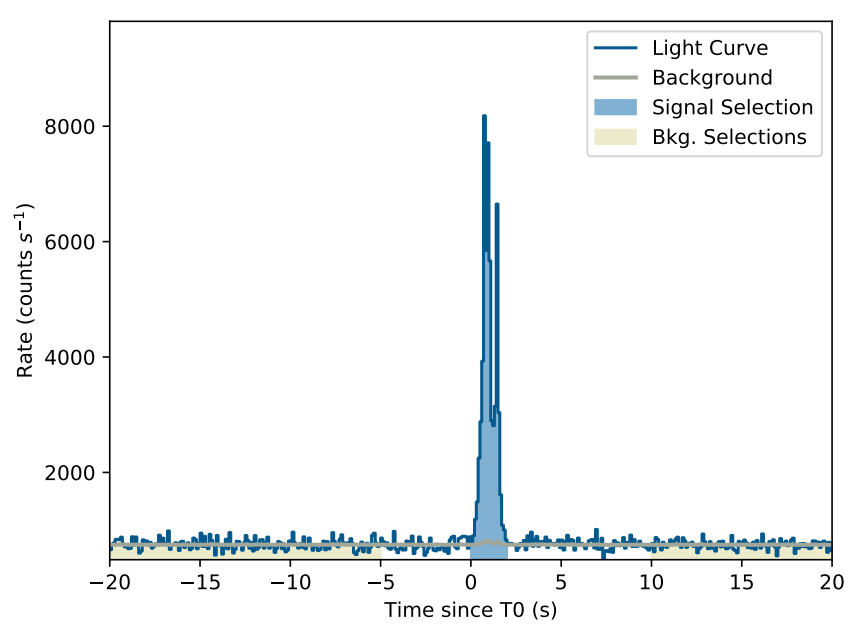

(a)

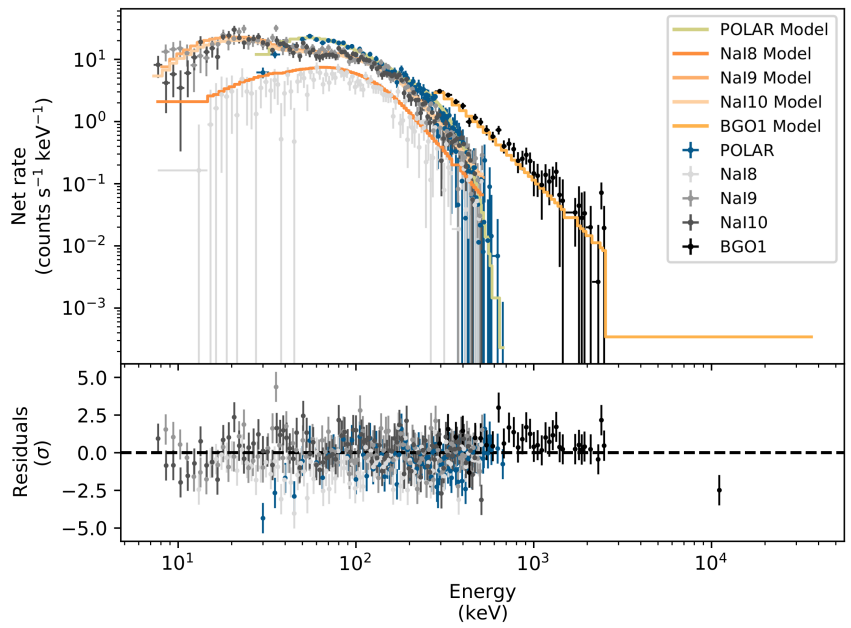

(b)

Fig. I.1. Panel $a$ : light curve of GRB 170206A as measured by POLAR, where $T=0 \mathrm{~s}$ is defined as the $T 0$ employed by Fermi-GBM in their data products (von Kienlin et al. 2017). Panel $b$ : joint spectral fit result for 170206A. The number of counts as detected by both POLAR (blue) and the different NaI and BGO detectors of Fermi-GBM (gray tints) are shown along with the best-fitting spectrum folded through the instrument responses in yellow for POLAR data and in orange tints for the Fermi-GBM data. The residuals for both data sets are shown at the bottom of the figure. 

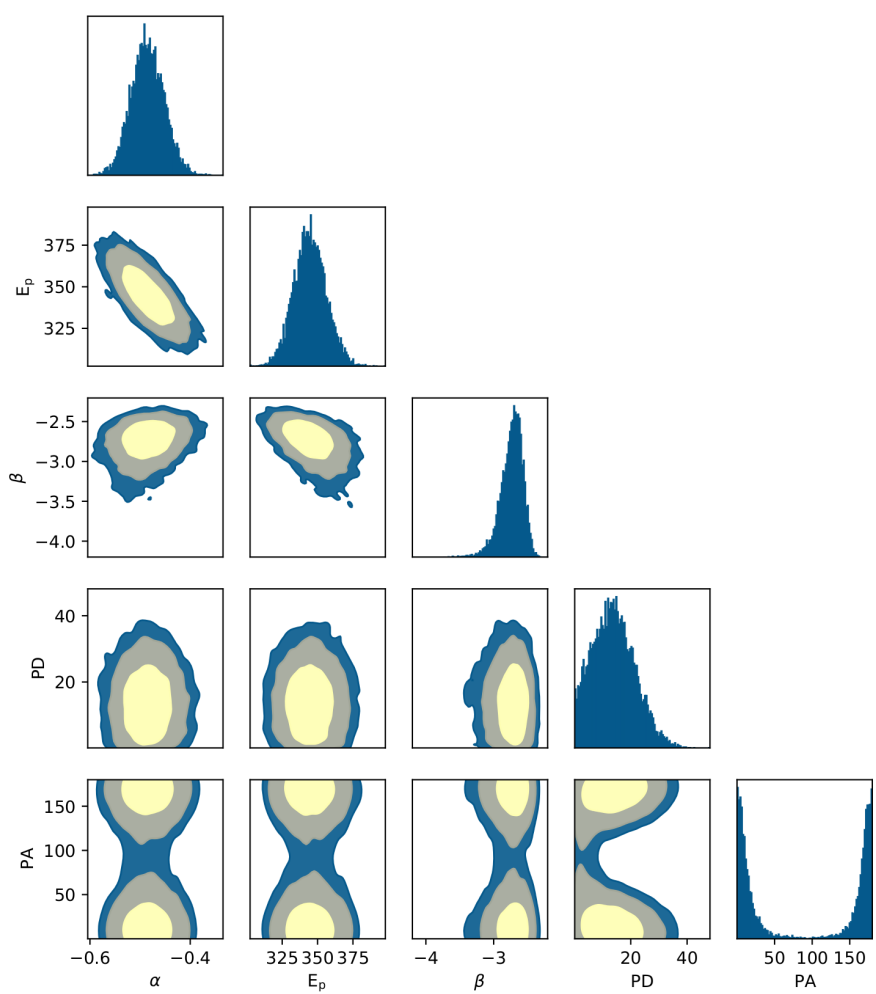

Fig. I.2. Spectral and polarization posterior distributions for GRB $170206 \mathrm{~A}$. The 1 and $2 \sigma$ credibility intervals as well as that corresponding to $99 \%$ are indicated. The polarization angle shown here is in the POLAR coordinate system; a rotation in the positive direction of 106 degrees transforms this to the coordinate system as defined by the IAU.
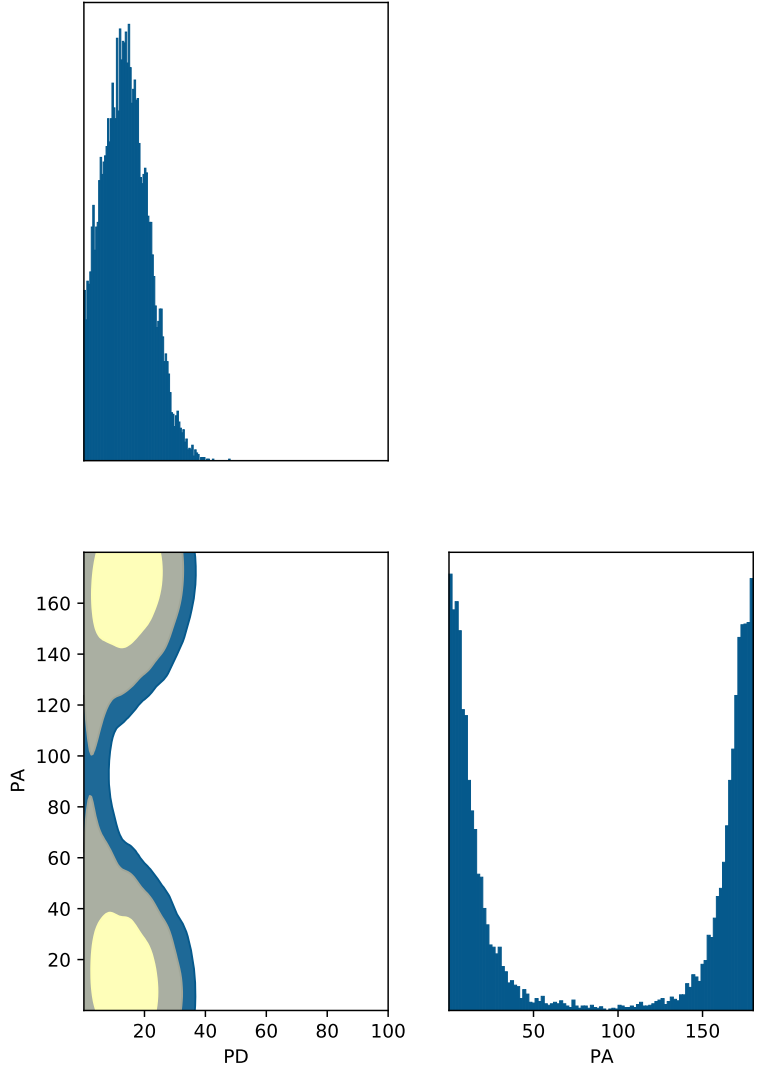

(a)

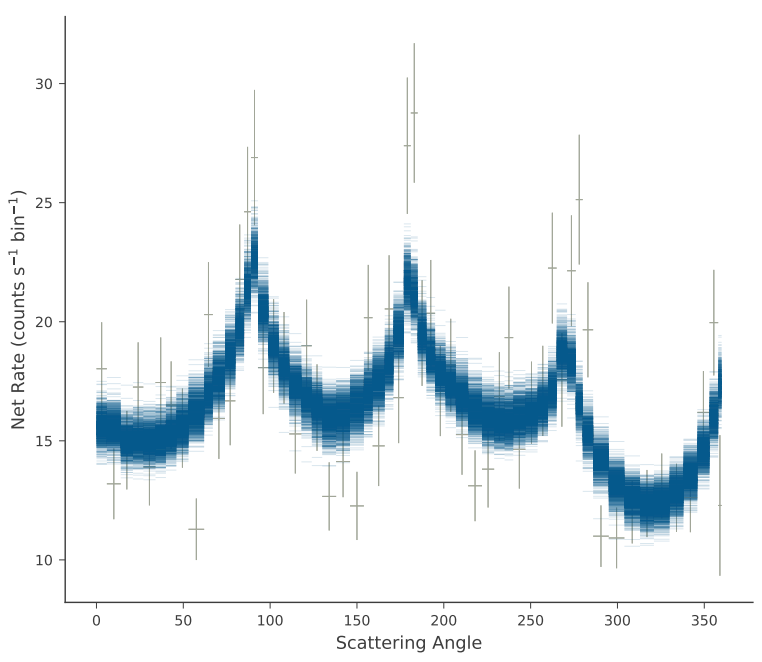

(b)

Fig. I.3. Panel $a$ : polarization posterior distributions for GRB 170206A with the 1 and $2 \sigma$ credibility intervals as well as that corresponding to 99\% credibility. The polarization angle shown here is in the POLAR coordinate system, a rotation in the positive direction of 106 degrees transforms this to the coordinate system as defined by the IAU. Panel $b$ : measured scattering angle distribution (gray data points with a 6 degree bin size) with the posterior model predictions superimposed in blue. The errors on the data points are the Poisson errors corrected for the background. 


\section{Appendix J: 170210A}

The GRB 170210A, a bright, long GRB with many overlapping pulses, was detected by both POLAR and Fermi-GBM. The latter reported a $T 0$ of February 10, 2017 at 02:47:36.58 (UT) (Roberts \& Mailyan 2017), which is taken as $T 0$ for the analysis presented here as well for convenience. A $T_{90}$ of $(47.63 \pm 2.51) \mathrm{s}$ was measured using POLAR data. The light curve using POLAR data, including the signal region (blue) and part of the background region (yellow) can be seen in Fig. J.1. The data from Fermi-GBM was used in this analysis. It should be noted that although some significant emission was seen prior to the main onset of the GRB (at $T 0+30 \mathrm{~s}$ ) these pulses were weak and therefore not taken into account in the analysis here. The spectral fit results showing both the POLAR and Fermi-GBM data can be seen in Fig. J.1. The effective area correction (applied to the POLAR data) found in the analysis is $0.95 \pm 0.02$. The polarization response of POLAR was produced using the location calculated through the IPN (Hurley et al. 2017a): RA $(\mathrm{J} 2000)=226.055^{\circ}, \operatorname{Dec}(\mathrm{J} 2000)=-65.101^{\circ}$, and a localization error of $2^{\circ}$ was assumed in the response. This location implies a significant off-axis incoming angle for the GRB of $80.6^{\circ}$, such an off-axis angle results in a loss of approximately $67 \%$ sensitivity at particular polarization angles (at $45^{\circ}$ and $135^{\circ}$ ) compared to the maximum sensitivity found at $0^{\circ}$ and $90^{\circ}$, as explained in detail in the section regarding GRB 170207A. The posterior distributions of the spectral and polarization parameters are shown in Fig. J.2. Finally, the posterior distribution of the polarization parameters is shown together with measured scattering angle distribution superimposed by samples from the posterior model predictions (blue) in Fig. J.3. The effect of the reduced sensitivity at polarization angles around $135^{\circ}$ can clearly be observed in the posterior distribution. While very constraining measurements are possible at all other angles, the PD is almost fully unconstrained around this angle. A PD of $11.4_{-11.4}^{+35.7 \%}$ is found together with a $99 \%$ upper limit of $95.2 \%$. Although it appears that the posterior distribution is dominated by the lack of sensitivity, the results do clearly exclude high PDs at any polarization angles away from $135^{\circ}$. A time-resolved study was performed, but the polarization results for all studied time bins were found to be similar as for the full GRB.

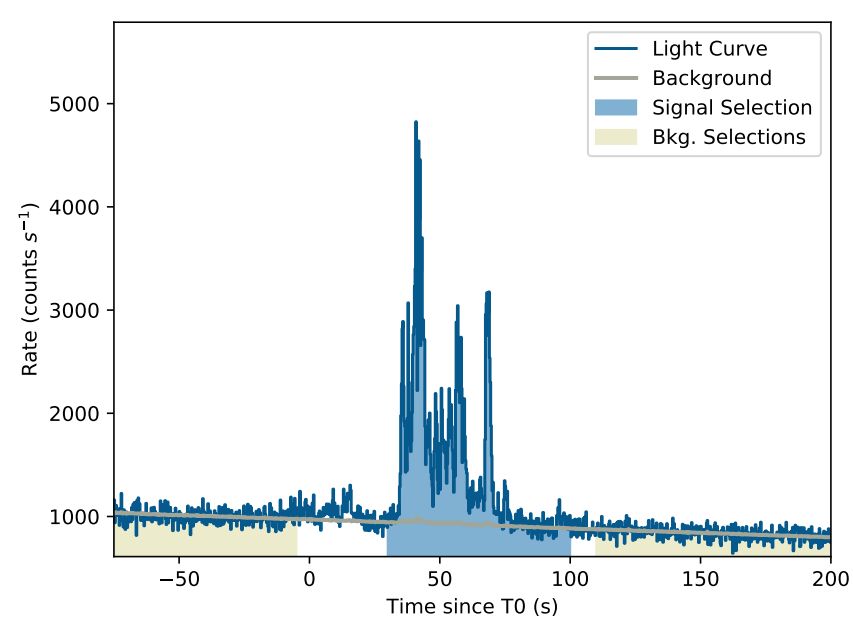

(a)

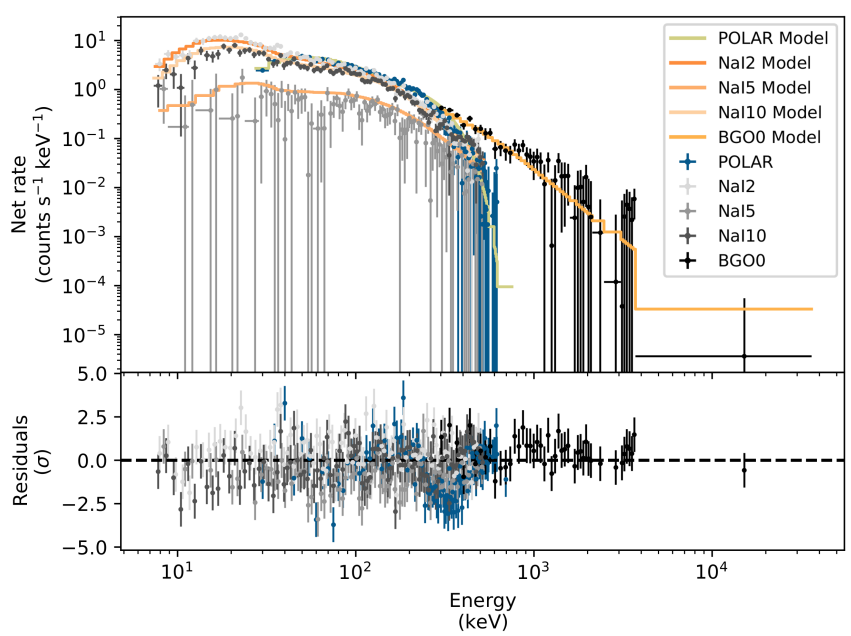

(b)

Fig. J.1. Panel a: light curve of GRB 170210A as measured by POLAR, where $T=0 \mathrm{~s}$ is defined as the $T 0$ employed by Fermi-GBM in their data products for this GRB (Roberts \& Mailyan 2017). Panel $b$ : joint spectral fit result for 170210A. The number of counts as detected by both POLAR (blue) and the different NaI and BGO detectors of FermiGBM (gray tints) are shown along with the best-fitting spectrum folded through the instrument responses in yellow for POLAR data and in orange tints for the Fermi-GBM data. The residuals for both data sets are shown at the bottom of the figure. 

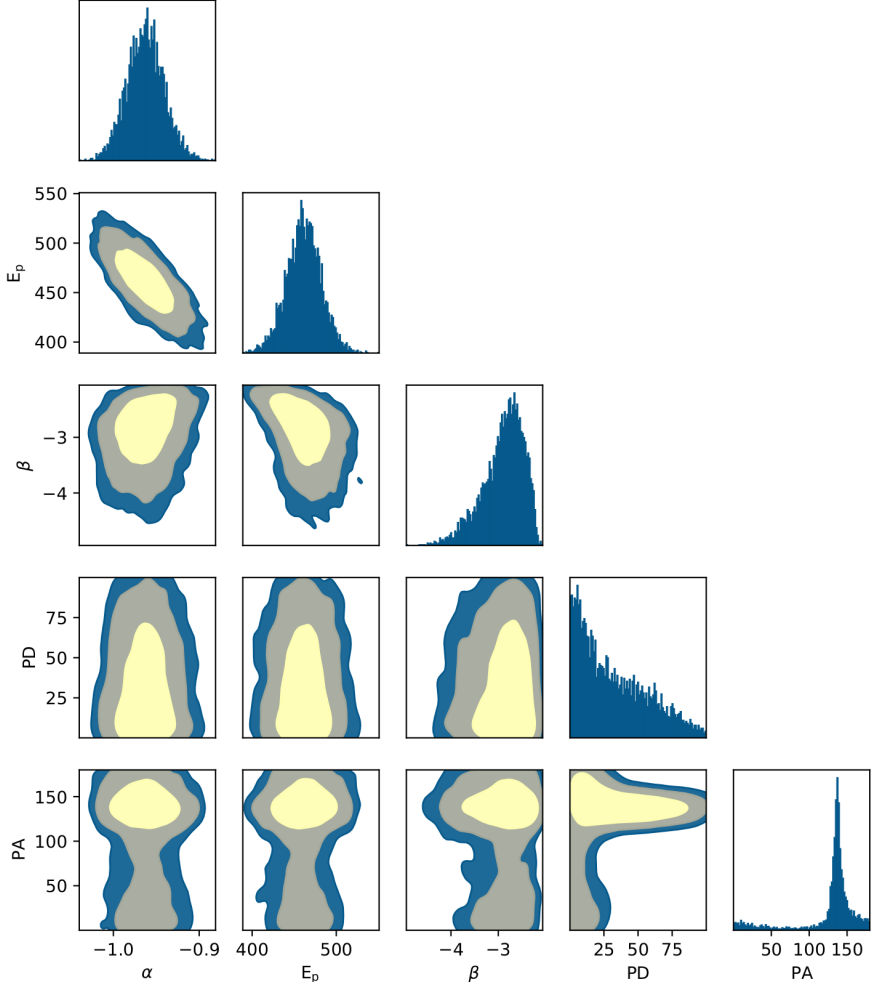

Fig. J.2. Spectral and polarization posterior distributions for GRB $170210 \mathrm{~A}$. The 1 and $2 \sigma$ credibility intervals as well as that corresponding to $99 \%$ are indicated. The polarization angle shown here is in the POLAR coordinate system; a rotation in the positive direction of 55 degrees transforms this to the coordinate system as defined by the IAU.
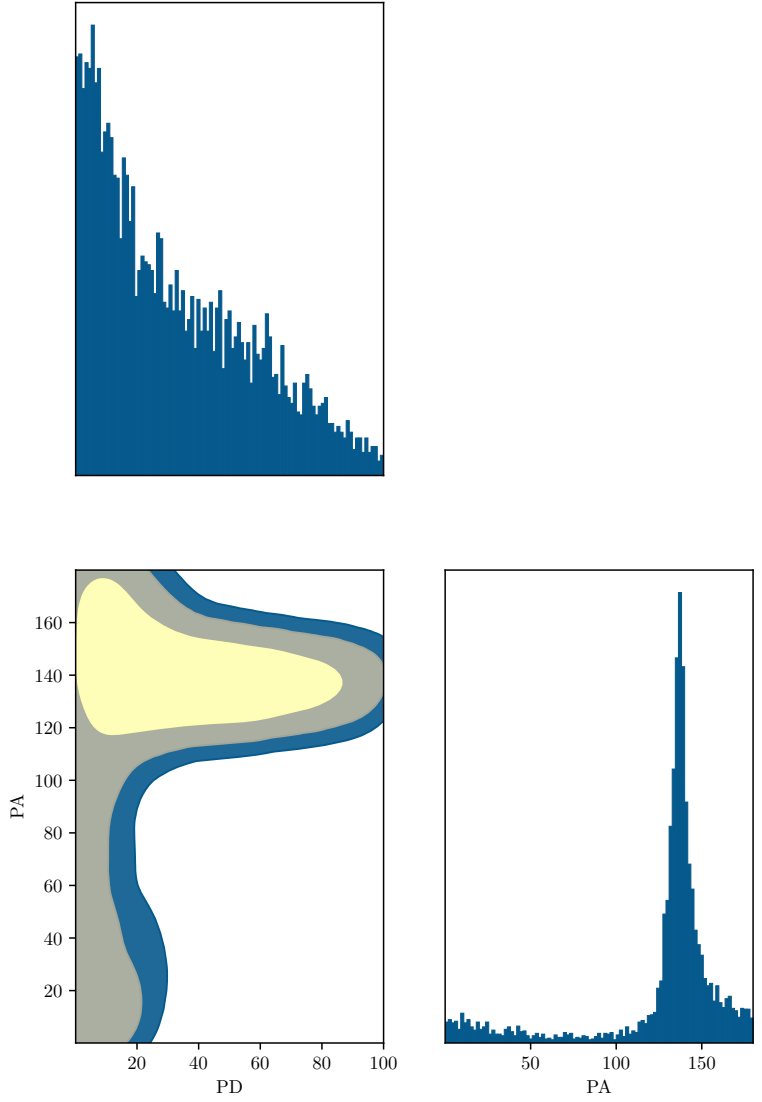

(a)

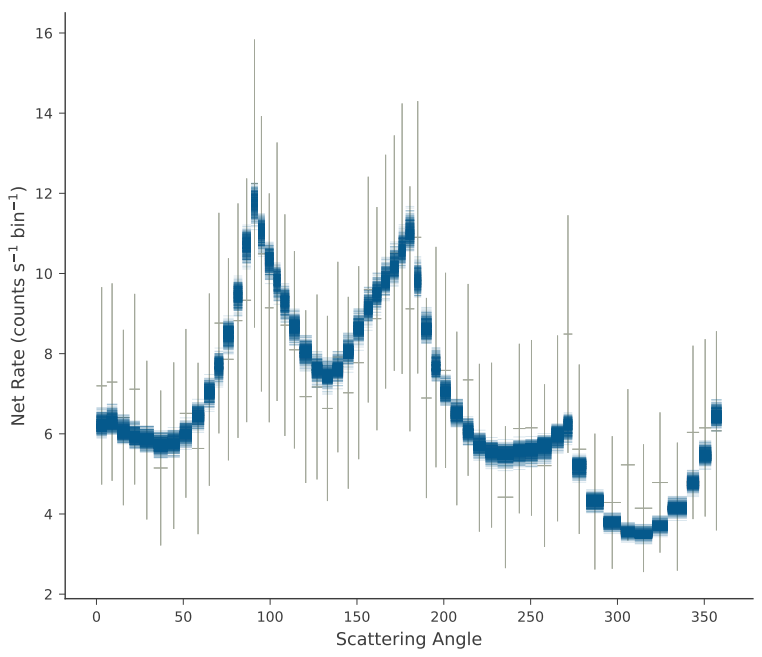

(b)

Fig. J.3. Panel a: polarization posterior distributions for GRB 170210A with the 1 and $2 \sigma$ credibility intervals as well as that corresponding to 99\% credibility. The posterior distribution clearly indicates the lack of sensitivity for this GRB for a polarization angle of $135^{\circ}$. The polarization angle shown here is in the POLAR coordinate system; a rotation in the positive direction of 55 degrees transforms this to the coordinate system as defined by the IAU. Panel $b$ : measured scattering angle distribution (gray data points with a 6 degree bin size) superimposed by samples from the posterior model predictions (blue) is shown. The errors on the data points are the Poisson errors corrected for the background. 


\section{Appendix K: 170305A}

The GRB 170305A, a short GRB, was detected by POLAR and by Fermi-GBM. The latter reported a $T 0$ of March 5, 2017 at 06:09:06.8 (UT) (Stanbro et al. 2017), which is used as the $T 0$ for analysis presented here as well for convenience. A $T_{90}$ of $(0.45 \pm 0.01) \mathrm{s}$ was measured using POLAR data. The light curve, including the signal region (blue) and the background region (yellow) can be seen in Fig. K.1 along with a more detailed inset of the main pulse. Spectral data from Fermi-GBM were used in the joint spectral fit along with the POLAR data. The spectral results of the joint fit can be seen in Fig. K.1. The effective area correction (applied to the POLAR data) found in the analysis is $1.06 \pm 0.07$. The response of POLAR was produced using the location calculated using the IPN (Hurley et al. 2017b): RA $(J 2000)=39.658^{\circ}, \operatorname{Dec}(J 2000)=9.901^{\circ}$, corresponding to an off-axis incoming angle of $31.4^{\circ}$. A localization error of $2^{\circ}$ was assumed in the response. The posterior distributions of the spectral and polarization parameters are shown in Fig. K.2. Finally, the posterior distribution of the polarization parameters is shown together with measured scattering angle distribution superimposed by samples from the posterior model predictions (blue) in Fig. K.3. A PD of $(40.0 \pm 25.0) \%$ is found along with a $99 \%$ credibility upper limit for PD of $97 \%$. The rather large uncertainty can be attributed to the relatively low signal-to-background-counts ratio in the POLAR data for this $\mathrm{GRB}$, which had an average flux of $1.6 \pm 0.2 \times 10^{-6} \mathrm{erg} \mathrm{cm}^{-2} \mathrm{~s}^{-1}$ as measured over one second. This is just above the set lower limit for our cuts.

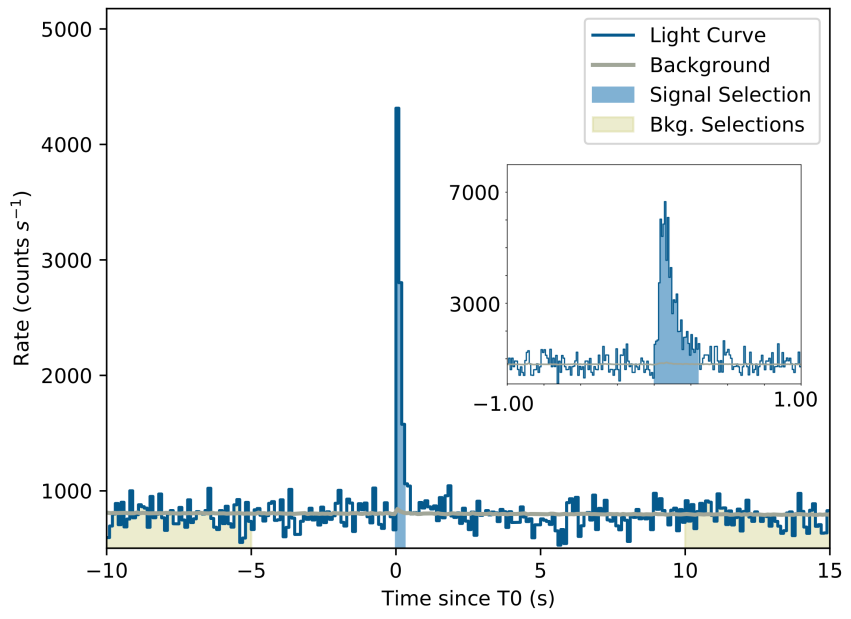

(a)

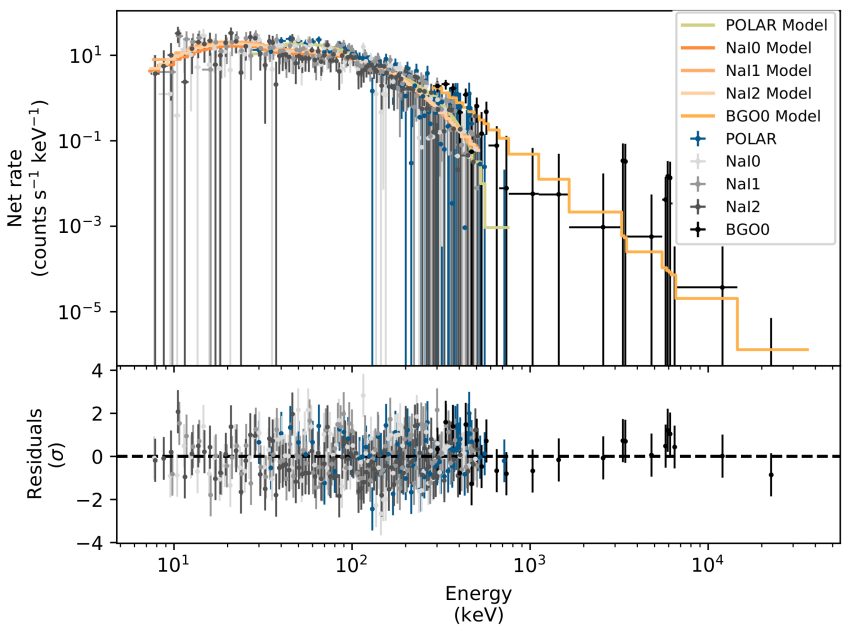

(b)

Fig. K.1. Panel $a$ : light curve of GRB 170305 A as measured by POLAR, with an inlay of a zoomed in version around the peak, where $T=0 \mathrm{~s}$ is defined as the $T 0$ employed by Fermi-GBM in their data products for this GRB (Stanbro et al. 2017). Panel b: joint spectral fit result for 170305A. The number of counts as detected by both POLAR (blue) and the different NaI and BGO detectors of Fermi-GBM (gray tints) are shown along with the best-fitting spectrum folded through the instrument responses in yellow for POLAR data and in orange tints for the Fermi-GBM data. The residuals for both data sets are shown at the bottom of the figure. 

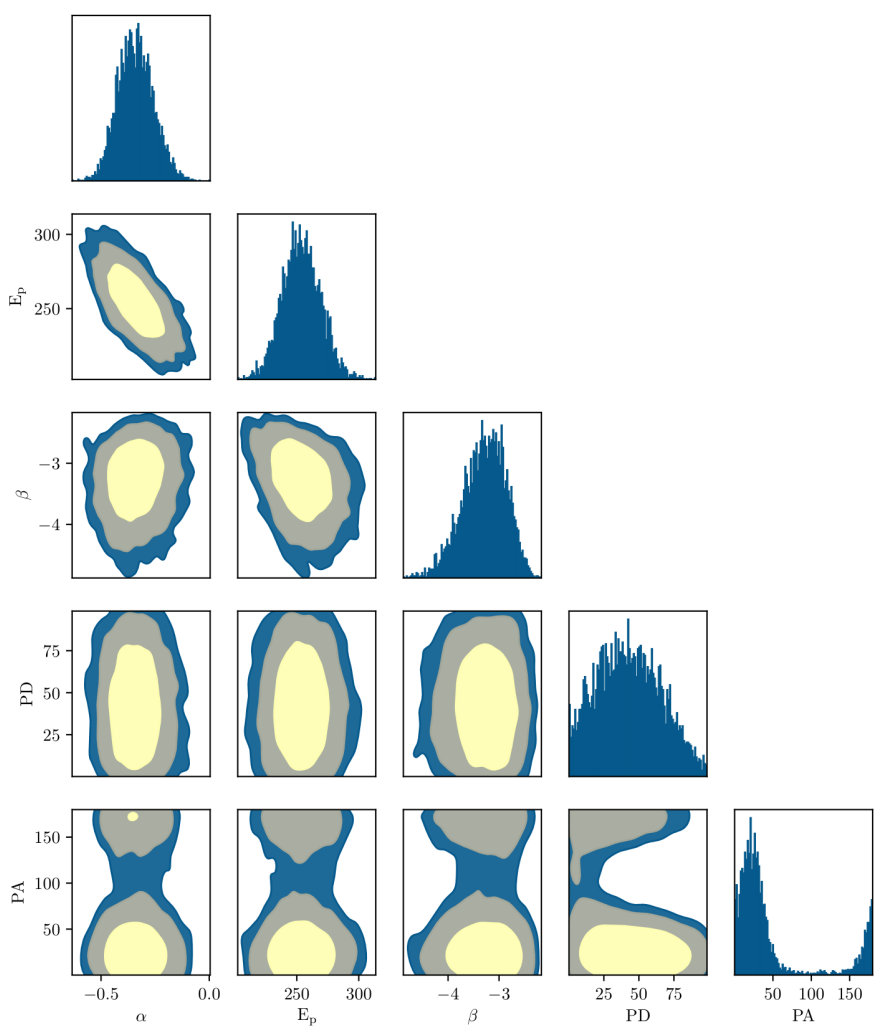

Fig. K.2. Spectral and polarization posterior distributions for GRB 170305A. The $1 \sigma$ and $2 \sigma$ credibility intervals as well as that corresponding to $99 \%$ are indicated. The polarization angle shown here is in the POLAR coordinate system; a rotation in the positive direction of 65 degrees transforms this to the coordinate system as defined by the IAU.
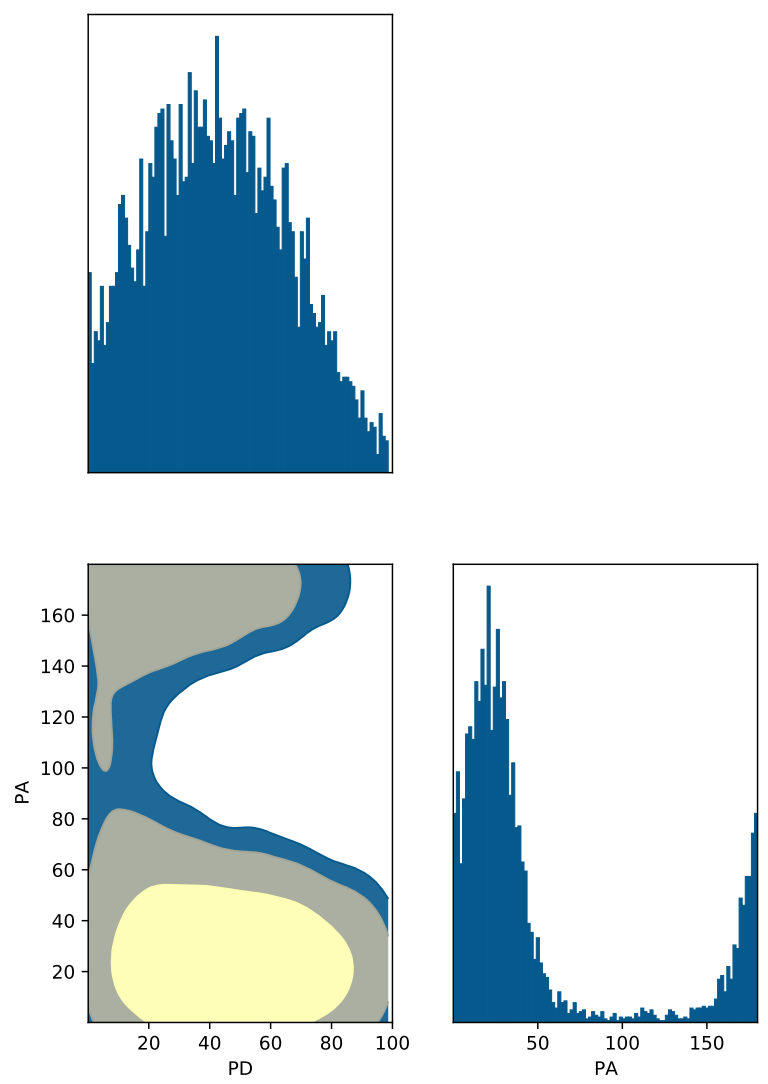

(a)

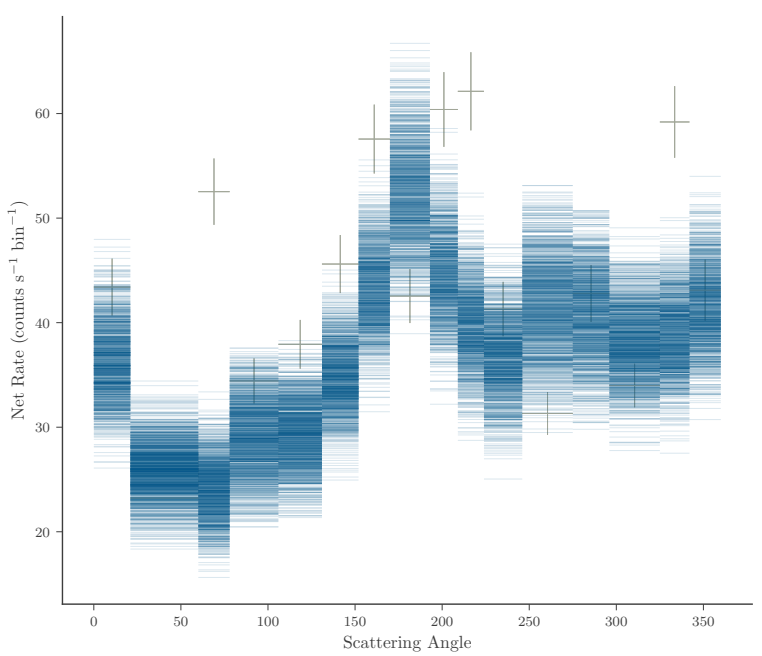

(b)

Fig. K.3. Panel a: polarization posterior distributions for GRB 170305A with the $1 \sigma$ and $2 \sigma$ credibility intervals as well as that corresponding to 99\% credibility. The polarization angle shown here is in the POLAR coordinate system; a rotation in the positive direction of 65 degrees transforms this to the coordinate system as defined by the IAU. Panel $b$ : measured scattering angle distribution (gray data points with a $22.5^{\circ}$ bin size) with samples from the posterior model predictions superimposed in blue. The errors on the data points are the Poisson errors corrected for the background. 


\section{Appendix L: 170320A}

The GRB 170320A was detected by POLAR on March 20, 2017 at 03:42:39.00 (UT) (Zhao et al. 2017) which is defined as $T 0$ for the analysis presented here for convenience. A $T_{90}$ of $(6.83 \pm 0.09) \mathrm{s}$ was measured using POLAR data. The light curve from POLAR data, including the signal region (blue) and part of the background region (yellow) can be seen in Fig. L.1. As it was not detected by Fermi-GBM or Swift-BAT no spectral data from other instruments was available to perform a joint fit. Therefore, the spectral and polarization analysis was performed using the POLAR response only. However, the spectral parameters reported by Konus-Wind in Tsvetkova et al. (2017b) were used as priors for the spectral fit. It should also be noted that while the main emission took place until $T 0+12 \mathrm{~s}$, a soft after-pulse was seen by Konus-Wind (Tsvetkova et al. 2017b), and also seen in the POLAR data around $T 0+30 \mathrm{~s}$. The time period around the after-pulse was excluded from the signal and background selection. The response was produced using the location provided by the IPN (Hurley et al. 2017c): RA $(J 2000)=320.074^{\circ}$, Dec $(\mathrm{J} 2000)=55.060^{\circ}$, where an uncertainty of $1^{\circ}$ on the location was assumed when generating the response. The location corresponds to a large off-axis incoming angle of $84.7^{\circ}$ which reduces the sensitivity for polarization angles of $45^{\circ}$ and $135^{\circ}$ by approximately $85 \%$. The spectral results from the joint fit can be seen in Fig. L.1. The posterior distributions of the spectral and polarization parameters are shown in Fig. 4. Finally, the posterior distribution of the polarization parameters is shown together with measured scattering angle distribution superimposed by samples from the posterior model predictions (blue) in Fig. L.3. A PD of $18.0_{-15.0}^{+28.0} \%$ is found. No significant constraints on the polarization can be provided, which is due to the lack of sensitivity for polarization angles of $45^{\circ}$ and $135^{\circ}$ although we can exclude high values of PD for the majority of polarization angles.

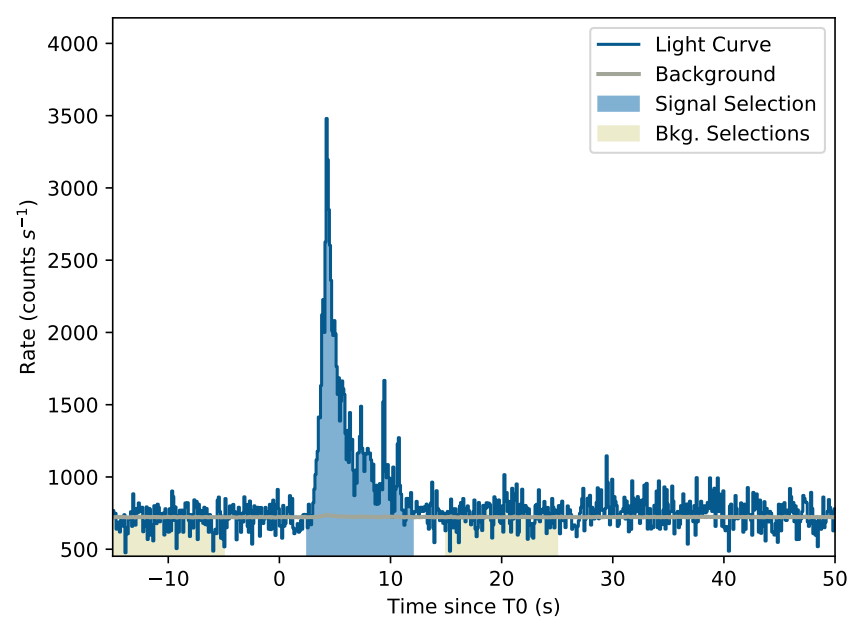

(a)

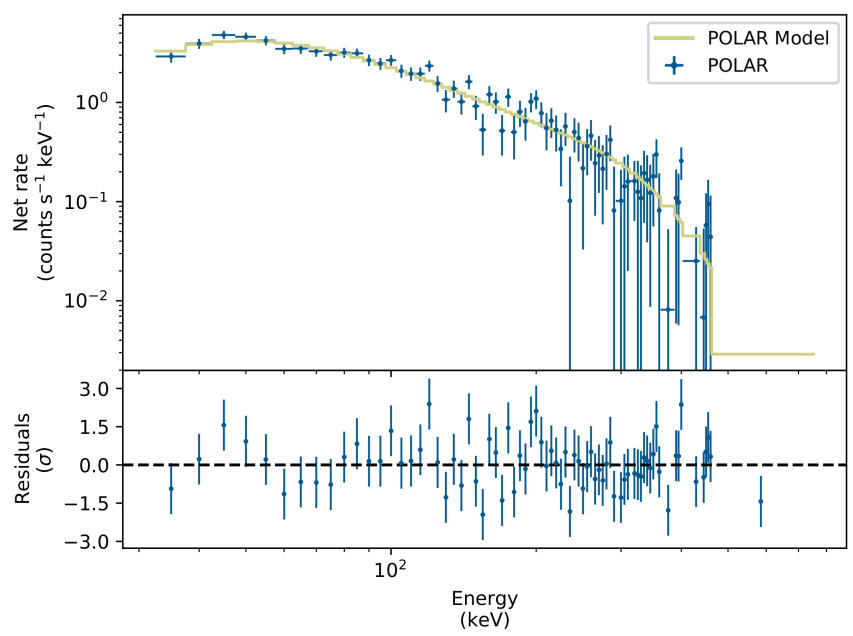

(b)

Fig. L.1. Panel a: light curve of GRB 170320A as measured by POLAR, where $T=0 \mathrm{~s}$ is defined as the $T 0$ reported by POLAR in the original GCN (Zhao et al. 2017). Panel b: joint spectral fit result for 170320A. The number of counts as detected by both POLAR (blue) is shown along with the best-fitting spectrum folded through the instrument response in yellow. The residuals are shown in the bottom of the figure. 

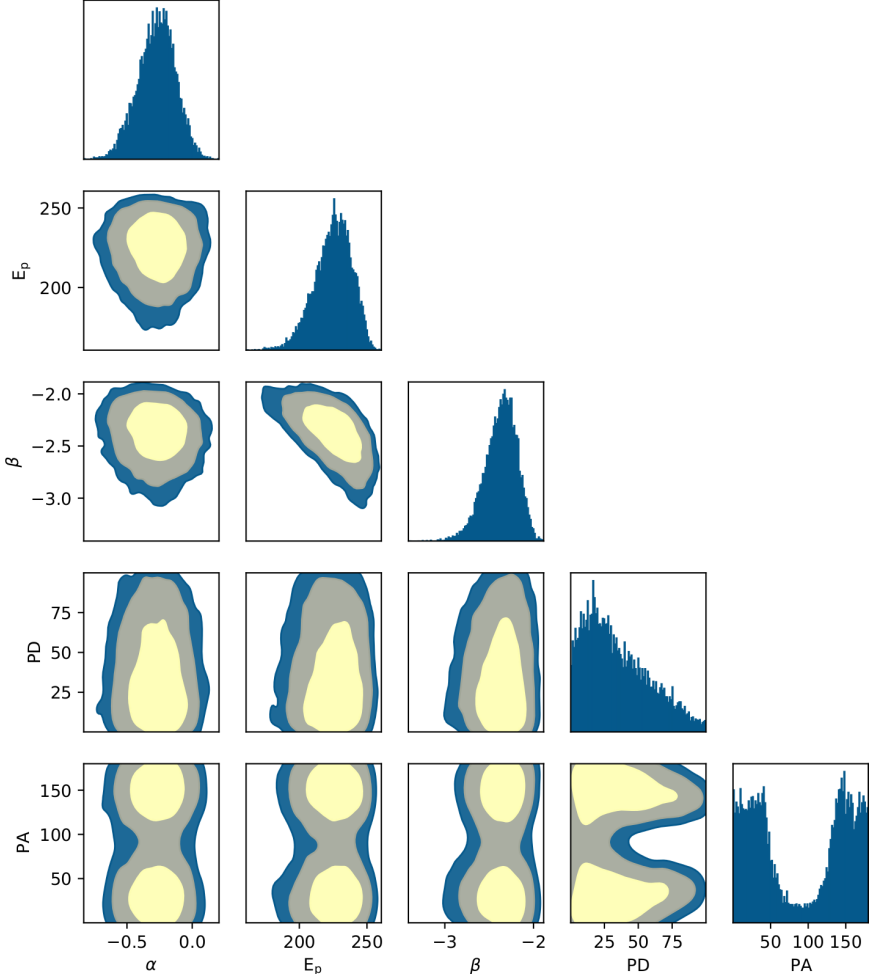

Fig. L.2. Spectral and polarization posterior distributions for GRB 170320A. The $1 \sigma$ and $2 \sigma$ credibility intervals as well as that corresponding to $99 \%$ are indicated. The polarization angle shown here is in the POLAR coordinate system; a rotation in the positive direction of 56 degrees transforms this to the coordinate system as defined by the IAU.
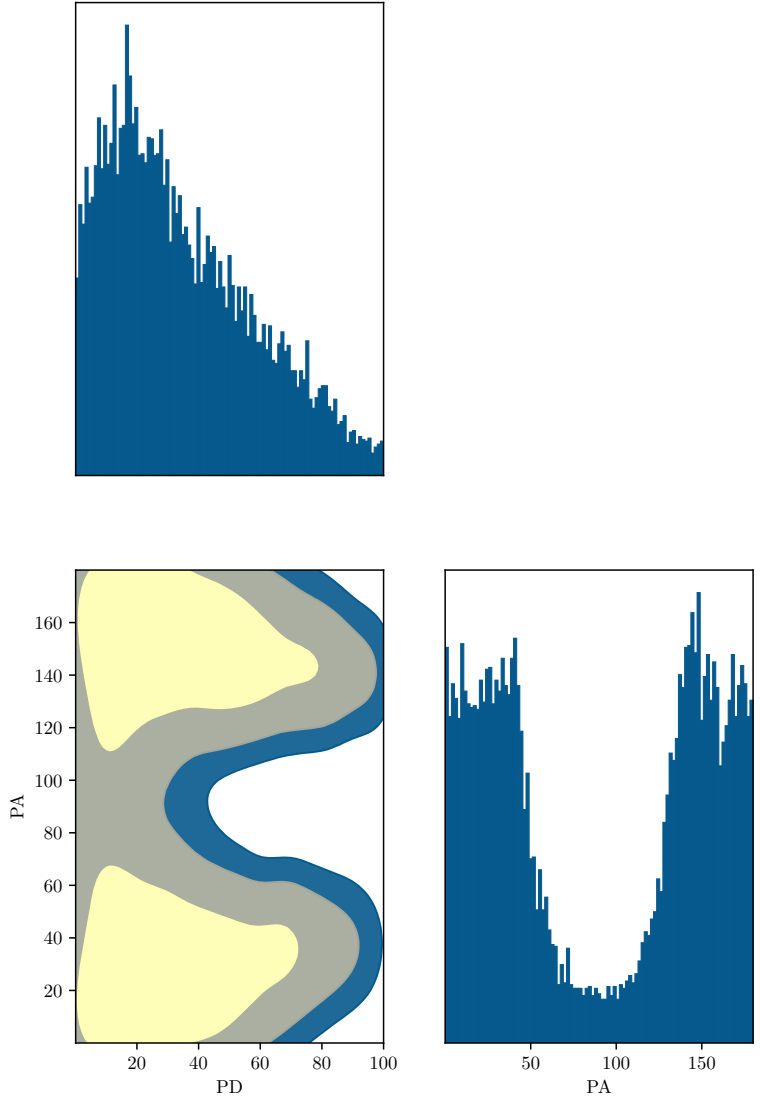

(a)

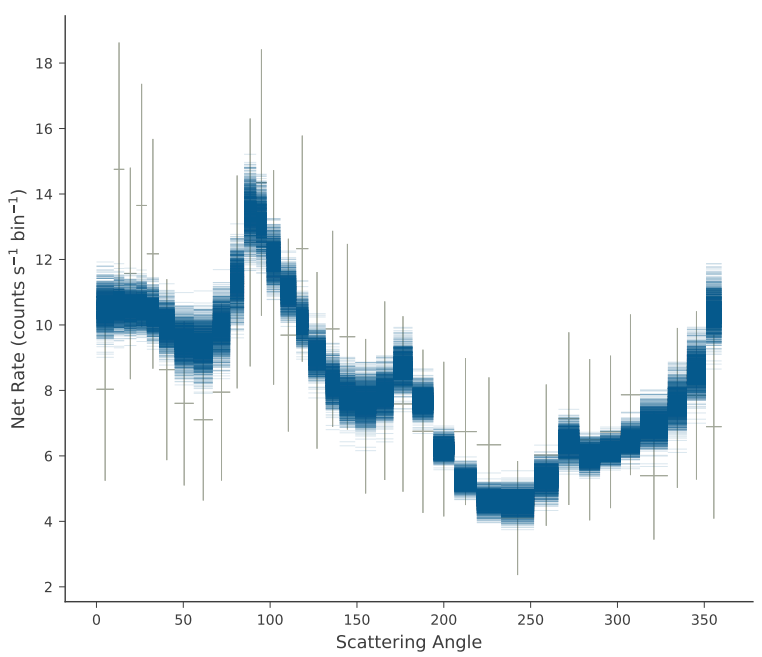

(b)

Fig. L.3. Panel a: polarization posterior distributions for GRB 170320A with the 1 and $2 \sigma$ credibility intervals as well as that corresponding to 99\% credibility. The polarization angle shown here is in the POLAR coordinate system; a rotation in the positive direction of 56 degrees transforms this to the coordinate system as defined by the IAU. Panel $b$ : measured scattering angle distribution (gray data points with a 10 degree bin size) with samples from the posterior model predictions superimposed in blue. The errors on the data points are the Poisson errors corrected for the background. 